L. K. Idriss et al., Static behaviour of different types of R.C beam-column connections as affected by both value acting lateral horizontal force and grade of used concrete (theoretical study) part two, pp.746 - 814

\title{
STATIC BEHAVIOUR OF DIFFERENT TYPES OF R.C BEAM- COLUMN CONNECTIONS AS AFFECTED BY BOTH VALUE ACTING LATERAL HORIZONTAL FORCEAND GRADE OF USED CONCRETE (THEORETICAL STUDY) Part Two
}

\author{
A. M. Ahmed ${ }^{1}$, M. M. Rashwan ${ }^{2}$, L. K. Idriss ${ }^{3 \text {, * }}$ \\ Civil Engineering Department, Faculty of Engineering, Assiut University
}

Received 11 November 2012, accepted 26 December 2012

\begin{abstract}
This paper describes a theoretical study of forty eight (48) Reinforced Concrete RC beam column joints, which generally classified as interior, exterior and corner joints. The most main parameters, which may influence the behavior of beam-column joint, transverse reinforcement, lateral shear reinforcement, longitudinal reinforcement, joint area, column axial load, concrete compressive strength, end boundary conditions ...etc. In this research FEA (finite element analysis) using ABAQUSICAE commercial software will be developed to build a detailed model to predict the nonlinear behavior of beam-column joint under seismic loadings. The ABAQUSICAE 6.7 solution is a practical way to implement the nonlinear dynamic earthquake analysis for the finite element model. Our study is focusing with joints where statically lateral load was applied and gradually increased maintaining constant axial load at the top of the column equal to $0.15\left(\mathrm{~A}_{\mathrm{c}} \mathrm{F}_{\mathrm{c}}\right)$. Several linear variable horizontal displacement transducers were mounted, the net story drift, bond stress, axial shear stresses and strains as well as energy absorption for using different grades of concrete C250, C400, C600 and C1200 are evaluated and discussed to illustrate the behaviour of the studied joints under the case study of loadings.
\end{abstract}

Keywords: RC Beam-Column Connections, Concrete Compressive Strength, Lateral Load, Shear and Bond Strength, Joint Deformation, Energy Absorption, Mode of Failure, and ABAQUSICAE.

\section{Introduction}

There are several parameters, which may influence the behavior of beam-column joint. Among of these parameters are the followings:

1. Type of joint: which is mainly referred as interior joint, exterior joints or corner joints.

2. Transverse Reinforcement, an increase of joint transverse reinforcement causes an increase of joint shear stiffness. Because the capacity of both diagonal concrete strut and truss mechanisms is dependent on concrete compressive strength (Jaehong K. et al. 2008). Also(Ahmed H. 2003) summarized that the increase in transverse reinforcement above certain amount had a little effect on the ultimate strength of the joint and the transverse reinforcement in the joint is more effective in maintaining the strength than the stiffness.

3. Lateral Shear Reinforcement, The amount of joint lateral reinforcement has a significant effect on both strangled deformation capacity of the joints (Kazuhiro K. (1991).

\footnotetext{
* Corresponding author.

Civil Engineering Department, Faculty of Engineering, Assiut University
} 
L. K. Idriss et al., Static behaviour of different types of R.C beam-column connections as affected by both value acting lateral horizontal force and grade of used concrete (theoretical study) part two, pp.746 - 814

4. Longitudinal Reinforcement, Hitoshi S.et al., (2002) studied the resultants in longitudinal bar do not exceeds the yield strength of the reinforcement, and the bars contributed only on the axial load carrying capacity .

5. Joint Area, S. R. Uma et al., (2006) study proved that the effective, joint area $A_{j}$ is the area resisting the shear within the joint and is contributed by the framing members in the considered direction of loading.

6. Column Axial Load Kumar et al., (2002) found that the joint rotation and the axial load in the column increase the ductility and energy dissipation capacity and reduced the joint region damage.

7. Concrete Compressive Strength, Jaehong K. et al., (2008); summarized that concrete compressive strength is the most important parameter in determining RC joint shear, (Laura N. Lowes et al. 2004); indicated that concrete compressive strength is substantially less than that observed under monotonic loading and that concrete tensile strength deteriorates more rapidly as a function of tensile strain.

8. Miscellaneous factors: including lateral loads ratios of columns connected beams dimensions , boundary conditions .....etc., which are not available for the authors.

9. The following is a brief for the available main items and concept concerning and defining the characteristic of the behaviour of R.C joints:

10. Shear Strength, Codes Recommendations for Joint Shear Strength:

1. ACI 352R-95 and ACI 318-02

For modern RC beam-column connections, American Concrete Institute (maintaining proper confinement within a joint panel), has defined a nominal joint shear strength; that is: as Eq. (1).

$$
V_{\mathrm{n}}=\gamma_{\mathrm{ACl}} \sqrt{f_{\mathrm{c}}^{\prime}} \mathrm{b}_{\mathrm{j}} \mathrm{h}_{\mathrm{c}}
$$

Where: $\left(\gamma_{\mathrm{ACI}}\right)$ is the joint shear strength factor, $f_{\mathrm{c}}$ is the specified concrete compressive strength, $\left(b_{j}\right)$ is the effective joint shear width, and $\left(h_{c}\right)$ is the column depth.

2. ECCS 203 (2001)

Egyptian code for concrete structure sets the nominal shear strength of the joint $\left(\tau_{\mathrm{J}}\right)$ as a function of concrete strength only, $\left(\mathrm{A}_{\mathrm{j}}\right)$ is the effective joint area, $\left(f_{\mathrm{c}}^{\prime}\right)$; the cylinder compressive strength of concrete is given as Eq. (2);

$$
\tau_{\mathrm{j}}=0.96 A_{\mathrm{j}} \sqrt{f_{\mathrm{c}}^{\prime}}
$$

3. AIJ 1997

Architectural Institute of Japan "Design guidelines for earthquake resistant reinforced concrete building based on ultimate strength concept and commentary" has recommended a nominal joint shear strength in the form of Eq. (3); that is:

$$
\mathrm{V}_{\mathrm{j}}=\mathrm{k} \phi f_{\mathrm{j}} \mathrm{b}_{\mathrm{j}} \mathrm{D}_{\mathrm{j}}
$$

where, $\mathrm{k}$ is the factor dependent on the shape of in-plane geometry $(=1.0$ for interior connections, $=0.7$ for both exterior connection,$T$-shape top story joints and $=0.4$ for corner knee connections); $(\phi)$ is the factor for the effect of out-of-plane geometry (1.0 for joints with transverse beams on both sides and 0.85 for other types of joints); $\left(f_{j}\right)$ is the standard value of the joint shear strength (as a function of concrete compressive strength); $\left(b_{j}\right)$ is the effective joint shear width; and $\left(D_{\mathrm{j}}\right)$ is the effective column depth.

Journal of Engineering Sciences, Assiut University, Faculty of Engineering, Vol. 41, No. 3, May, 2013,E-mail address: jes@aun.edu.eg 
L. K. Idriss et al., Static behaviour of different types of R.C beam-column connections as affected by both value acting lateral horizontal force and grade of used concrete (theoretical study) part two, pp.746 - 814 .

The standard value of the joint shear strength $\left(f_{\mathrm{j}}\right)$ is suggested as given by equation (4); that is:

4. NZS 3101: 1995

$$
f_{\mathrm{j}}=0.8 \times\left(f_{\mathrm{c}}^{\prime}\right)^{0.7}
$$

Standards New Zealand (Concrete Structures Standard) has suggested the design joint shear strength as Eq. (5) that is:

$$
\mathrm{V}_{\mathrm{j}}=\mathrm{v}_{\mathrm{j}} \mathrm{b}_{\mathrm{j}} \mathrm{h}_{\mathrm{c}}
$$

where $\left(v_{j}\right)$ is the joint shear stress, $\left(b_{j}\right)$ is the effective joint shear width, and $\left(h_{c}\right)$ is the column depth. Joint shear stress is defined as Eq. (6) that is:

$$
V_{\mathrm{j}}=\frac{f_{\mathrm{c}}}{6 \alpha} \frac{f_{\mathrm{jy}} A_{\mathrm{jh}}}{f_{\text {by }} A_{\mathrm{s}}^{*}}
$$

In Eq. (6) $(\alpha)$ : is the parameter considering column axial load; $\left(f_{\mathrm{jy}}\right)$ is the yield stress of horizontal joint transverse reinforcement; $\left(\mathrm{A}_{\mathrm{jh}}\right)$ is the total cross-sectional area of horizontal joint transverse reinforcement; $\left(f_{\text {by }}\right)$ is the yield stress of longitudinal beam reinforcement; and $\left(A_{s}^{*}\right)$ is the greater of the area of top or bottom beam reinforcement passing through the joint (excluding bars in an effective tension flange).

5. EN 1998-1:2003

Euro code has limited the nominal shear stress, $\left(v_{\mathrm{jh}}\right)$ within interior beam column joint to be less than the stress value given by the Eq. (7).

$$
V_{\mathrm{jh}} \leq \eta f_{\mathrm{cd}} \sqrt{1-\frac{V_{\mathrm{d}}}{\eta}}
$$

where $\eta=0.6\left(1-\frac{\mathrm{f}_{\mathrm{c}^{\prime}}^{\prime}}{250}\right)$ enotes the reduction factor on concrete compressive strength due to tensile strains in transverse direction.

6. AIJ Guidelines derived from Japanese database of the tests of beam-column joint without transverse beams. They are given by the equations,

$$
\begin{array}{ll}
\tau_{j u}=1.56 \times \sigma_{B}^{0.712} & \text { for interior beam-column joint } \\
\tau_{j u}=1.13 \times \sigma_{B}^{0.718} & \text { for exterior beam-column joint }
\end{array}
$$

where, $\left(\tau_{j u}\right)$ is joint shear strength and $\left(\sigma_{B}\right)$ is concrete compressive strength.

7. Bond Strength:

A maximum bond stress $\mathrm{Bu}$ of beam reinforcement over the column width was estimated by assuming simultaneous yielding of the beam reinforcement in tension and compression at the two faces of the joint. Bond-strength values, required to complete calibration of the model, are defined on the basis of experimental data provided by a number of different researchers. The results of previous research indicate that bond strength is a function of the material state of the anchored bar as well as of the concrete and transverse reinforcing steel in the vicinity of the reinforcing bar (Lowes, L. 2002). Bond strength is relatively high if reinforcement is anchored in a reinforced concrete zone that carries compression perpendicular to the bar axis, and relatively low if the reinforced concrete carries tension (Eligehausen, et al., 1993). Further, bond strength

Journal of Engineering Sciences, Assiut University, Faculty of Engineering, Vol. 41, No. 3, May, 2013,E-mail address: jes@aun.edu.eg 
L. K. Idriss et al., Static behaviour of different types of R.C beam-column connections as affected by both value acting lateral horizontal force and grade of used concrete (theoretical study) part two, pp.746 - 814

is reduced for reinforcement carrying stress in excess of the tensile yield strength and increased for reinforcement carrying compressive stress less than the compressive yield strength (Shima et al., 1997). The bond strength was assumed proportional to the square root of the concrete compressive. Laura, N. (1992).

8. Flexure Strength, Deformation as shear deformation of the beam-column connection increases, while the remaining shear-resisting capacity of the connection is reserved (Hitoshi Sh. 2004).

9. Energy Absorption and Effect of End Boundary Conditions the sub assemblages were tested by (John S. et al., 2001) within a reaction frame with loading and boundary conditions Pin connections were attached to the sub assemblages at approximate locations of points of contra flexure under lateral loading.

10. Failure Modes, There are Three Failure Modes for Beam-Column Joints: A) The new behavior model for shear failure of an interior beam-column joint is illustrated by (Hitoshi Sh. 2004) B) The analytical deformed shape and cracking pattern of specimen JL are compared with the experimental results by (Teeraphot Su. et al., 2008). The FEM demonstrates an extensive deformation at the ends of beam framing into the joints, indicating a flexural failure, C) Beam bending failure with a ductile load-deflection behavior model well illustrated by (Josef H., et al., 2003) The ultimate bending moment capacity of the beam is reached and the beam reinforcement is yielding, and joint failure in which the ultimate bending moment capacity of the beam cannot be reached and a characteristic crack is observed inside the joint. D) Extensive cracking within the joint core region can be found in the early stages, and the cracks open widely is the horizontal displacement increases (Bing Li, et al., 2003).

To investigate and compare the behaviors of the joints of the following detailed dimensions and end conditions e (48)joint, the normal-strength and high-strength joints are both representative of an interior, exterior and corner joint are chosen. Different lateral loads were applied maintaining constant axial load at the top of the column equal to $0.15\left(\mathrm{~A}_{\mathrm{c}} \mathrm{F}_{\mathrm{c}}\right)$, to measure and evaluated the net story drift, shear deformations, axial, shear stresses, bond stress, and total absorbed energy for such joints having different grades of concrete C250,C400,C600 and C1200.

\section{Details of R.C joints}

Table1 and Fig.1 include the connection between the main beam, column, slab. (48) Joints, which taken into account.

Beam sizes for all joints of $\left(b_{b} \times d_{b}\right) \mathrm{mm}(250 \mathrm{~mm}$ wide by $300 \mathrm{~mm}$ deep) $\mathrm{mm}$ as well as column sizes of $\left(b_{c} \times d_{c}\right) \mathrm{mm}(300 \times 300 \mathrm{~mm})$, were kept constant.

The total height of the columns was the same for both specimens at $\mathrm{H}=2.0 \mathrm{~m}$, which gave $\mathrm{L} / 2=1.5 \mathrm{~m}$ above and below the beam. The floor slabs for both specimens were of equal sizes, with a thickness of $120 \mathrm{~mm}$. The boundary conditions, beam ends were supported by horizontal rollers, while the bottom of the column was supported by a constant mechanical hinge too.

Journal of Engineering Sciences, Assiut University, Faculty of Engineering, Vol. 41, No. 3, May, 2013,E-mail address: jes@aun.edu.eg 
L. K. Idriss et al., Static behaviour of different types of R.C beam-column connections as affected by both value acting lateral horizontal force and grade of used concrete (theoretical study) part two, pp.746 - 814 .

All the specimens were cast in concrete of specified characteristic cube strength $\left(f_{\mathrm{c}}^{\prime}\right)$, of 250-400-600 and $1200 \mathrm{~kg} / \mathrm{cm}^{2}$, with yield stress for reinforcing bar $\left(f_{y}\right)$ 2400-28003600 and $4000 \mathrm{~kg} / \mathrm{cm}^{2}$ respectively.

The reinforcement details of all the specimens were identical in Figures 1.a for interior joint, 1.b for exterior joint and 1.c for corner joint, apart from the joints. The beam was equally reinforced at the top and bottom by four high-yield deformed bars of $16 \mathrm{~mm}$ diameter $\left(A_{b s}\right)$ (i.e. $\left.4 \Phi 16,4 \Phi 16\right)$.

Stirrups bent $\left(\rho_{w}\right)$ from 6-mm-diameter mild steel round bars with specified characteristic yield strength of $2400 \mathrm{~kg} / \mathrm{cm}^{2}$ were provided at 80 mm centers (i.e. $\Phi 6 @ 80$ stirrups). The column contained $\left(A_{c s}\right) 12 \Phi 16$ longitudinal reinforcing bars evenly distributed around the perimeter. The transverse reinforcement in the column comprised $\Phi 6$ square hoops and R6 cross ties in two perpendicular directions at $80 \mathrm{~mm}$ centers, as shown in Table 1.

\section{Mesh arrangement}

The mesh module allows you to generate meshes at Figures 2.a, 2.b, 2.c, on assemblies created with ABAQUS\CAE various levels of automation and control are available so that you can create a mesh that meets the needs of your analysis. Mesh refinement is required. When severely nonlinear material Models are used, however, increasing the number of element can increasing constrains within the model. These reduce shear deformation that can lead to an overlay stiff load deflection response

\section{(Abaqus. 2000).}

\section{Boundary conditions and applied loading}

The loading set up is shown in Figures 3.a, 3.b, 3.c for interior and exterior and knee joint. The specimens were supported in vertical position. The top of the column was loaded by two actuators in vertical and horizontal directions. The beam ends were supported by horizontal rollers, while the bottom of the column was supported by a mechanical hinge. The distance between two loading points for beams and columns were $3,000 \mathrm{~mm}$ and $2,000 \mathrm{~mm}$ respectively. With different lateral load was applied maintaining constant axial load at the top of the column equal to $0.15 \mathrm{AcFc}$. The lateral load applied was gradually increased. Several linear variable displacement transducers were mounted on the test specimens to measure the net story drift, bond stress, shear deformations, axial, shear stresses strains, and energy absorption.

The modifications were based on nonlinearity analytically model carried out by (Sam Lee 2008) The degradation factors for compression (dc) and tension (dt) are dependent on the plastic strain (ABAQUS, v6.5).

The nonlinearity of the structure includes geometry nonlinearity, material nonlinearity and a combination of both.

Journal of Engineering Sciences, Assiut University, Faculty of Engineering, Vol. 41, No. 3, May, 2013,E-mail address: jes@aun.edu.eg 
L. K. Idriss et al., Static behaviour of different types of R.C beam-column connections as affected by both value acting lateral horizontal force and grade of used concrete (theoretical study) part two, pp.746 - 814

\section{Geometry nonlinearity}

Geometry nonlinearity can be modeled accurately by using of the Green strain formula. The P- $\square$ effects and large deflection effects are automatically taken into account. Most general finite element analysis packages have this built-in function available

Table 1.

Properties of Joints.

\begin{tabular}{|c|c|c|c|}
\hline $\begin{array}{c}\text { Type of beam-column } \\
\text { joints }\end{array}$ & Interior joint & $\left.\right|_{\text {Exterior joint }}$ & Corner joints \\
\hline (a) Beam $\left(b_{b} \times d_{b}\right) \mathrm{mm}$ & $(250 \times 300)$ & $(250 \times 300)$ & $(250 \times 300)$ \\
\hline Top bars & $4 \Phi 16$ & $4 \Phi 16$ & $4 \Phi 16$ \\
\hline $\mathrm{a}_{\mathrm{t}} \mathrm{mm}^{2}$ & 804 & 804 & 804 \\
\hline $\mathrm{p}_{\mathrm{t}} \%$ & 1.18 & 1.18 & 1.18 \\
\hline Bot. bars & $4 \Phi 16$ & $4 \Phi 16$ & $4 \Phi 16$ \\
\hline $\mathrm{a}_{\mathrm{t}} \mathrm{mm}^{2}$ & 804 & 804 & 804 \\
\hline $\mathrm{p}_{\mathrm{t}} \%$ & 1.18 & 1.18 & 1.18 \\
\hline Stirrups & 2- $\Phi 6$ & $2-\Phi 6$ & $2-\Phi 6$ \\
\hline @ (mm) & 80 & 80 & 80 \\
\hline $\mathrm{P}_{\mathrm{w}} \%$ & 0.64 & 0.64 & 0.64 \\
\hline (b) column $\left(b_{c} \times d_{c}\right) \mathrm{mm}$ & $(300 \times 300)$ & $(300 \times 300)$ & $(300 \times 300)$ \\
\hline Total Bars & $12-\Phi 16$ & $12-\Phi 16$ & $12-\Phi 16$ \\
\hline$a_{g} m^{2}$ & 2412 & 2412 & 2412 \\
\hline $\mathrm{p} g_{\mathrm{g}} \%$ & 2.62 & 2.62 & 2.62 \\
\hline Hoops & $2 \Phi \mathrm{D} 6$ & 2 ФD6 & 2 ФD6 \\
\hline @ (mm) & 80 & 80 & 80 \\
\hline $\mathrm{P}_{\mathrm{w}} \%$ & 0.27 & 0.27 & 0.27 \\
\hline (c) connection $\mathrm{H} \times \mathrm{L} \mathrm{mm}$ & $(2000 \times 3000)$ & $(2000 \times 1500)$ & $(1000 \times 1500)$ \\
\hline Hoops & 3- $\Phi 6$ & 3- $\Phi 6$ & 3- $\Phi 6$ \\
\hline Sets & $3 @ 60$ & $3 @ 60$ & $3 @ 60$ \\
\hline$a_{w} m^{2}$ & 192 & 192 & 192 \\
\hline $\mathrm{p}_{\mathrm{w}} \%$ & 0.38 & 0.38 & 0.38 \\
\hline Shape & Closed & Closed & Closed \\
\hline (d) Slabs thick. $\mathrm{t}_{\mathrm{s}} \mathrm{cm}$ & 12 & 12 & 12 \\
\hline Longitudinal Dir & $24 \mathrm{D} 8$ & $24 \mathrm{D} 8$ & $24 \mathrm{D} 8$ \\
\hline @ (mm) & 100 & 100 & 100 \\
\hline Stirrups ratio \% & 0.38 & 0.38 & 0.38 \\
\hline Transverse Dir. & $24 \mathrm{D} 8$ & $24 \mathrm{D} 8$ & $24 \mathrm{D} 8$ \\
\hline$@$ (mm) & 200 & 200 & 200 \\
\hline Stirrups ratio \% & 0.27 & 0.27 & 0.27 \\
\hline
\end{tabular}

Note: $a_{t}:$ total area of tensile reinforcement, $p_{t}:$ tensile reinforcement ratio, $a_{g}:$ total area of longitudinal reinforcement, $\mathrm{p}_{\mathrm{g}}$ : gross reinforcement ratio, aw : total area of web reinforcement placed between top and bottom beam bars, $\rho_{\mathrm{w}}:$ web reinforcement ratio, $\mathrm{Ec}=14000 \sqrt{ } f^{\prime} \mathrm{c} \mathrm{kg} / \mathrm{cm}^{2}, \mathrm{Es}=2.2 \times 10^{6} \mathrm{~kg} / \mathrm{cm}^{2}, f_{\mathrm{y}}$ : yield strength, $f^{\prime}$ : concrete compressive strength, $f_{\mathrm{t}}^{\prime}$ : concrete tensile strength.

Journal of Engineering Sciences, Assiut University, Faculty of Engineering, Vol. 41, No. 3, May, 2013,E-mail address: jes@aun.edu.eg 


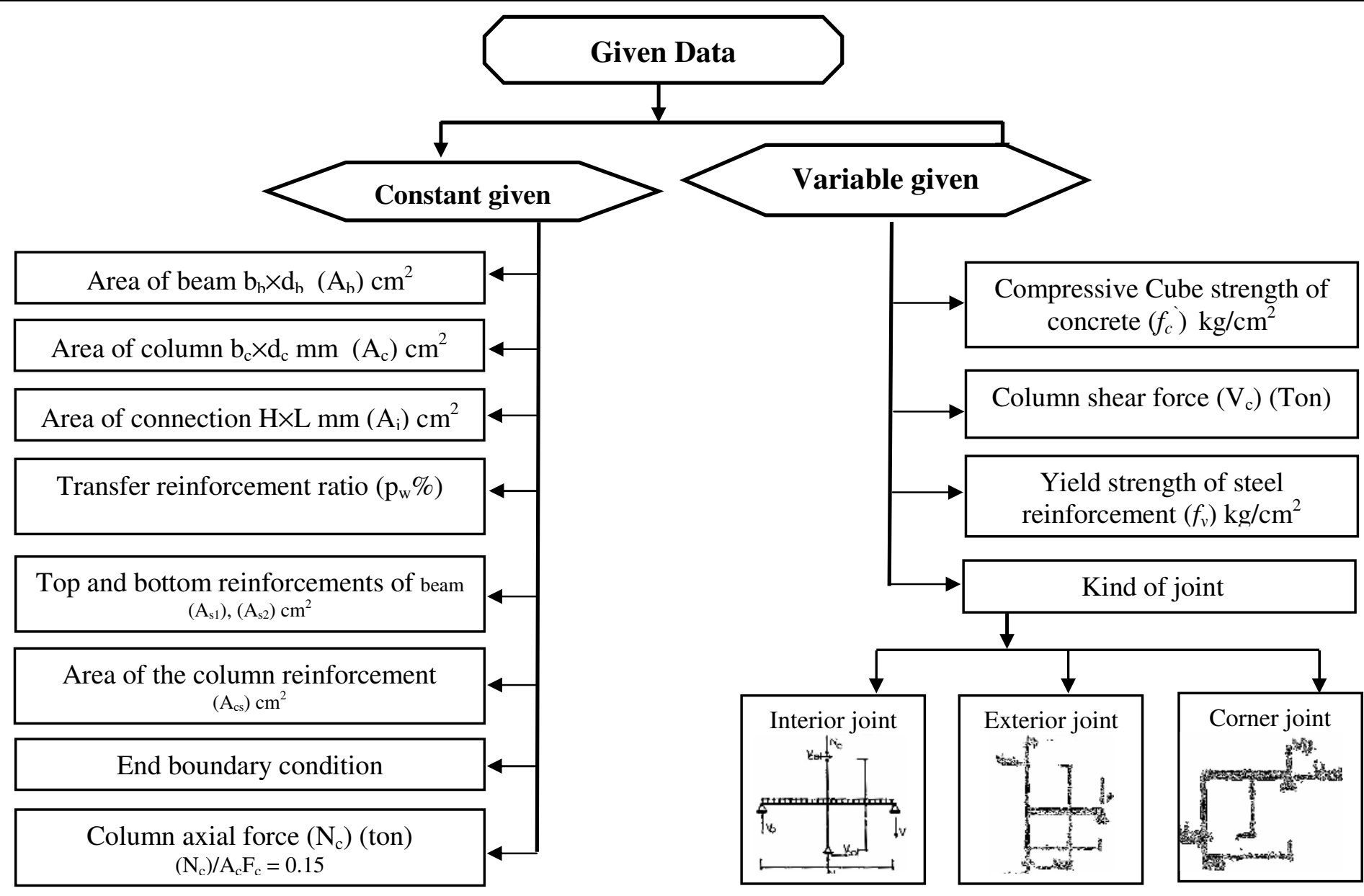

Journal of Engineering Sciences, Assiut University, Faculty of Engineering, Vol. 41, No. 3, May, 2013, E-mail address: jes@aun.edu.eg 
L. K. Idriss et al., Static behaviour of different types of R.C beam-column connections as affected by both value acting lateral horizontal force and grade of used concrete (theoretical study) part two, pp.746 - 814

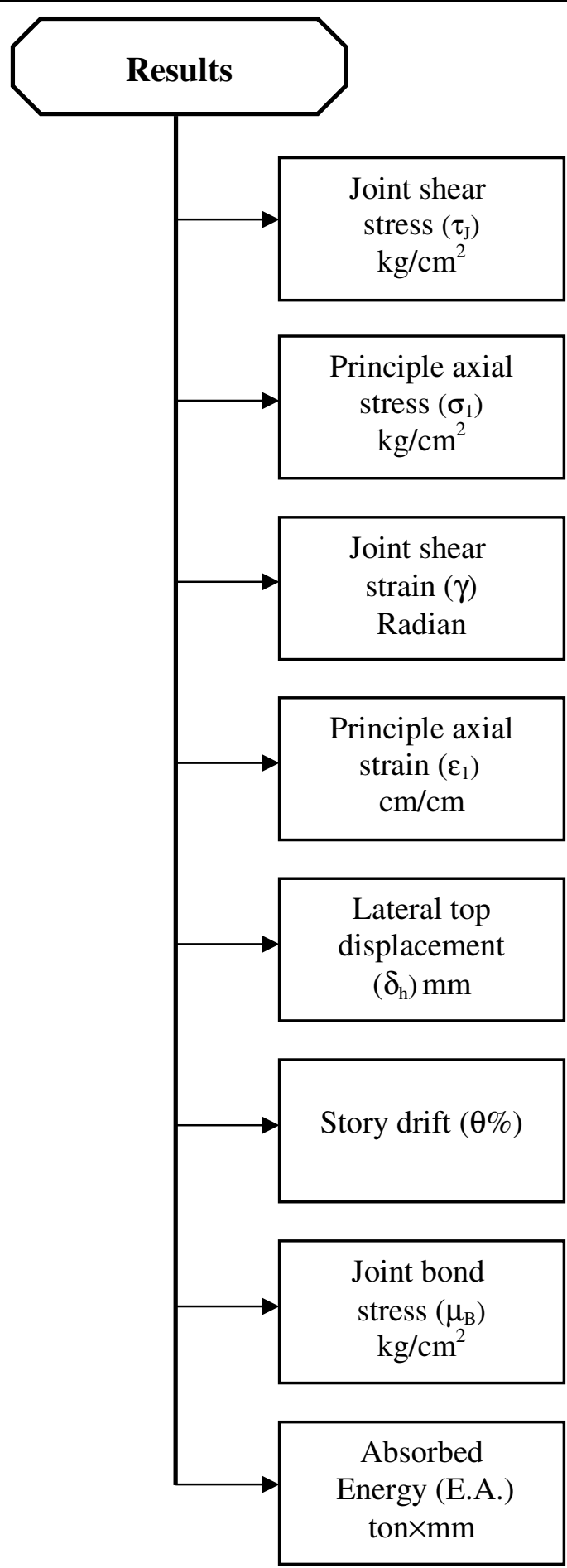

Journal of Engineering Sciences, Assiut University, Faculty of Engineering, Vol. 41, No. 3, May, 2013,E-mail address: jes@aun.edu.eg 
754

L. K. Idriss et al., Static behaviour of different types of R.C beam-column connections as affected by both value acting lateral horizontal force and grade of used concrete (theoretical study) part two, pp.746 - 814.

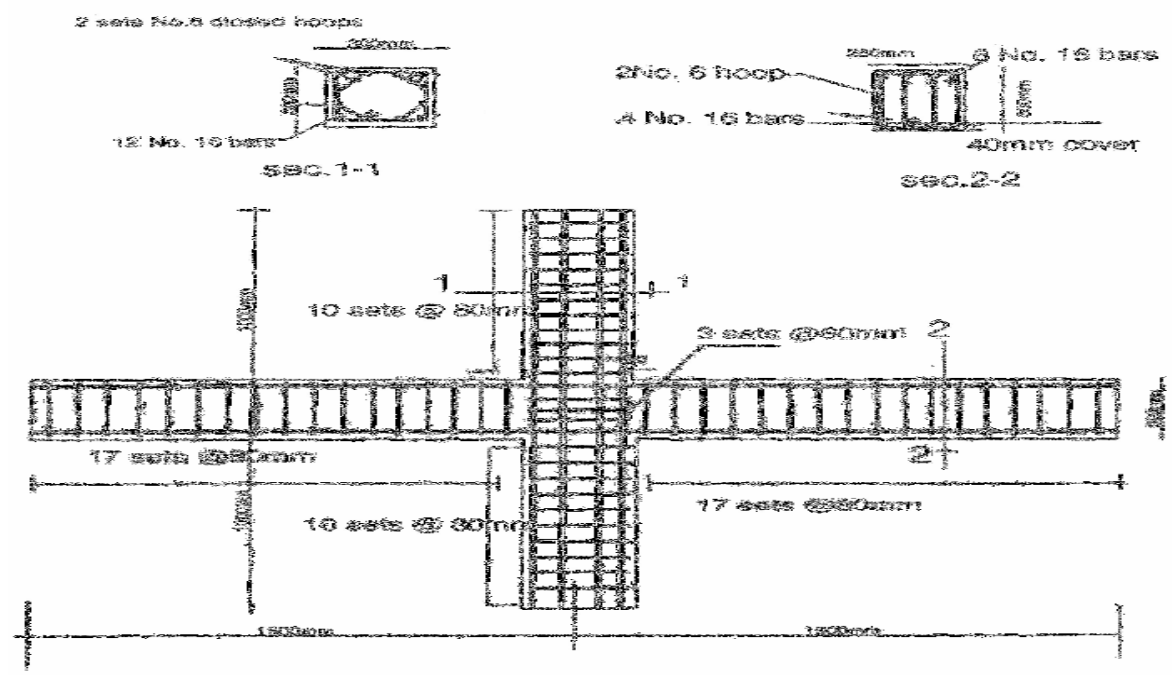

a) Reinforcement details of interior beam-column joint

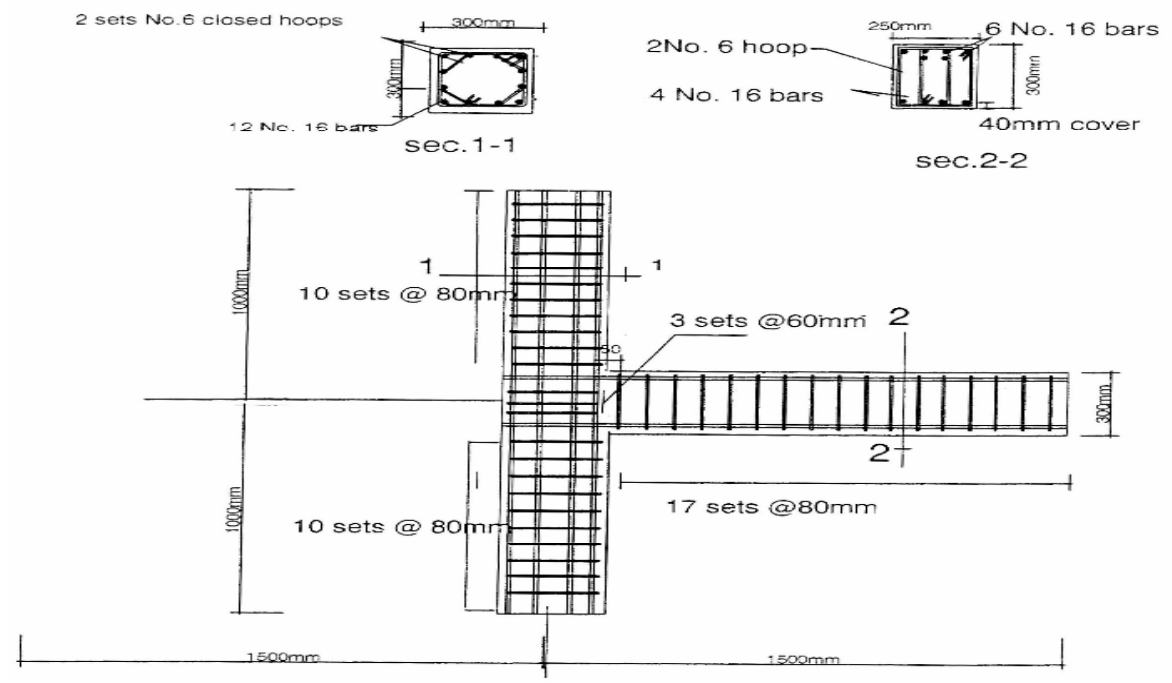

b) Reinforcement details of exterior beam-column joint

Journal of Engineering Sciences, Assiut University, Faculty of Engineering, Vol. 41, No. 3, May, 2013,E-mail address: jes@aun.edu.eg 
L. K. Idriss et al., Static behaviour of different types of R.C beam-column connections as affected by both value acting lateral horizontal force and grade of used concrete (theoretical study) part two, pp.746 - 814

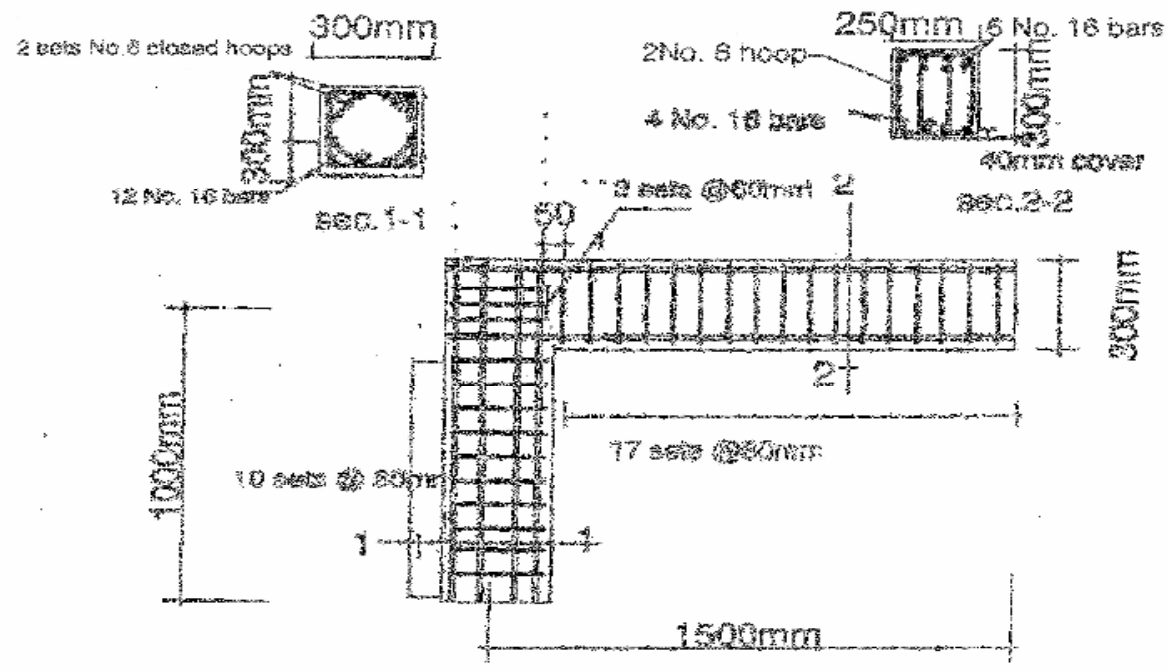

c) Reinforcement details of roof corner beam-column joint

Fig. 3. The loading set up and Boundary condition of joints

\section{Materials nonlinearity}

Steel and concrete are the basic materials used in the structural elements. To model the cyclic characteristics of the earthquake load, a nonlinear material model with specific cyclic features should be used for each.

Steel: In this article, an isotropic kinematics hardening model is used for steel material. As shown in Figure 4, the blushing effect has been taken into account, and there is no stiffness degradation during the cycling. It is acceptable for the skyscraper structure as the maximum steel strain should be less than $2.5 \%$.

Concrete: The plastic-damage model (J. Lee, 1998) is used to model the concrete material. The model is a continuum, plasticity-based, model for concrete. It assumes that the main two failure mechanisms are tensile cracking and compressive crushing of the concrete material. It captures the three major characteristics of the concrete in the buildings: (1) the strength of compression is larger than that of tension; (2) the stiffness degrades when it goes into plastic range; (3) the stiffness recovers when it reverses from tension to compression.

Journal of Engineering Sciences, Assiut University, Faculty of Engineering, Vol. 41, No. 3, May, 2013,E-mail address: jes@aun.edu.eg 
L. K. Idriss et al., Static behaviour of different types of R.C beam-column connections as affected by both value acting lateral horizontal force and grade of used concrete (theoretical study) part two, pp.746 - 814.

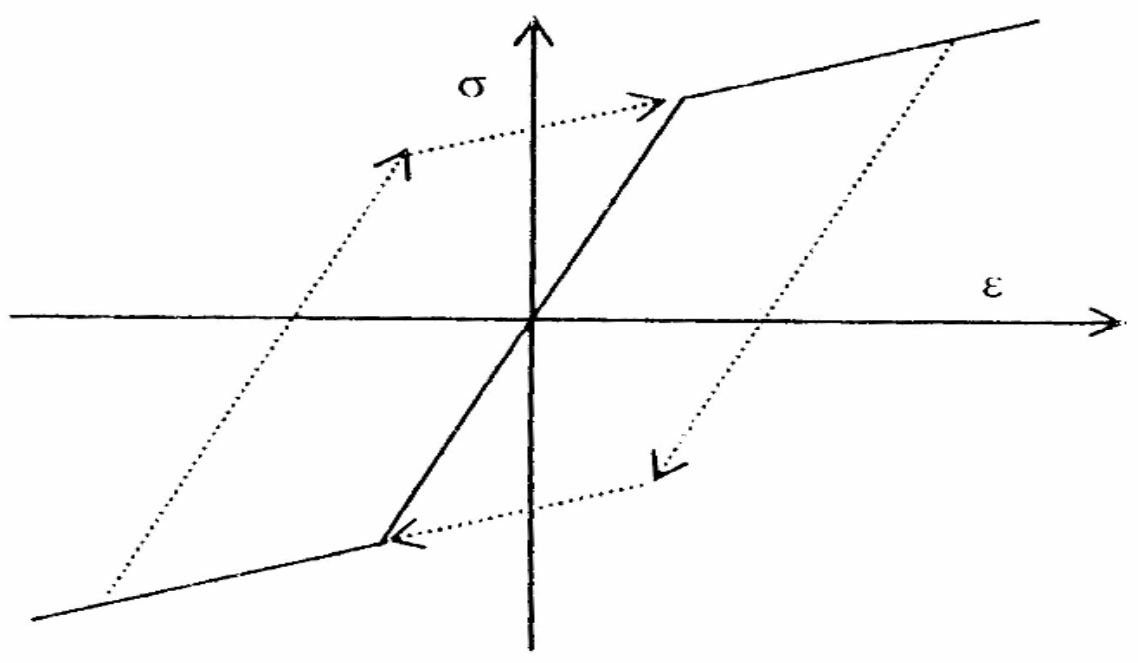

Fig.4. Steel constitute law (Sam Lee 2008)

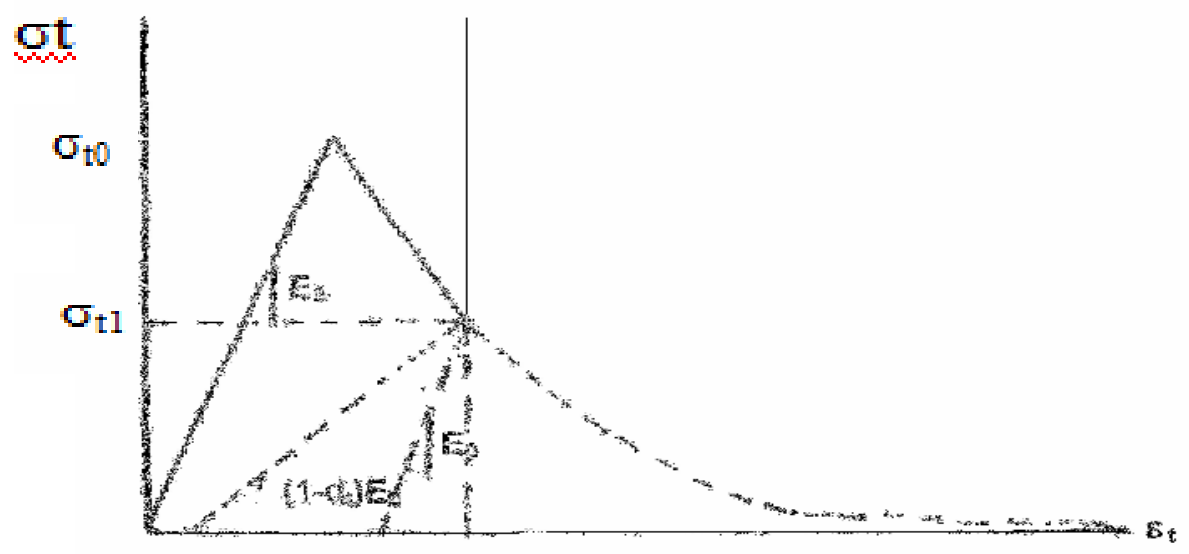

Fig. 5. Concrete in tension (Sam Lee 2008)

Journal of Engineering Sciences, Assiut University, Faculty of Engineering, Vol. 41, No. 3, May, 2013,E-mail address: jes@aun.edu.eg 
L. K. Idriss et al., Static behaviour of different types of R.C beam-column connections as affected by both value acting lateral horizontal force and grade of used concrete (theoretical study) part two, pp.746 - 814

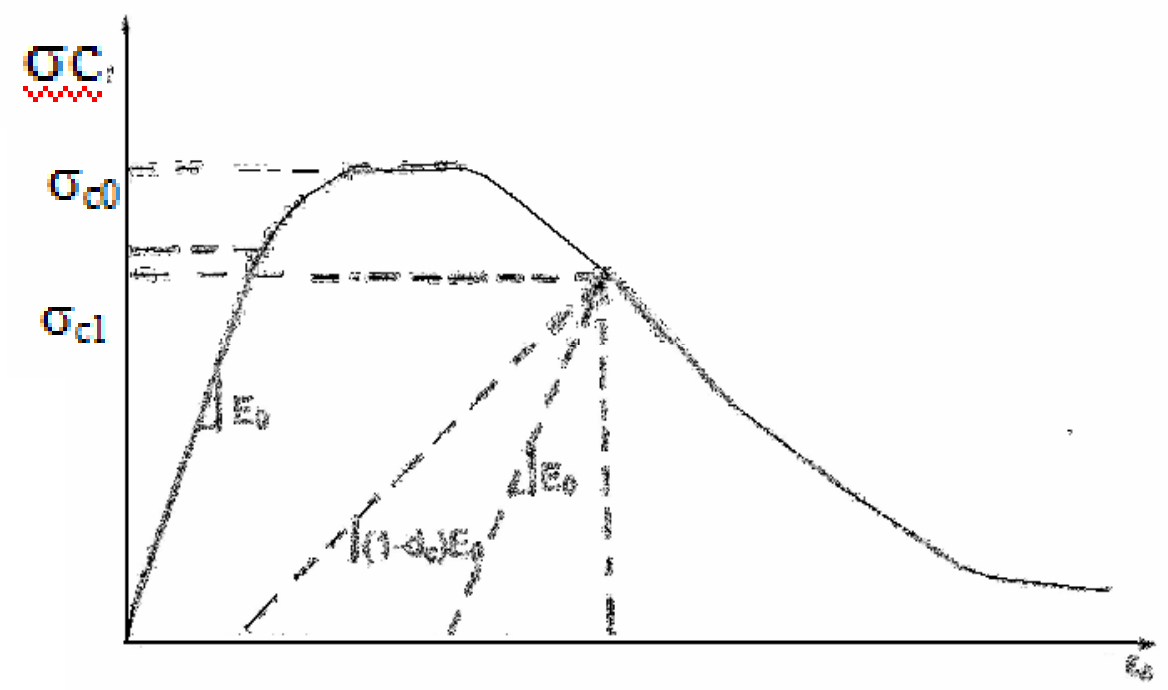

Fig. 6. Concrete in compression (Sam Lee 2008)

Figures 5 and 6 show the concrete material's stress-strain curves, the stiffness of the concrete degrades when it unloads from the plastic range. The degradation factors for compression (dc) and tension (dt) are dependent on the plastic strain (ABAQUS,V 6.5). Figure 7 shows the hysteric curve of the concrete, it can be seen that the stiffness recovers when the material stress status reverses from tension to compression.

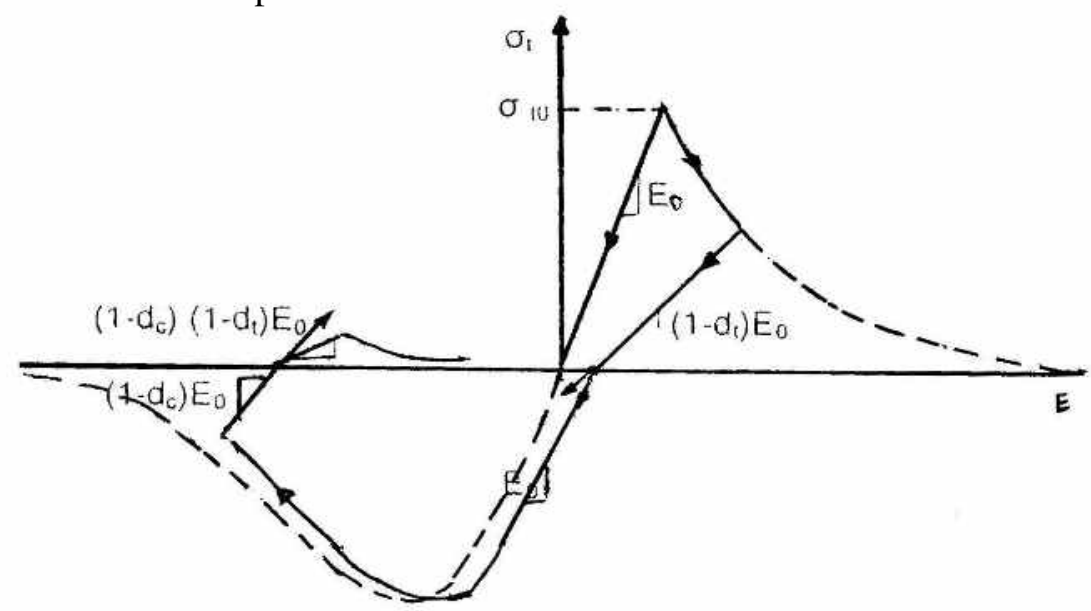

Fig.7. Concrete hysteric curve (Sam Lee 2008)

In the fixed crack model, the crack direction is determined and fixed at the time of crack initiation. In the rotating crack model, the crack direction is identical with a principal strain direction and rotates if the strain direction changes. The main difference in these crack models is Journal of Engineering Sciences, Assiut University, Faculty of Engineering, Vol. 41, No. 3, May, 2013,E-mail address: jes@aun.edu.eg 
L. K. Idriss et al., Static behaviour of different types of R.C beam-column connections as affected by both value acting lateral horizontal force and grade of used concrete (theoretical study) part two, pp.746 - 814 .

the absence of shear stresses on the crack plane in the rotating crack model due coincidence of principal strain directions with the crack orientation, which makes the rotating crack model more simple. In the fixed crack model the shear resistance of the cracks is modeled by means of the variable shear retention factor, which reflects the aggregate interlock effect of cracked concrete) Concrete in plane stress condition can be well described by a damage model such as the one used in the ABAQUS, see Figure 8. It is based on the "equivalent uniaxial law", which covers the complete range of the plane stress behaviour in tension and compression. The effect of biaxial stress state on the concrete strength is captured by the failure function due to (Kupfer et al., 2011). For the tensile response (cracking) the crack band method described above is applied. Similar method is applied for the compressive softening. Thus complete softening behaviour is based on an objective and mesh independe1nt approach.

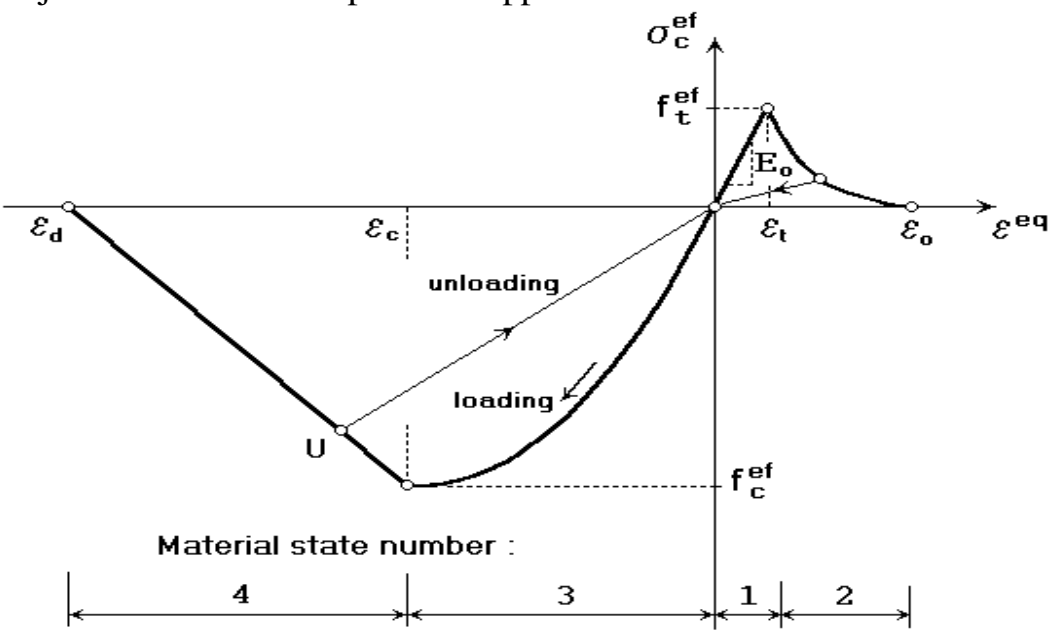

Fig. 8. a Equivalent uniaxial law (left)

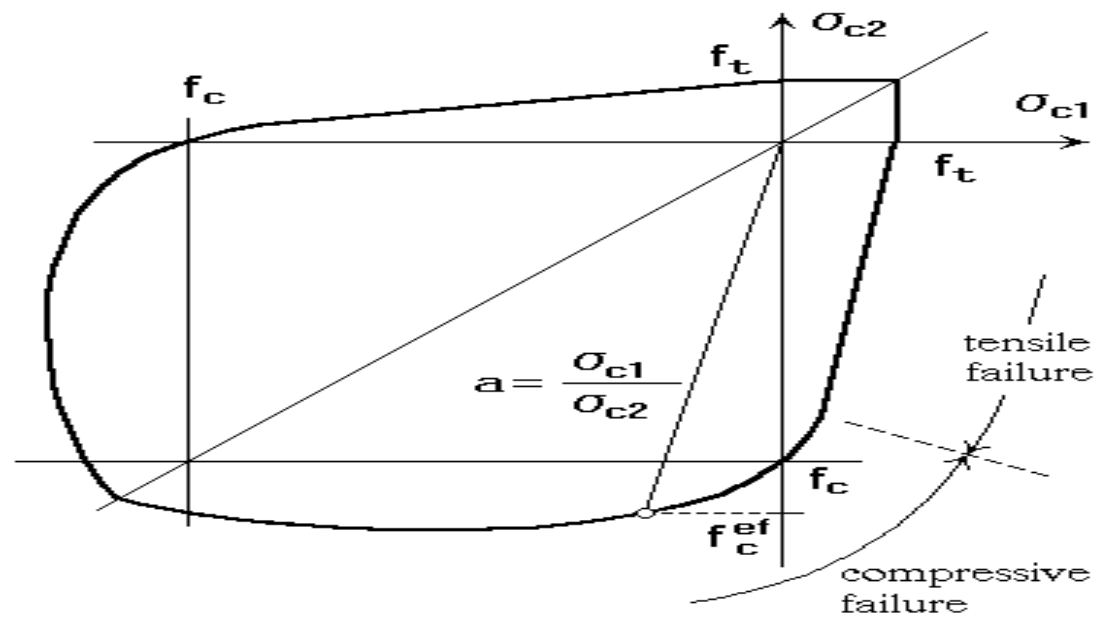

Fig. 8. b Bi-axial failure function by Kupfer2011

Journal of Engineering Sciences, Assiut University, Faculty of Engineering, Vol. 41, No. 3, May, 2013,E-mail address: jes@aun.edu.eg 
L. K. Idriss et al., Static behaviour of different types of R.C beam-column connections as affected by both value acting lateral horizontal force and grade of used concrete (theoretical study) part two, pp.746 - 814

\section{Data given and obtained results}

Figure 9 shows the 3-D meshed modeling connections with loading, boundary condition and deflection Shapes studying by ABAQUSICAE 6.7 software for interior, exterior and corner joints.

The obtained theoretically evaluated values of various stresses and displacements for both interior and exterior joints for the case study are tabulated and given in Tables (2),(3)and (4).
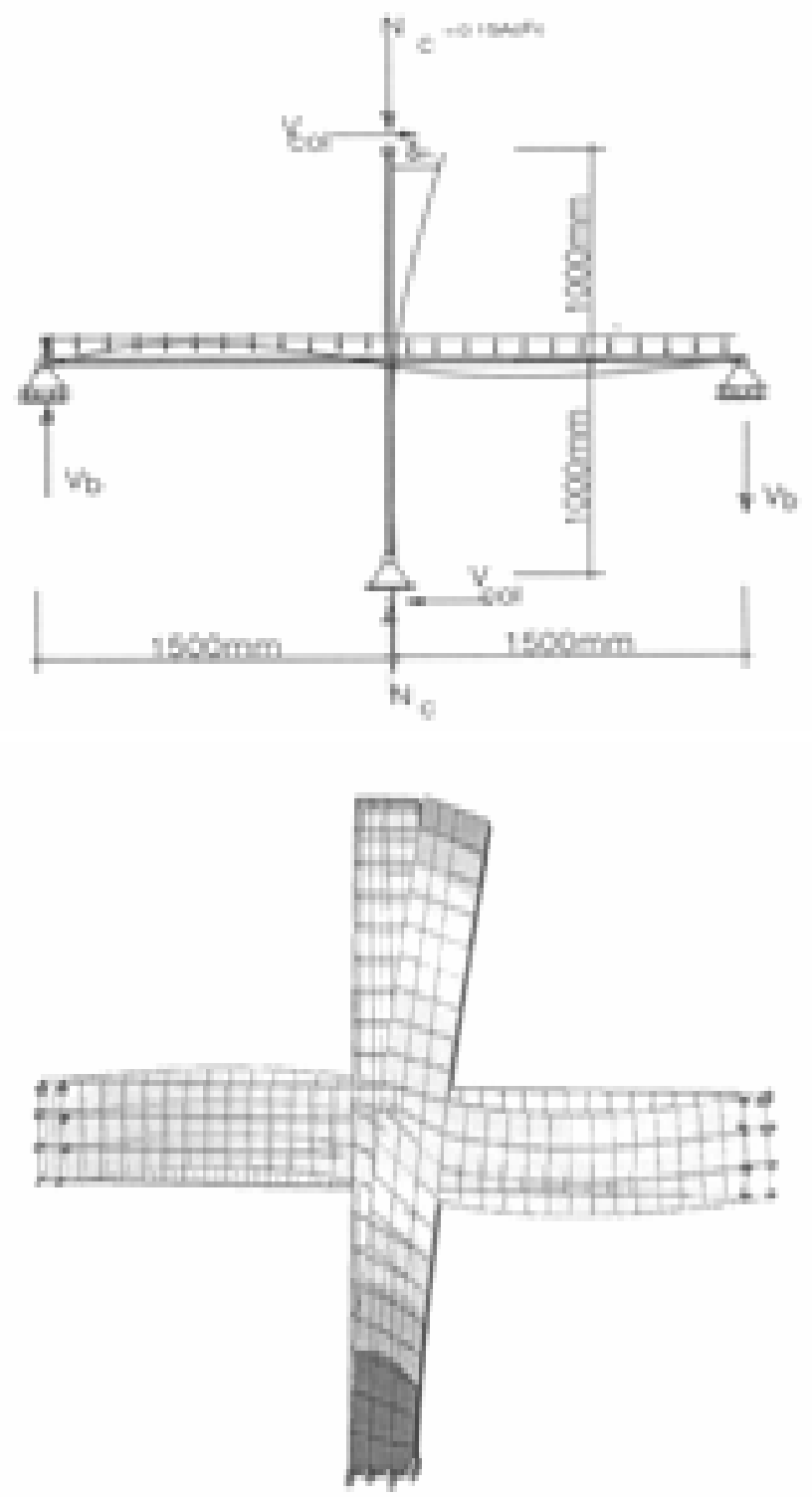

a) On interior joint

Journal of Engineering Sciences, Assiut University, Faculty of Engineering, Vol. 41, No. 3, May, 2013,E-mail address: jes@aun.edu.eg 
L. K. Idriss et al., Static behaviour of different types of R.C beam-column connections as affected by both value acting lateral horizontal force and grade of used concrete (theoretical study) part two, pp.746 - 814.
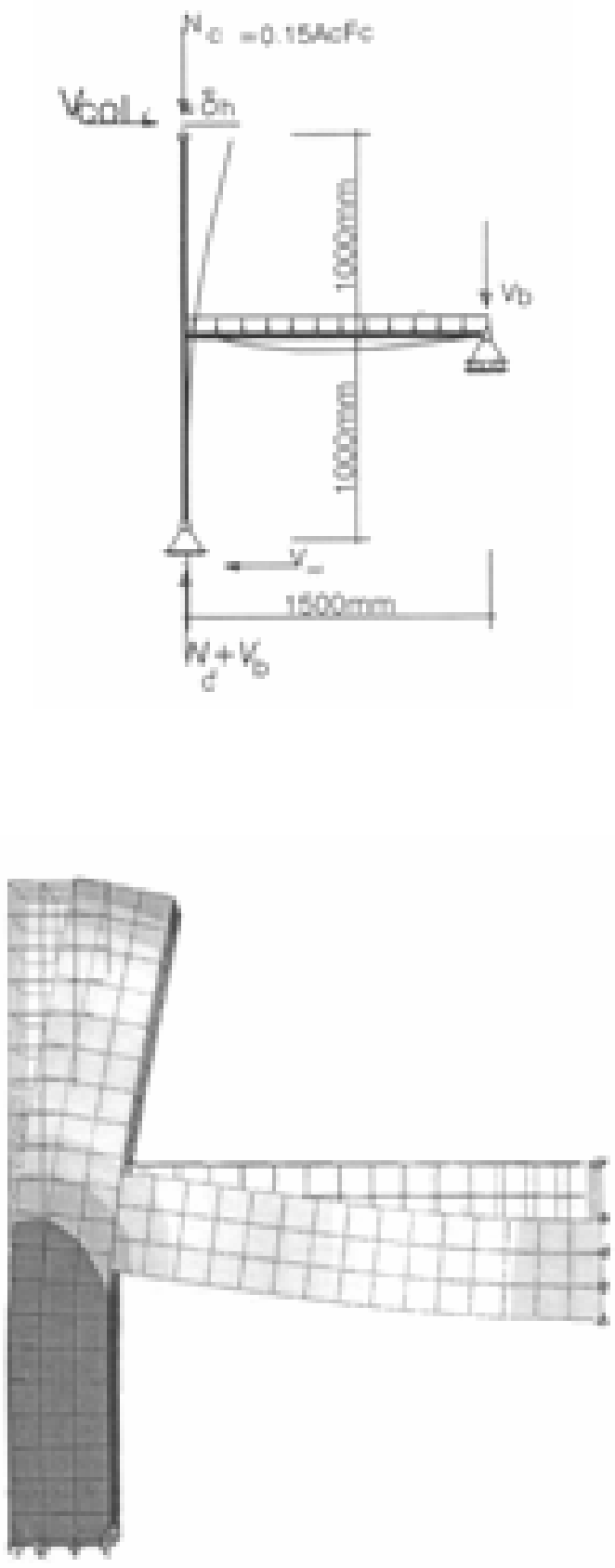

b) On exterior joint

Journal of Engineering Sciences, Assiut University, Faculty of Engineering, Vol. 41, No. 3, May, 2013,E-mail address: jes@aun.edu.eg 

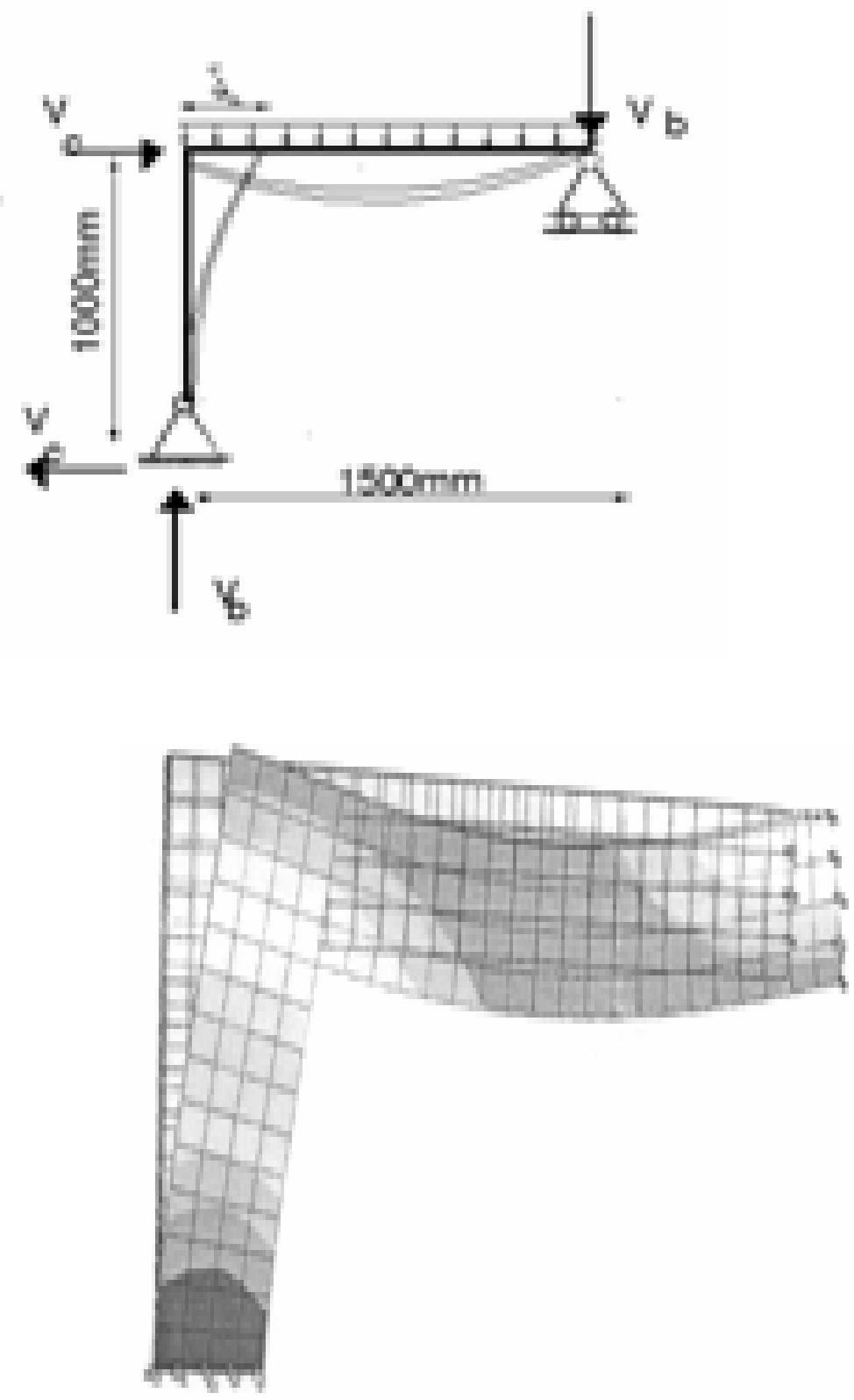

c) On knee joint

Fig.9. Finite element meshes and deflection shapes at definition boundary condition

Journal of Engineering Sciences, Assiut University, Faculty of Engineering, Vol. 41, No. 3, May, 2013,E-mail address: jes@aun.edu.eg 
762

L. K. Idriss et al., Static behaviour of different types of R.C beam-column connections as affected by both value acting lateral horizontal force and grade of used concrete (theoretical study) part two, pp.746 - 814.

The effect of the studied parameters on the behavior of beam-column joint are evaluated according to table 2,3and 4 with the following relationships:

\section{Relation of story shear force (Vc) ton - story drift $(\theta \%)$}

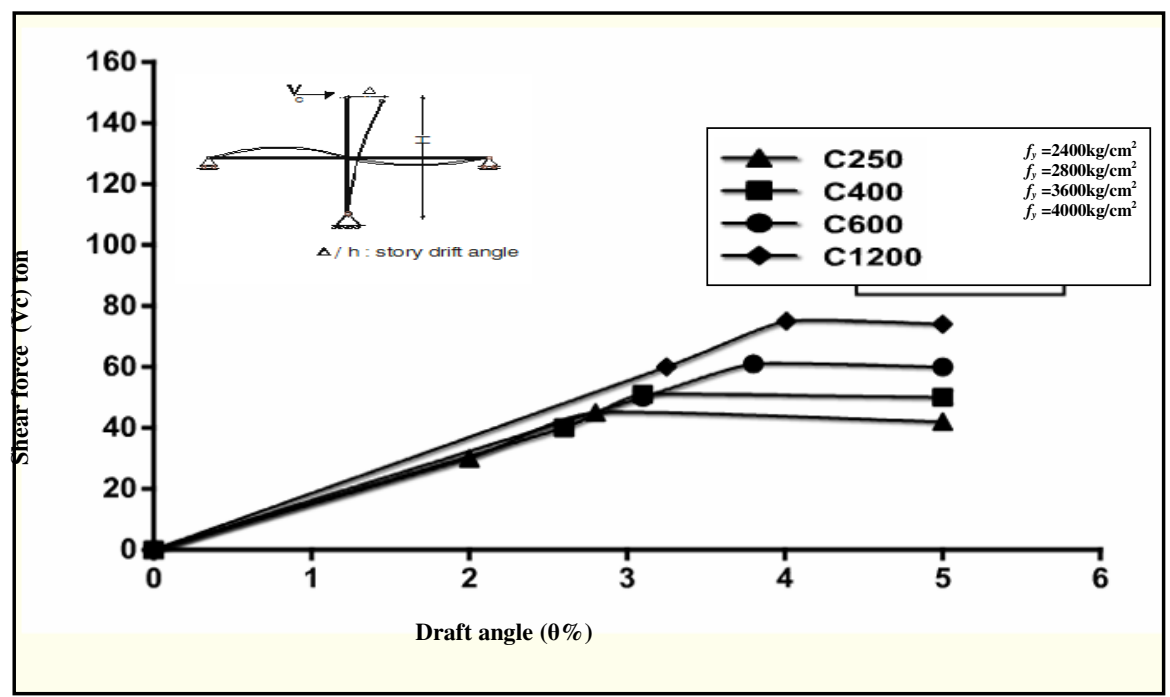

a) Relation of story shear force-story drift at interior joint

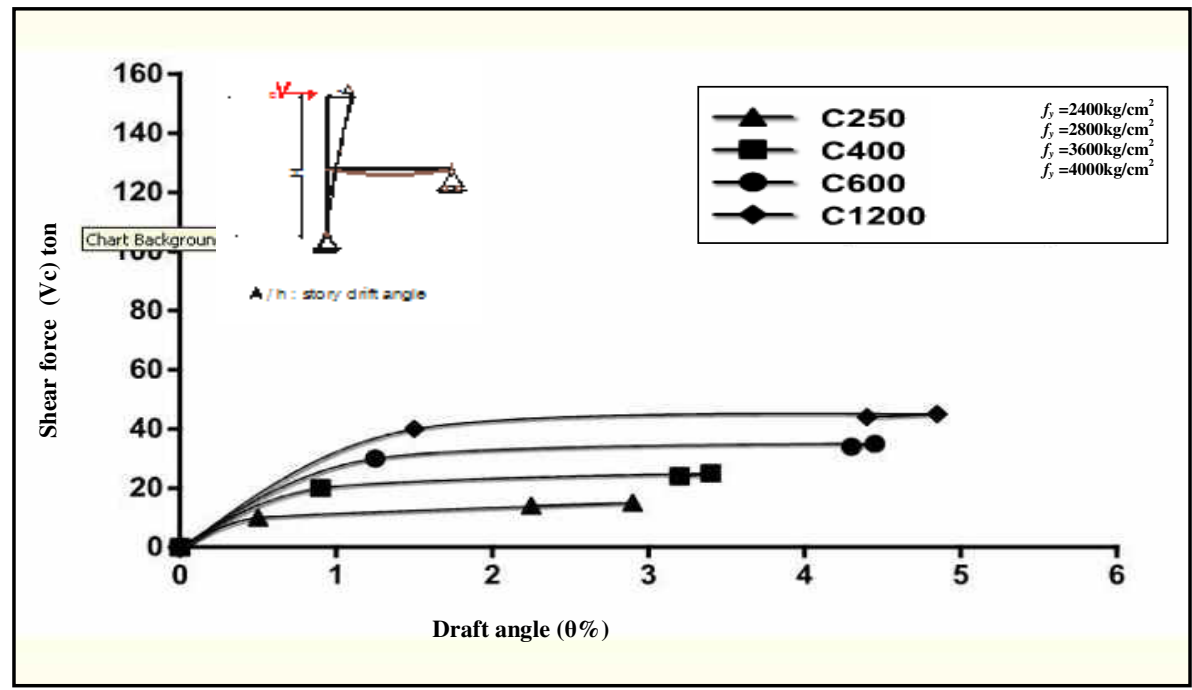

b) Relation of story shear force-story drift at exterior joint

Journal of Engineering Sciences, Assiut University, Faculty of Engineering, Vol. 41, No. 3, May, 2013, E-mail address: jes@aun.edu.eg 
L. K. Idriss et al., Static behaviour of different types of R.C beam-column connections as affected by both value acting lateral horizontal force and grade of used concrete (theoretical study) part two, pp.746 - 814

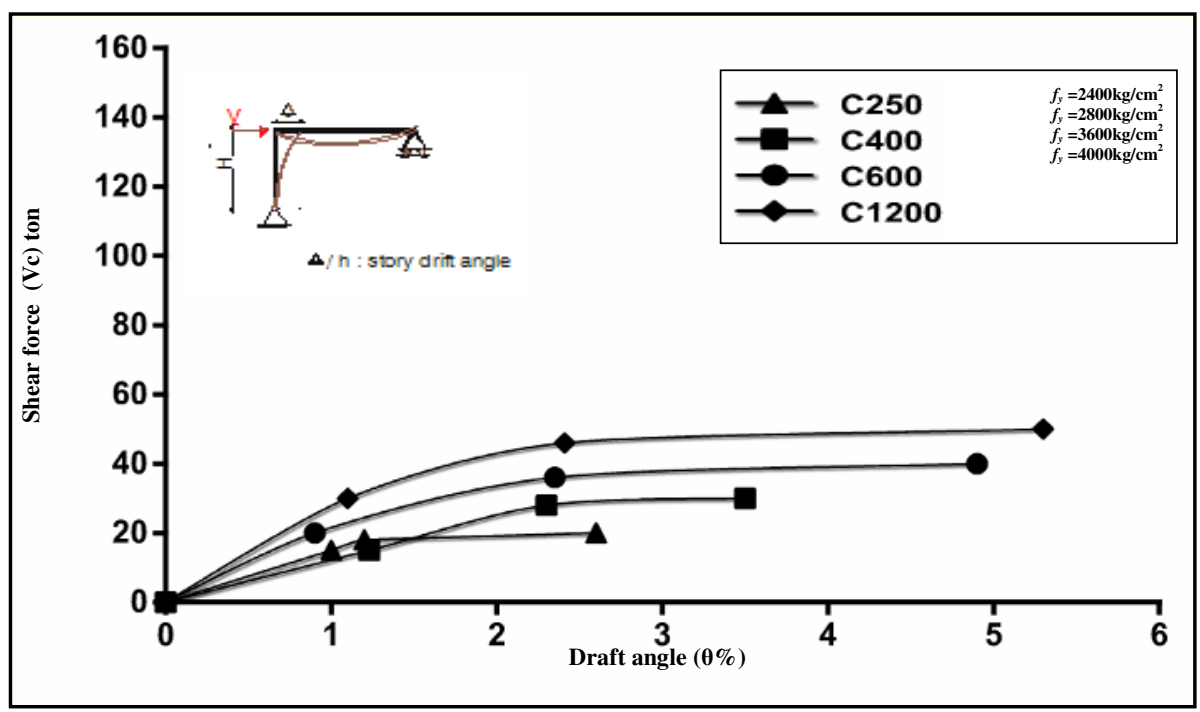

c) Relation of story shear force-story drift at corner joint

Fig. 10. Relation of story shear force $(\mathrm{Vc})$ - Story Drift $(\theta \%)$ for Beam-Column Joint

Fig.10 illustrate that in general as the story shear forces $\left(\mathrm{V}_{\mathrm{c}}\right)$ increase, the corresponding story drift angle $(\theta \%)$ increase.

2. Relation of joint shear stress $\left(\tau_{\mathrm{j}}\right) \mathrm{kg} / \mathrm{cm}^{2}$-story $\operatorname{drift}(\theta \%)$.

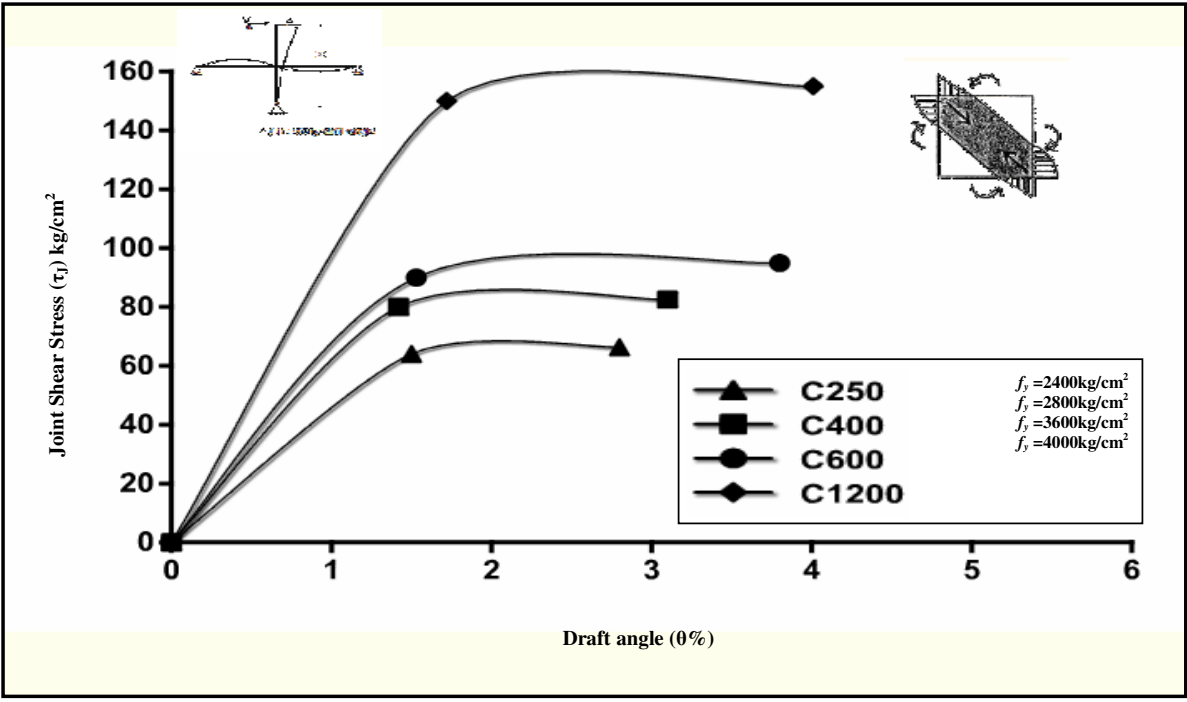

a) Relation of story shear stress - story drift at interior joint

Journal of Engineering Sciences, Assiut University, Faculty of Engineering, Vol. 41, No. 3, May, 2013,E-mail address: jes@aun.edu.eg 
L. K. Idriss et al., Static behaviour of different types of R.C beam-column connections as affected by both value acting lateral horizontal force and grade of used concrete (theoretical study) part two, pp.746 - 814.

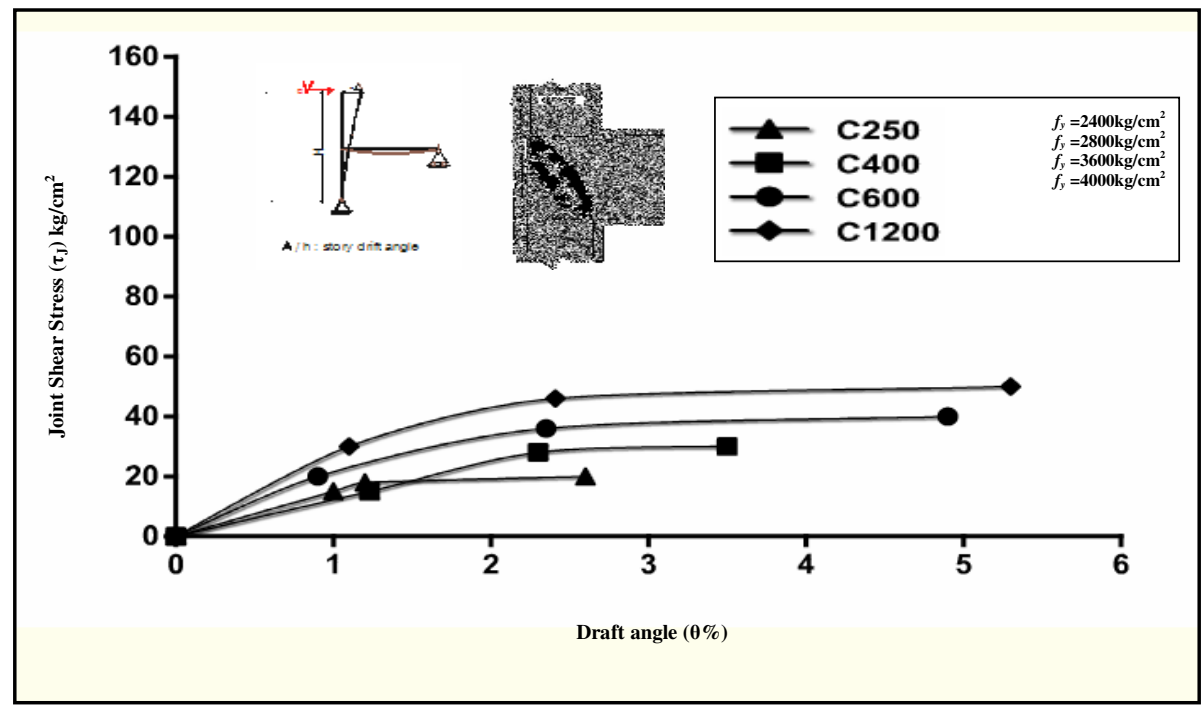

b) Relation of story shear stress - story drift at exterior joint

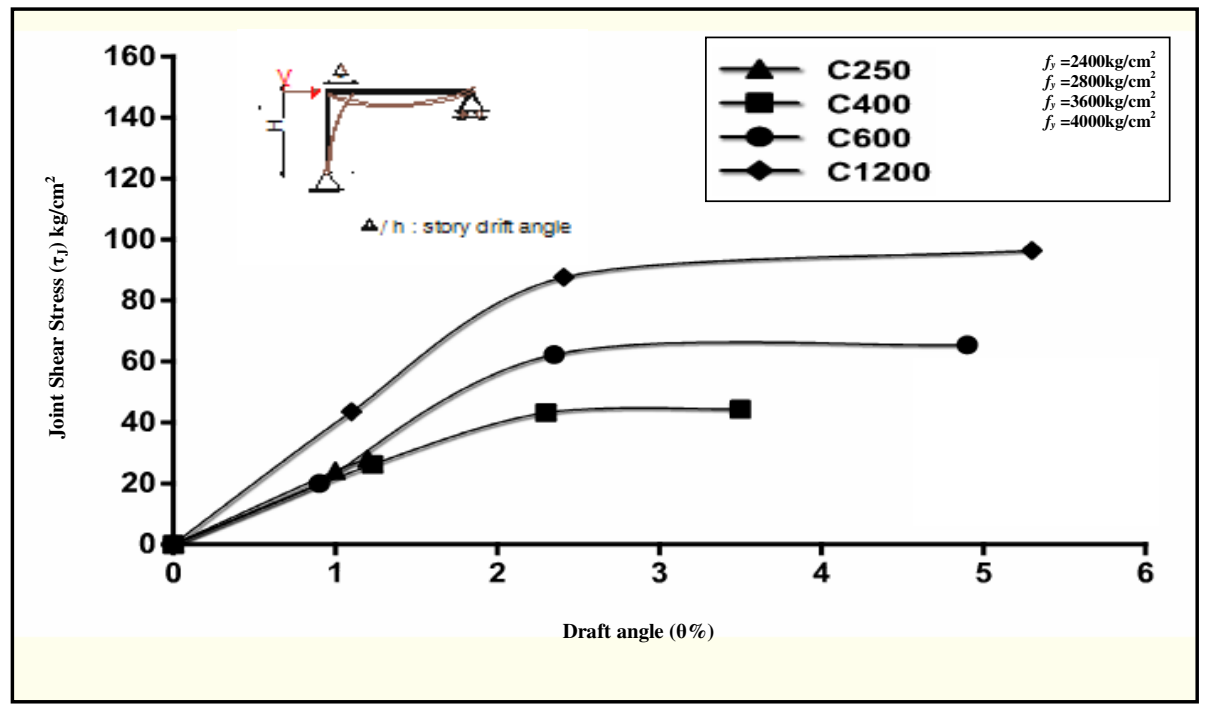

c) Relation of story shear stress-story drift at corner joint

Fig. 11. Relation of Story Shear $\left(\tau_{\mathrm{j}}\right)$ - Story Drift $(\theta \%)$ for Beam-Column Joint

It is observed at Fig.11 that the relation between joint shear stress $\left(\tau_{\mathrm{j}}\right)$ and story drift angle $(\theta \%)$ is leaner for story drift $(1-2) \%$.

Journal of Engineering Sciences, Assiut University, Faculty of Engineering, Vol. 41, No. 3, May, 2013,E-mail address: jes@aun.edu.eg 
L. K. Idriss et al., Static behaviour of different types of R.C beam-column connections as affected by both value acting lateral horizontal force and grade of used concrete (theoretical study) part two, pp.746 - 814

\section{Table 2.}

Obtained Theoretical Results for Studied Interior Beam-column Joints.

\begin{tabular}{|c|c|c|c|c|c|c|c|c|c|c|c|c|c|}
\hline $\begin{array}{c}\text { Joint } \\
\text { No. }\end{array}$ & $\begin{array}{c}\text { Compressive } \\
\text { concrete } \\
\left(f_{c}\right) \\
\mathbf{k g} / \mathrm{cm}^{2}\end{array}$ & $\begin{array}{c}\text { Yield } \\
\text { strength } \\
\left(f_{y}\right) \\
\mathbf{k g} / \mathrm{cm}^{2}\end{array}$ & $\begin{array}{c}\text { Axial } \\
\text { load } \\
\text { ton } \\
\left(\mathbf{N}_{\mathrm{c}}\right)\end{array}$ & $\begin{array}{l}\mathbf{N}_{\mathrm{c}} / \\
f_{\mathrm{c}^{\prime}} \\
\mathbf{A}_{\mathbf{c}}\end{array}$ & $\begin{array}{l}\text { Lateral } \\
\text { load } \\
\text { ton } \\
\left(V_{c}\right)\end{array}$ & $\begin{array}{l}\text { Draft } \\
\text { angle } \\
(\theta \%)\end{array}$ & $\begin{array}{c}\text { Top } \\
\text { lateral } \\
\text { Displace- } \\
\text { ment } \\
\text { mm }(\delta h) \\
\end{array}$ & $\begin{array}{c}\text { Max } \\
\text { principle } \\
\text { stress } \\
\mathrm{Kg} / \mathrm{cm}^{2} \\
\left(\sigma_{1}\right) \\
\end{array}$ & $\begin{array}{c}\text { Joint } \\
\text { shear } \\
\text { stress } \\
\text { kg/cm² } \\
\left(\tau_{\mathrm{J}}\right) \\
\end{array}$ & $\begin{array}{c}\text { Joint } \\
\text { bond } \\
\text { stress } \\
\mathrm{kg} / \mathrm{cm}^{2} \\
\left(\mu_{\mathrm{b}}\right) \\
\end{array}$ & $\begin{array}{c}\text { Max } \\
\text { principle } \\
\text { strain } \\
\mathrm{cm} / \mathrm{cm} \\
\left(\varepsilon_{1} \times \mathbf{1 0}^{-4}\right) \\
\end{array}$ & $\begin{array}{c}\text { Joint } \\
\text { shear } \\
\text { distortion } \\
\text { radian } \\
(\gamma \%) \\
\end{array}$ & $\begin{array}{c}\text { Energy } \\
\text { Absorption } \\
\text { (E.A) } \\
\text { ton } \times \mathbf{m m}\end{array}$ \\
\hline $\mathbf{J}(1)$ & 250 & 2400 & 0 & 0 & 0 & 0 & 0 & 0 & 0 & 0 & 0 & 0 & 0 \\
\hline $\mathbf{J}(2)$ & 250 & 2400 & 25 & 0.15 & 5 & 0.345 & 6 & 44.11 & 16.32 & 8.36 & 0.129 & 0.48 & 480 \\
\hline $\mathrm{J}(3)$ & 250 & 2400 & 40 & 0.15 & 11 & 0.8 & 12 & 102 & 37.75 & 16.60 & 0.256 & 1.11 & 1200 \\
\hline $\mathrm{J}(6)$ & 400 & 2800 & 25 & 0.15 & 7.5 & 0.45 & 6.40 & 70.32 & 35.32 & 14.60 & 0.23 & 0.6 & 440 \\
\hline $\mathbf{J}(7)$ & 400 & 2800 & 40 & 0.15 & 10 & 0.71 & 8.5 & 104.9 & 45.33 & 20.20 & 0.31 & 0.77 & 1580 \\
\hline $\mathbf{J}(8)$ & 400 & 2800 & 80 & 0.15 & 51 & 3.10 & 62 & 227.50 & 82.50 & 43.02 & 0.66 & 1.75 & 2959 \\
\hline $\mathbf{J}(9)$ & 600 & 3600 & 0 & 0 & 0 & 0 & 0 & 0 & 0 & 0 & 0 & 0 & 0 \\
\hline J (10) & 600 & 3600 & 40 & 0.15 & 13 & 0.84 & 5.2 & 135.8 & 53.31 & 35.39 & 0.34 & 0.88 & 450 \\
\hline $\mathrm{J}(15)$ & 1200 & 4000 & 120 & 0.15 & 22 & 1.12 & 14 & 317.9 & 106.8 & 82.64 & 0.65 & 1.50 & 2170 \\
\hline $\mathrm{J}(16)$ & 1200 & 4000 & 240 & 0.15 & 75 & 4.01 & 86 & 521.4 & 155.8 & 113.20 & 1.07 & 2.48 & 4083 \\
\hline
\end{tabular}

Journal of Engineering Sciences, Assiut University, Faculty of Engineering, Vol. 41, No. 3, May, 2013, E-mail address: jes@aun.edu.eg 
766

L. K. Idriss et al., Static behaviour of different types of R.C beam-column connections as affected by both value acting lateral horizontal force and grade of used concrete (theoretical study) part two, pp.746 - 814 .

\section{Table 3.}

Obtained Theoretical Results for Studied Exterior Beam-column Joints.

\begin{tabular}{|c|c|c|c|c|c|c|c|c|c|c|c|c|c|}
\hline $\begin{array}{c}\text { Joint } \\
\text { No. }\end{array}$ & $\begin{array}{c}\text { Compressive } \\
\text { concrete } \\
\left(f_{c}\right) \\
\mathbf{k g} / \mathrm{cm}^{2}\end{array}$ & $\begin{array}{c}\text { Yield } \\
\text { strength } \\
\left(f_{y}\right) \\
\mathrm{kg} / \mathrm{cm}^{2}\end{array}$ & $\begin{array}{c}\text { Axial } \\
\text { load } \\
\text { ton } \\
\mathrm{N}_{\mathrm{c}}\end{array}$ & $\begin{array}{l}\mathbf{N}_{\mathrm{c}} / \\
f_{\mathrm{c}} \\
\mathbf{A}_{\mathrm{c}}\end{array}$ & $\begin{array}{c}\text { Lateral } \\
\text { load } \\
\text { ton } \\
\left(V_{c}\right)\end{array}$ & $\begin{array}{c}\text { Draft } \\
\text { angle } \\
(\theta \\
\%)\end{array}$ & $\begin{array}{c}\text { Top } \\
\text { lateral } \\
\text { Displace- } \\
\text { ment } \\
\text { mm }(\delta \mathrm{h}) \\
\end{array}$ & $\begin{array}{c}\text { Max. } \\
\text { principle } \\
\text { stress } \\
\text { kg/cm² } \\
\left(\sigma_{1}\right) \\
\end{array}$ & $\begin{array}{c}\text { Joint } \\
\text { shear } \\
\text { stress } \\
\text { kg/cm } \\
\left(\tau_{\mathrm{J}}\right) \\
\end{array}$ & $\begin{array}{c}\text { Joint } \\
\text { bond } \\
\text { stress } \\
\text { Kg/cm² } \\
\left(\mu_{\mathrm{b}}\right) \\
\end{array}$ & $\begin{array}{c}\text { Max. } \\
\text { principle } \\
\text { strain } \\
\text { cm/cm } \\
\left(\varepsilon_{1} \times 10^{-4}\right)\end{array}$ & $\begin{array}{c}\text { Joint } \\
\text { shear } \\
\text { distortion } \\
\text { radian } \\
(\gamma \%) \\
\end{array}$ & $\begin{array}{c}\text { Energy } \\
\text { Absorption } \\
\text { (E.A) } \\
\text { ton } \times \mathbf{m m}\end{array}$ \\
\hline $\mathrm{J}(17)$ & 250 & 2400 & 0 & 0 & 0 & 0 & 0 & 0 & 0 & 0 & 0 & 0 & 0 \\
\hline $\mathrm{J}(18)$ & 250 & 2400 & 15 & 0.15 & 10 & 0.5 & 8.16 & 40 & 20 & 10.71 & 0.15 & 0.12 & 190 \\
\hline J (19) & 250 & 2400 & 30 & 0.15 & 13 & 0.79 & 12.72 & 57.5 & 23.70 & 24.09 & 0.603 & 0.48 & 390 \\
\hline $\mathbf{J}(20)$ & 250 & 2400 & 50 & 0.15 & 15 & 2.913 & 18.26 & 65 & 32.50 & 25.20 & 1.08 & 1.70 & 690 \\
\hline $\mathbf{J}(21)$ & 400 & 2800 & 0 & 0 & 0 & 0 & 0 & 0 & 0 & 0 & 0 & 0 & 0 \\
\hline $\mathrm{J}(22)$ & 400 & 2800 & 25 & 0.15 & 15 & 0.66 & 9.79 & 48.75 & 26.40 & 15.72 & 0.22 & 0.17 & 305 \\
\hline $\mathbf{J}(23)$ & 400 & 2800 & 40 & 0.15 & 23 & 1.01 & 18.58 & 75 & 40 & 35.32 & 1.03 & 0.80 & 620 \\
\hline $\mathrm{J}(24)$ & 400 & 2800 & 80 & 0.15 & 25 & 3.40 & 21.58 & 80.62 & 46.63 & 35.66 & 1.19 & 2.20 & 1098 \\
\hline $\mathbf{J}(25)$ & 600 & 3600 & 0 & 0. & 0 & 0 & 0 & 0 & 0 & 0 & 0 & 0 & 0 \\
\hline $\mathrm{J}(26)$ & 600 & 3600 & 40 & 0.15 & 20 & 0.81 & 10.68 & 64.50 & 49.45 & 39.85 & 0.93 & 0.67 & 430 \\
\hline J (27) & 600 & 3600 & 60 & 0.15 & 32 & 1.29 & 18.92 & 81.57 & 62.65 & 43.72 & 3.75 & 2.70 & 860 \\
\hline $\mathrm{J}(28)$ & 600 & 3600 & 120 & 0.15 & 35 & 4.45 & 28.67 & 214.6 & 64 & 44.25 & 3.89 & 2.80 & 1520 \\
\hline J (29) & 1200 & 4000 & 0 & 0 & 0 & 0 & 0 & 0 & 0 & 0 & 0 & 0 & 0 \\
\hline $\mathbf{J}(30)$ & 1200 & 4000 & 80 & 0.15 & 25 & 0.89 & 13.75 & 97.62 & 82.36 & 53.87 & 1.05 & 0.93 & 850 \\
\hline $\mathbf{J}(31)$ & 1200 & 4000 & 120 & 0.15 & 40 & 1.43 & 22.44 & 122.38 & 107.2 & 54.62 & 1.25 & 1.10 & 1750 \\
\hline J (32) & 1200 & 4000 & 240 & 0.15 & 45 & 4.85 & 32.62 & 318 & 116 & 55.54 & 4.20 & 3.70 & 2082 \\
\hline
\end{tabular}

Journal of Engineering Sciences, Assiut University, Faculty of Engineering, Vol. 41, No. 3, May, 2013, E-mail address: jes@aun.edu.eg 
L. K. Idriss et al., Static behaviour of different types of R.C beam-column connections as affected by both value acting lateral horizontal force and grade of used concrete (theoretical study) part two, pp.746 - 814

\section{Table 4.}

Obtained Theoretical Results for Studied Corner (Knee) Beam-column Joints.

\begin{tabular}{|c|c|c|c|c|c|c|c|c|c|c|c|}
\hline $\begin{array}{c}\text { Joint } \\
\text { No. }\end{array}$ & $\begin{array}{c}\text { Compressive } \\
\text { concrete } \\
\left(f_{c}\right) \mathrm{kg} / \mathrm{cm}^{2}\end{array}$ & $\begin{array}{c}\text { Yield } \\
\text { strength } \\
\left(f_{y}\right) \\
\mathbf{k g} / \mathrm{cm}^{2}\end{array}$ & $\begin{array}{c}\text { Lateral } \\
\text { load } \\
\left(V_{c}\right) \text { ton }\end{array}$ & $\begin{array}{l}\text { Draft } \\
\text { angle } \\
(\theta \%)\end{array}$ & $\begin{array}{l}\text { Top lateral } \\
\text { Displacement } \\
(\delta \mathrm{h}) \mathrm{mm}\end{array}$ & $\begin{array}{c}\text { Max } \\
\text { principle } \\
\text { stress } \\
\left(\sigma_{1}\right) \\
\text { Kg/cm } \\
\end{array}$ & $\begin{array}{c}\text { Joint } \\
\text { shear } \\
\text { stress } \\
\left(\tau_{\mathrm{J}}\right) \\
\mathrm{Kg} / \mathrm{cm}^{2} \\
\end{array}$ & $\begin{array}{c}\text { Joint } \\
\text { bond } \\
\text { stress } \\
\left(\mu_{\mathrm{b}}\right) \\
\mathbf{k g l c m}^{2} \\
\end{array}$ & $\begin{array}{c}\text { Max } \\
\text { principle } \\
\text { strain } \\
\varepsilon_{1} \times 10^{-4} \\
\mathrm{~cm} / \mathrm{cm} \\
\end{array}$ & $\begin{array}{c}\text { Joint } \\
\text { shear } \\
\text { distortion } \\
(\gamma \%) \\
\text { radian } \\
\end{array}$ & $\begin{array}{c}\text { Energy } \\
\text { Absorption } \\
\text { (E.A) } \\
\text { Ton } \times \mathbf{m m}\end{array}$ \\
\hline $\mathbf{J}(33)$ & 250 & 2400 & 0 & 0 & 0 & 5 & 0 & 0 & 0 & 0 & 0 \\
\hline J (34) & 250 & 2400 & 15 & 1.0 & 10 & 87.70 & 24.20 & 16.54 & 0.62 & 0.73 & 105 \\
\hline $\mathrm{J}(35)$ & 250 & 2400 & 18 & 1.2 & 12.7 & 102.3 & 28.10 & 18.32 & 0.74 & 0.86 & 223 \\
\hline $\mathrm{J}(36)$ & 250 & 2400 & 20 & 2.6 & 22.29 & 122.95 & 29.77 & 19.69 & 1.56 & 1.82 & 395 \\
\hline J (37) & 400 & 2800 & 0 & 0 & 0 & 10 & 0 & 0 & 0 & 0 & 0 \\
\hline J (38) & 400 & 2800 & 15 & 1.23 & 12.3 & 75.53 & 26.20 & 25.30 & 0.71 & 0.67 & 150 \\
\hline J (39) & 400 & 2800 & 28 & 2.3 & 23.0 & 125.1 & 43.20 & 28.67 & 1.15 & 1.10 & 300 \\
\hline $\mathrm{J}(40)$ & 400 & 2800 & 30 & 3.5 & 35.67 & 145.50 & 44.30 & 30.59 & 2.73 & 2.60 & 540 \\
\hline $\mathrm{J}(41)$ & 600 & 3600 & 0 & 0 & 0 & 0 & 0 & 0 & 0 & .0 & 0 \\
\hline $\mathrm{J}(42)$ & 600 & 3600 & 20 & 0.8 & 8.0 & 82.92 & 22.48 & 35.20 & 0.63 & 0.562 & 200 \\
\hline $\mathrm{J}(43)$ & 600 & 3600 & 36 & 2.35 & 22.5 & 233.67 & 62.26 & 46.76 & 2.03 & 1.67 & 400 \\
\hline J (44) & 600 & 3600 & 40 & 4.9 & 49.05 & 245.9 & 65.47 & 48.33 & 4.06 & 3.42 & 715 \\
\hline $\mathrm{J}(45)$ & 1200 & 4000 & 0 & 0 & 0 & 0 & 0 & 0 & 0 & 0 & 0 \\
\hline $\mathrm{J}(46)$ & 1200 & 4000 & 30 & 1.10 & 11.0 & 146.20 & 43.50 & 52.63 & 1.05 & 0.934 & 420 \\
\hline J (47) & 1200 & 4000 & 46 & 2.41 & 22.0 & 296.12 & 87.65 & 53.42 & 2.15 & 1.89 & 850 \\
\hline J (48) & 1200 & 4000 & 50 & 5.30 & 53.51 & 323.9 & 96.40 & 65.90 & 5.35 & 4.67 & 1552 \\
\hline
\end{tabular}

Journal of Engineering Sciences, Assiut University, Faculty of Engineering, Vol. 41, No. 3, May, 2013, E-mail address: jes@aun.edu.eg 
L. K. Idriss et al., Static behaviour of different types of R.C beam-column connections as affected by both value acting lateral horizontal force and grade of used concrete (theoretical study) part two, pp.746 - 814.

\section{Relation of Lateral Load $\left(V_{c}\right)$-Lateral Top displacement $\left(\delta_{h}\right)$.}

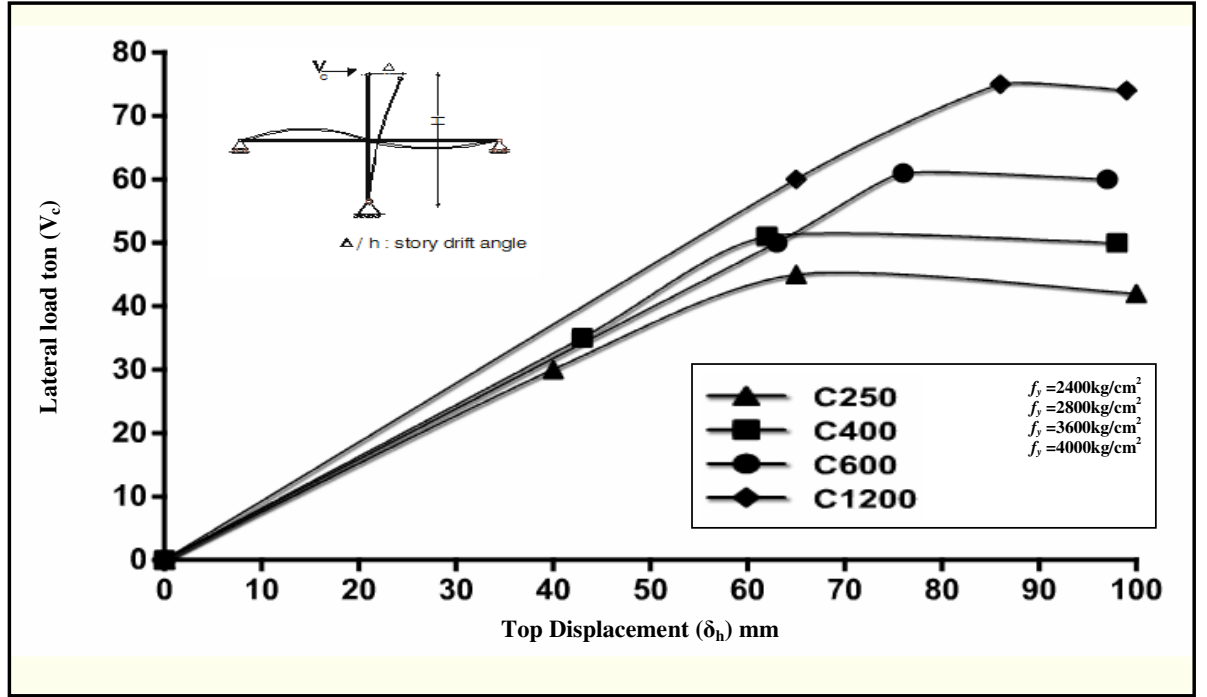

a) Relation of story shear force - lateral top displacement at interior joint

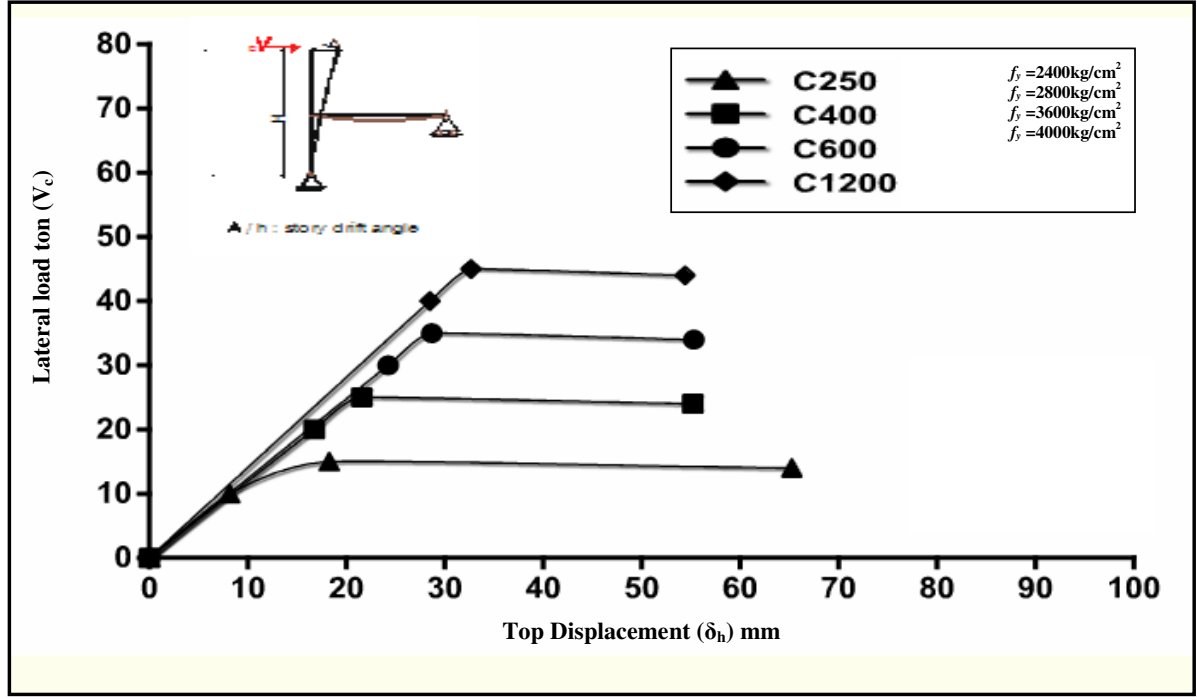

b) Relation of story shear force - lateral top displacement at exterior joint

Journal of Engineering Sciences, Assiut University, Faculty of Engineering, Vol. 41, No. 3, May, 2013,E-mail address: jes@aun.edu.eg 
L. K. Idriss et al., Static behaviour of different types of R.C beam-column connections as affected by both value acting lateral horizontal force and grade of used concrete (theoretical study) part two, pp.746 - 814

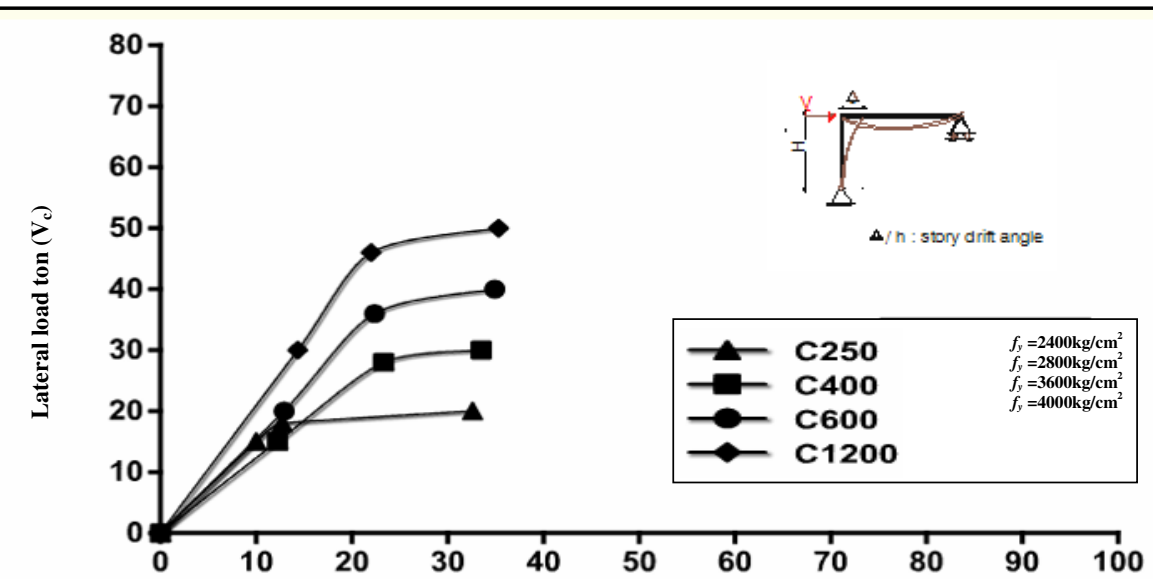

c) Relation of stor yisheemestrossmmlateral top displacement at corner joints

Fig. 12. Relation of Story Shear Force $\left(\mathrm{V}_{\mathrm{c}}\right)$ - Lateral Top Displacement $\left(\delta_{\mathrm{h}}\right)$ for Beam-Column Joints

Fig.12 illustrates that in general as the story shear forces $\left(\mathrm{V}_{\mathrm{c}}\right)$ increase, the corresponding lateral top displacement $\left(\delta_{\mathrm{h}}\right)$ increase.

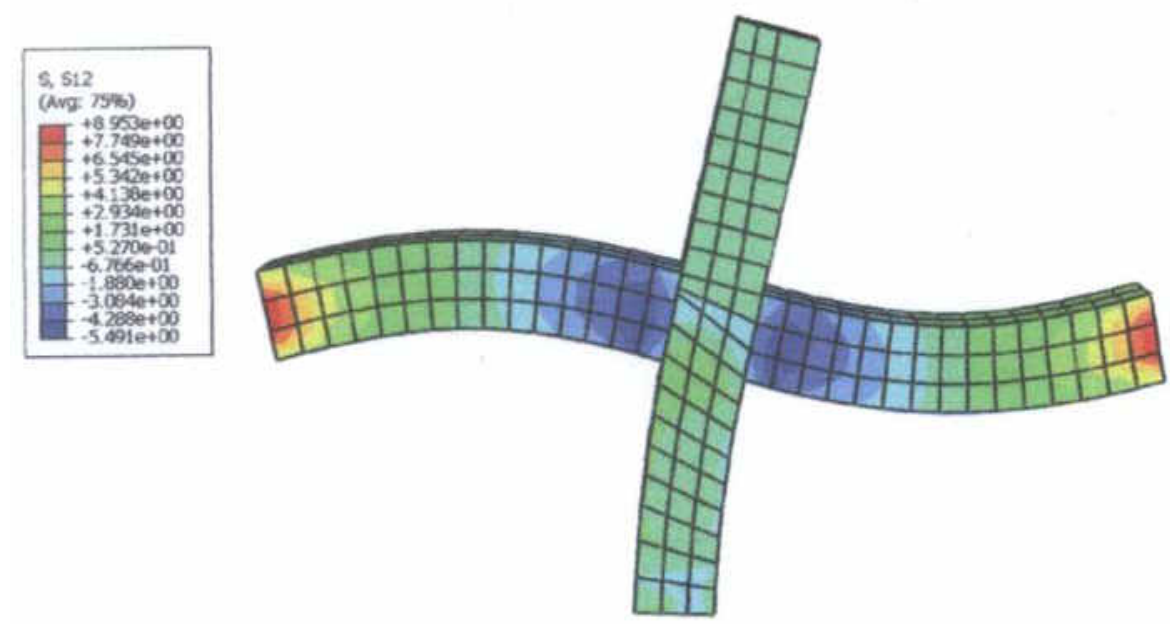

a) Shear stress at C250 Joint No. (2) for interior joint

Journal of Engineering Sciences, Assiut University, Faculty of Engineering, Vol. 41, No. 3, May, 2013,E-mail address: jes@aun.edu.eg 
L. K. Idriss et al., Static behaviour of different types of R.C beam-column connections as affected by both value acting lateral horizontal force and grade of used concrete (theoretical study) part two, pp.746 - 814.
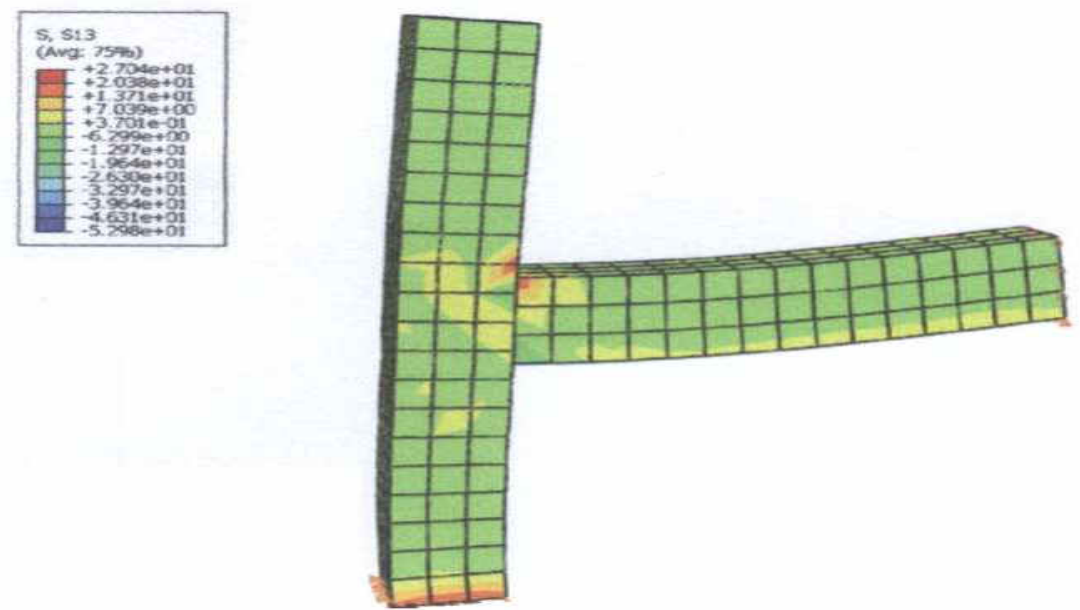

b) Shear stress at C600 Joint No. (28) for exterior joint
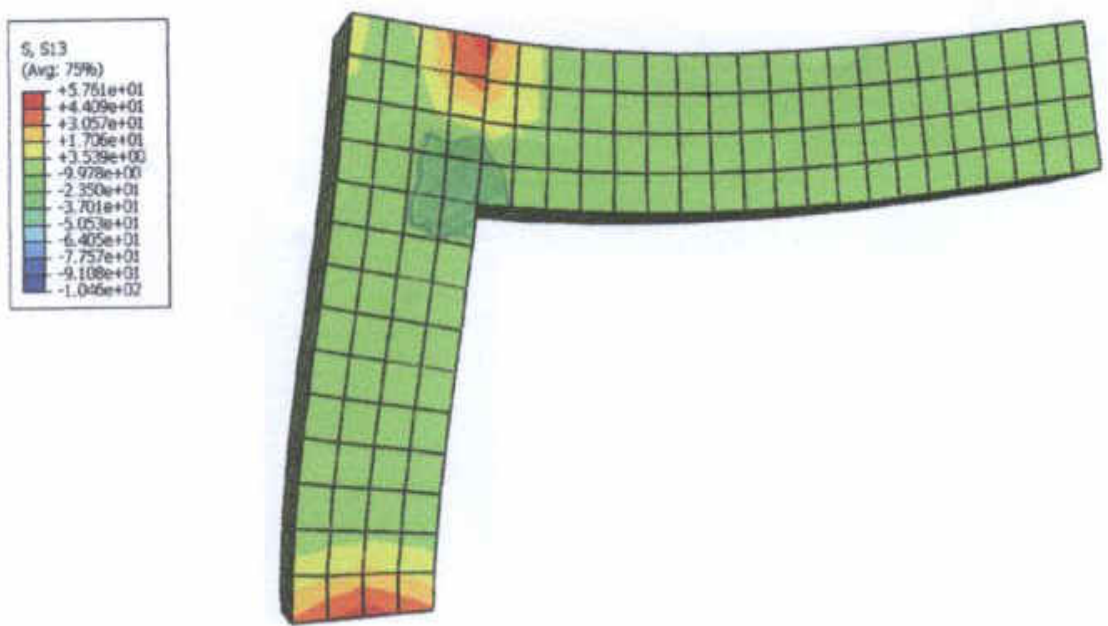

c) Shear stress at C1200 Joint No. (48) for knee joint

Fig. 13. Different Joint Shear Stresses at Different Grade of Concrete for joints

Journal of Engineering Sciences, Assiut University, Faculty of Engineering, Vol. 41, No. 3, May, 2013,E-mail address: jes@aun.edu.eg 
L. K. Idriss et al., Static behaviour of different types of R.C beam-column connections as affected by both value acting lateral horizontal force and grade of used concrete (theoretical study) part two, pp.746 - 814

\section{Relation of Joint Shear Stress $\left(\tau_{\mathrm{j}}\right) \mathrm{kg} / \mathrm{cm}^{2}$-Joint Shear $\operatorname{strain}(\gamma \%)$.}

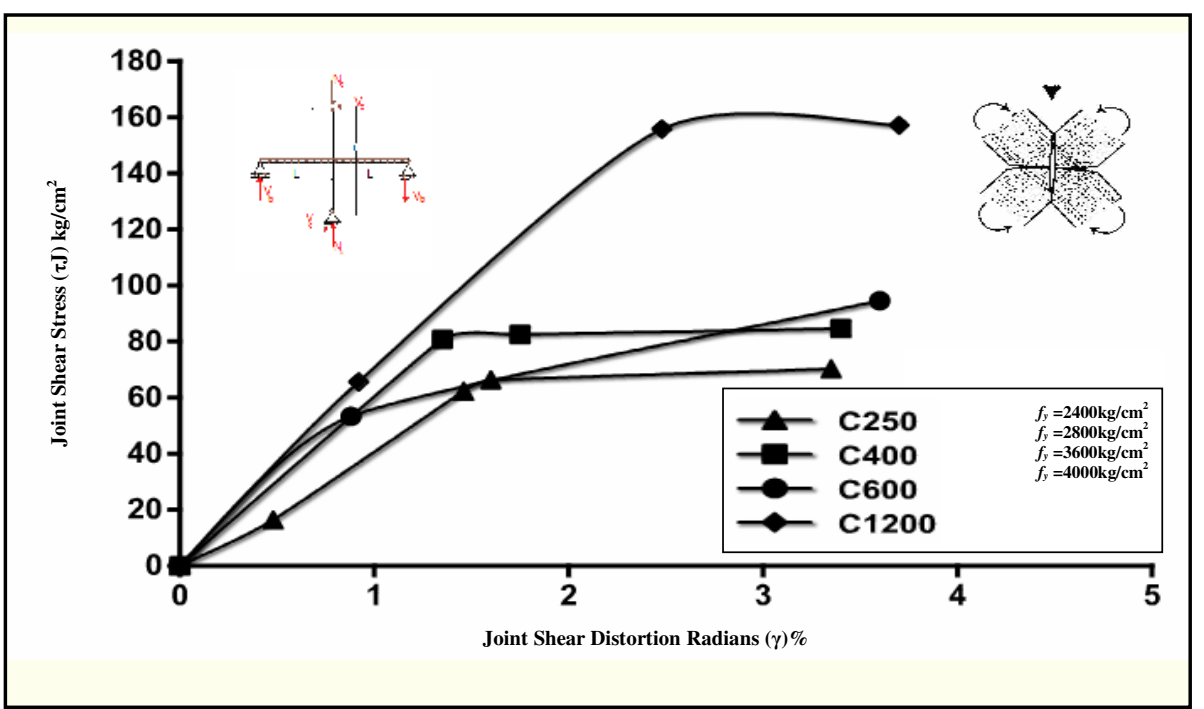

a) Relation of joint shear stress-joint shear strain joint (distortion) interior joint

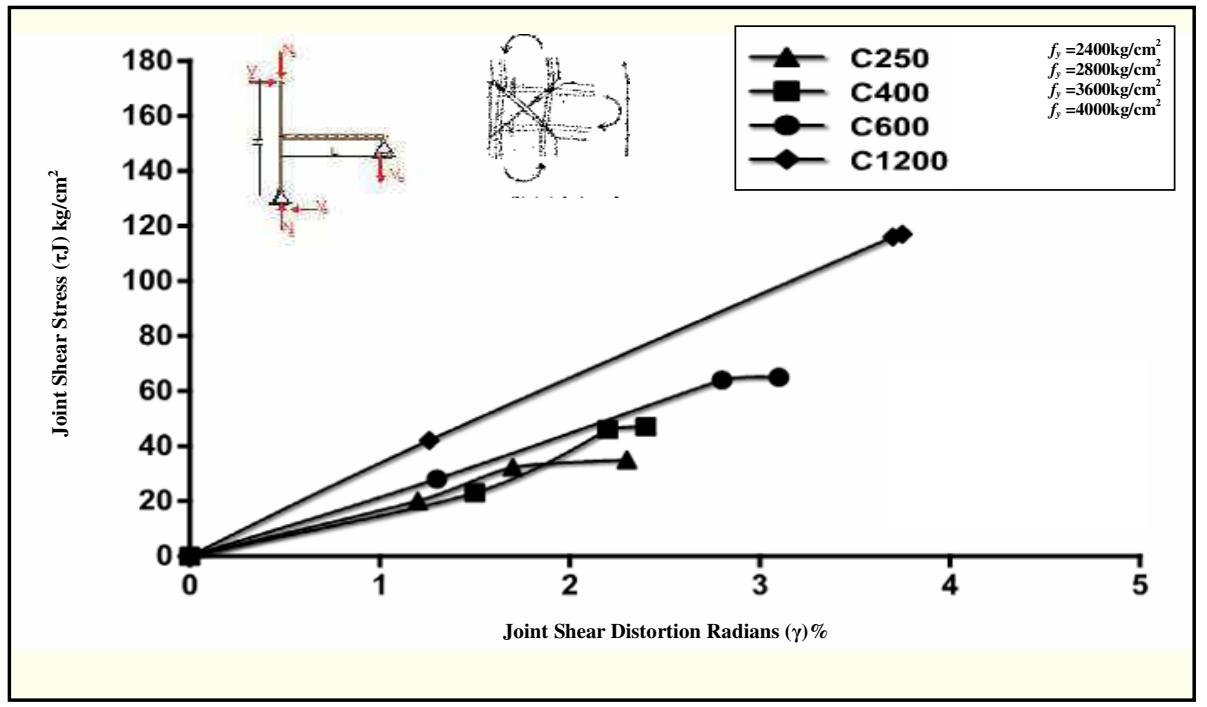

b) Relation of joint shear stress - (distortion) exterior joint

Journal of Engineering Sciences, Assiut University, Faculty of Engineering, Vol. 41, No. 3, May, 2013,E-mail address: jes@aun.edu.eg 
L. K. Idriss et al., Static behaviour of different types of R.C beam-column connections as affected by both value acting lateral horizontal force and grade of used concrete (theoretical study) part two, pp.746 - 814.

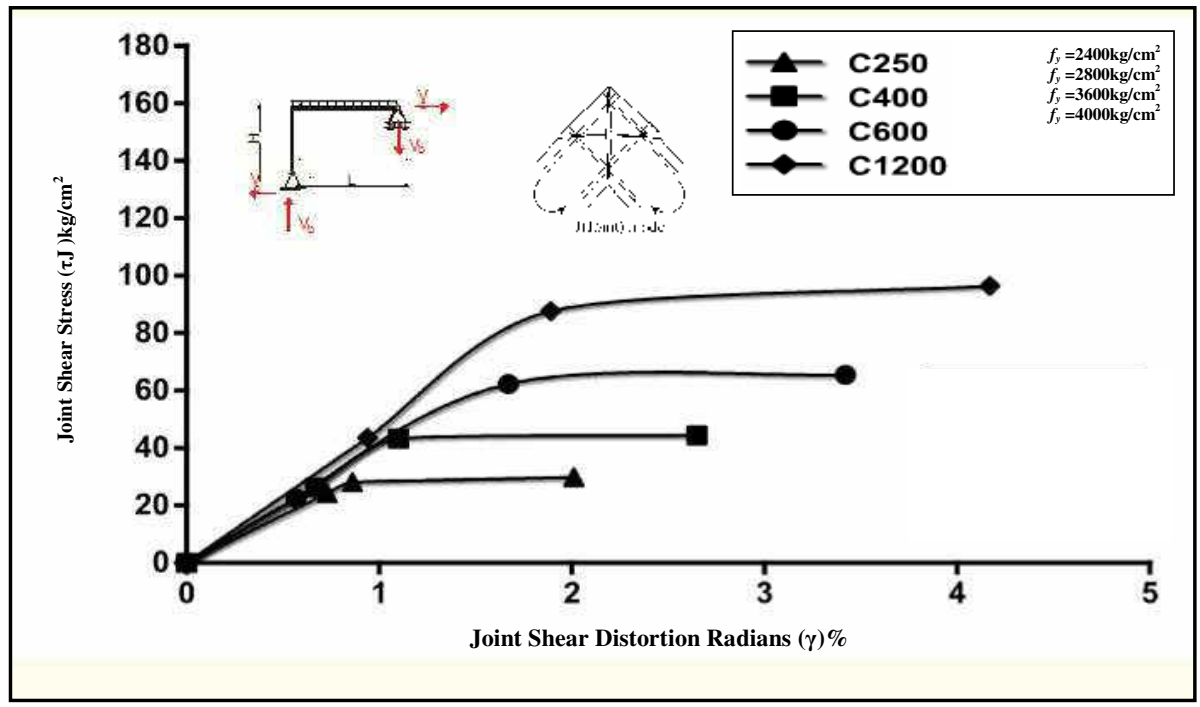

c) Relation of joint shear stress-joint (distortion) interior joint (distortion) corner joint

Fig. 14. Relation of Joint Shear Stress $\left(\tau_{\mathrm{j}}\right)-$ Joint Shear Strain $(\gamma \%)$ for Beam-Column Joints.

Fig. 14 illustrates that in general as the joint shear stress $\left(\tau_{\mathrm{j}}\right)$ increases, the corresponding joint shear strain $(\gamma)$ increases.

5. Relation of Principal Tensile Strain $\left(\varepsilon_{1}\right)$-Joint Shear Strain (Distortion) $(\gamma \%)$ radian.

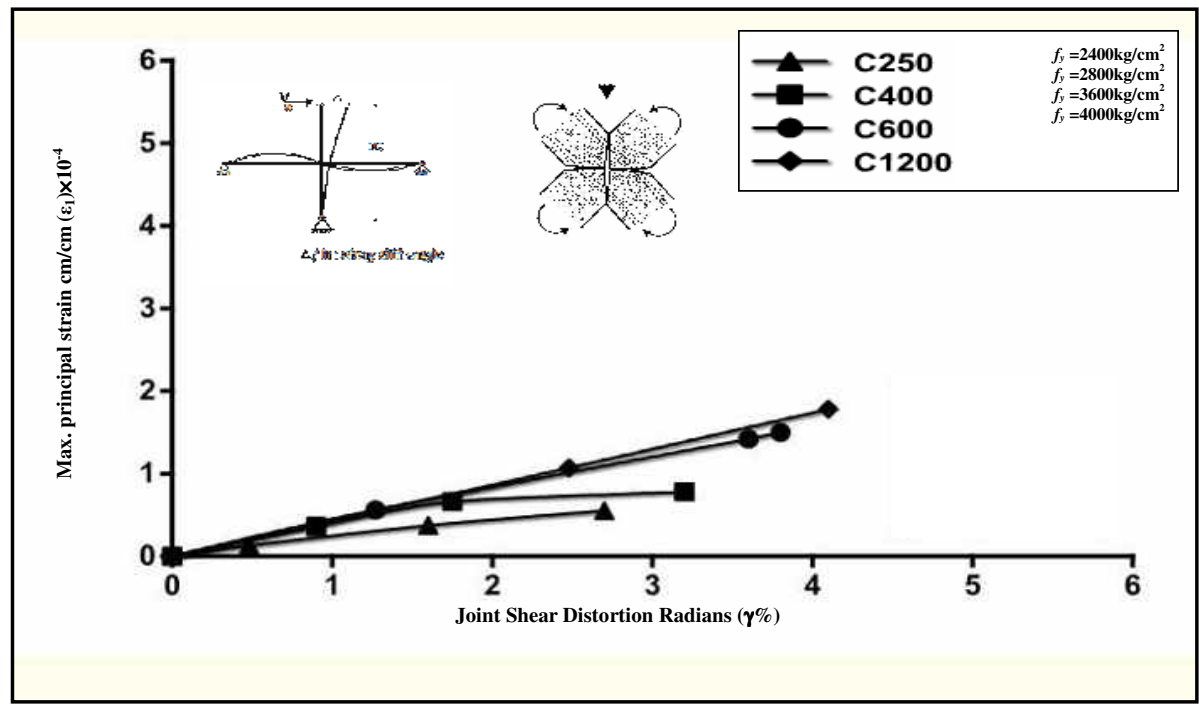

a) Relation of principal tensile strain-joint shear (distortion) interior strain joint

Journal of Engineering Sciences, Assiut University, Faculty of Engineering, Vol. 41, No. 3, May, 2013,E-mail address: jes@aun.edu.eg 
L. K. Idriss et al., Static behaviour of different types of R.C beam-column connections as affected by both value acting lateral horizontal force and grade of used concrete (theoretical study) part two, pp.746 - 814

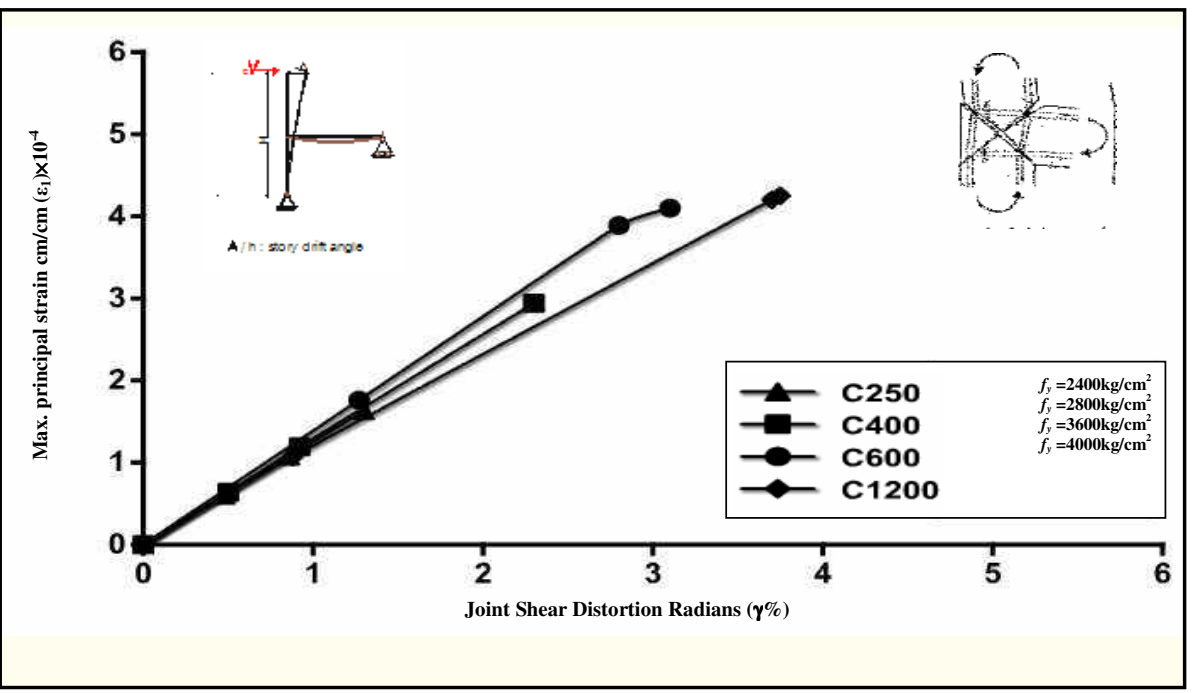

b) Relation of principal tensile strain-joint shear strain (distortion) exterior joint

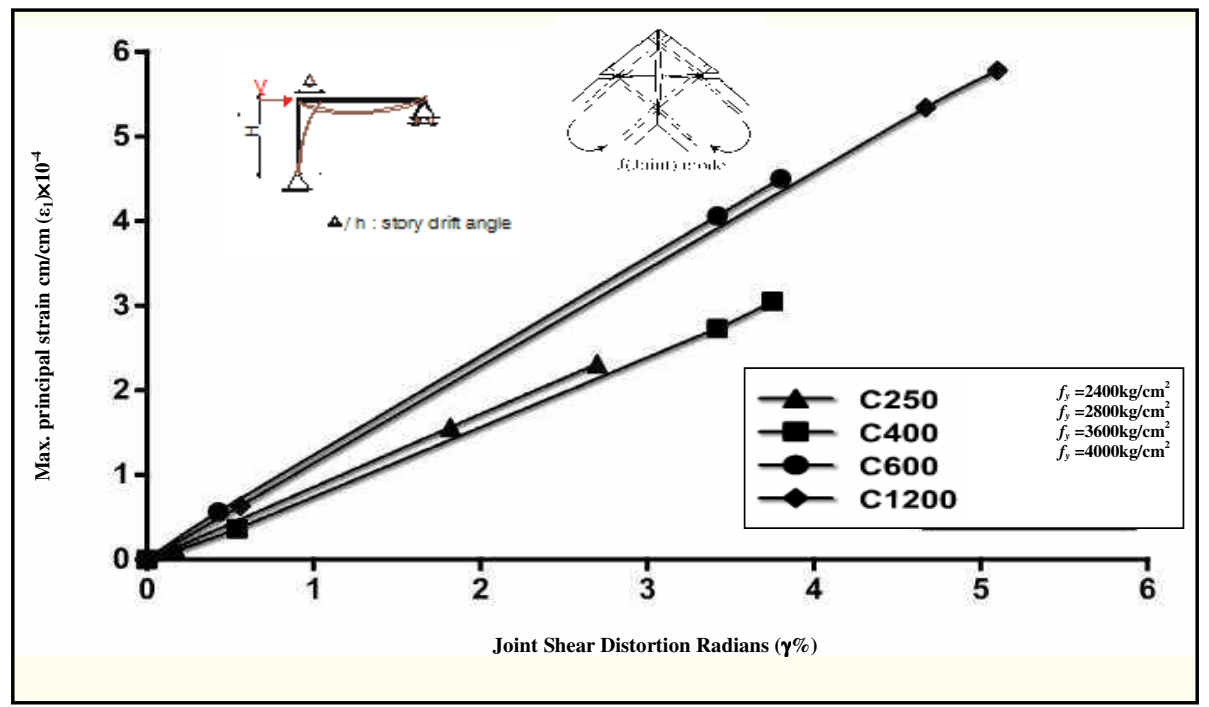

c) Relation of principal tensile strain-joint shear strain (distortion) corner joint

Fig. 15. Relation of Principal Tensile Strain $\left(\varepsilon_{1}\right)$ - Joint Shear Strain $(\gamma \%)$ for Beam-Column Joints

In terms of principal tensile strains, a nearly linear relationship with respect to the joint shear distortion was obtained for various levels as shown in Fig.15.

Journal of Engineering Sciences, Assiut University, Faculty of Engineering, Vol. 41, No. 3, May, 2013,E-mail address: jes@aun.edu.eg 
774

L. K. Idriss et al., Static behaviour of different types of R.C beam-column connections as affected by both value acting lateral horizontal force and grade of used concrete (theoretical study) part two, pp.746 - 814.

6. Relation of Principal Strain $\left(\varepsilon_{1}\right)-J o i n t$ Bond Stress $\left(\mu_{B}\right) \mathrm{kg} / \mathrm{cm}^{2}$.

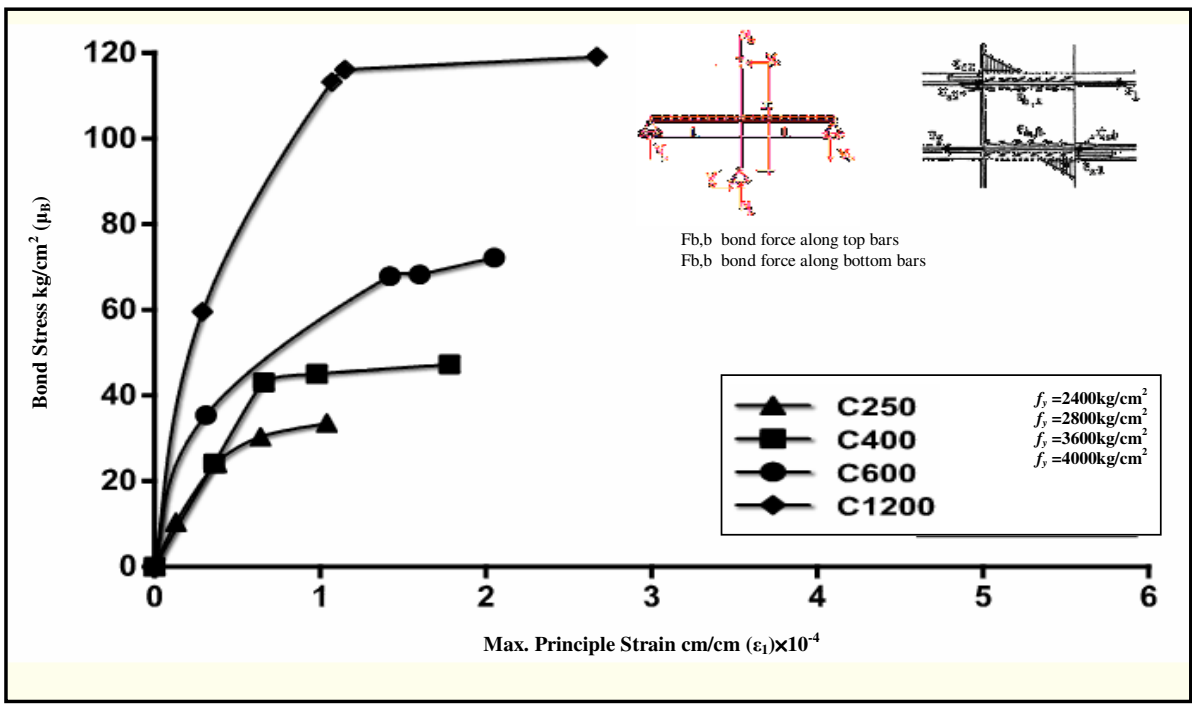

a) Relation of principal strain-joint bond stress for interior joint.

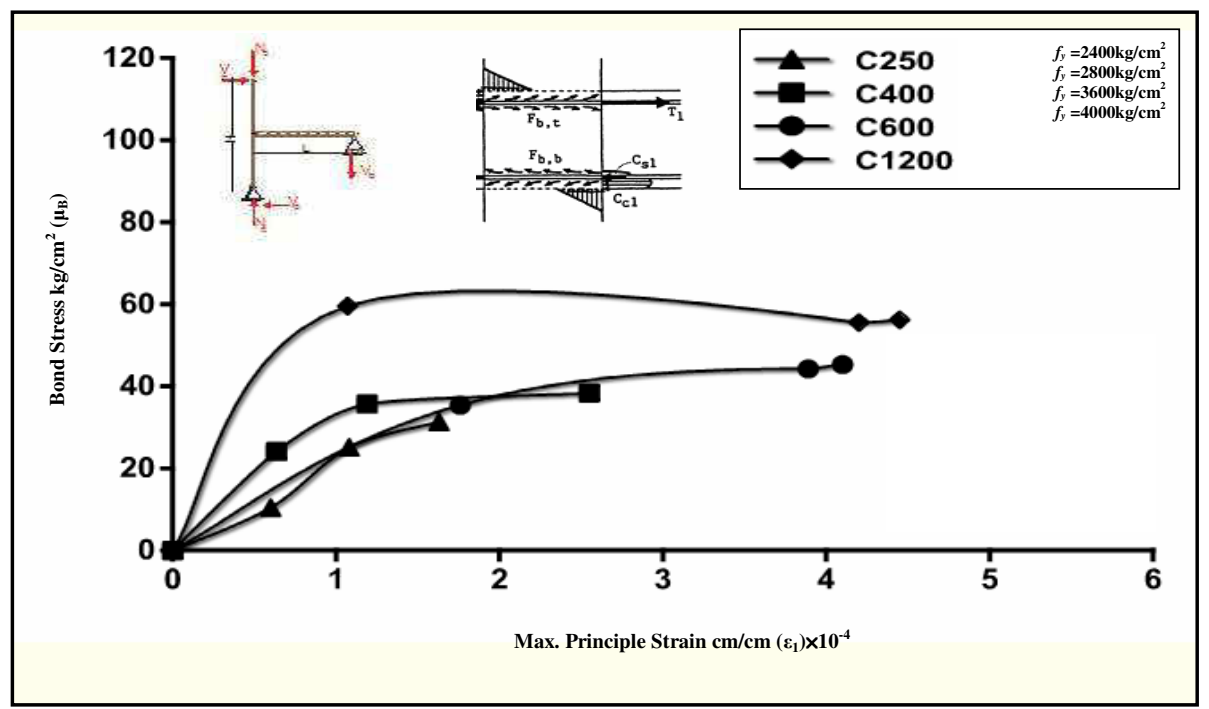

b) Relation of principal strain-joint bond stress for exterior joint

Journal of Engineering Sciences, Assiut University, Faculty of Engineering, Vol. 41, No. 3, May, 2013,E-mail address: jes@aun.edu.eg 
L. K. Idriss et al., Static behaviour of different types of R.C beam-column connections as affected by both value acting lateral horizontal force and grade of used concrete (theoretical study) part two, pp.746 - 814

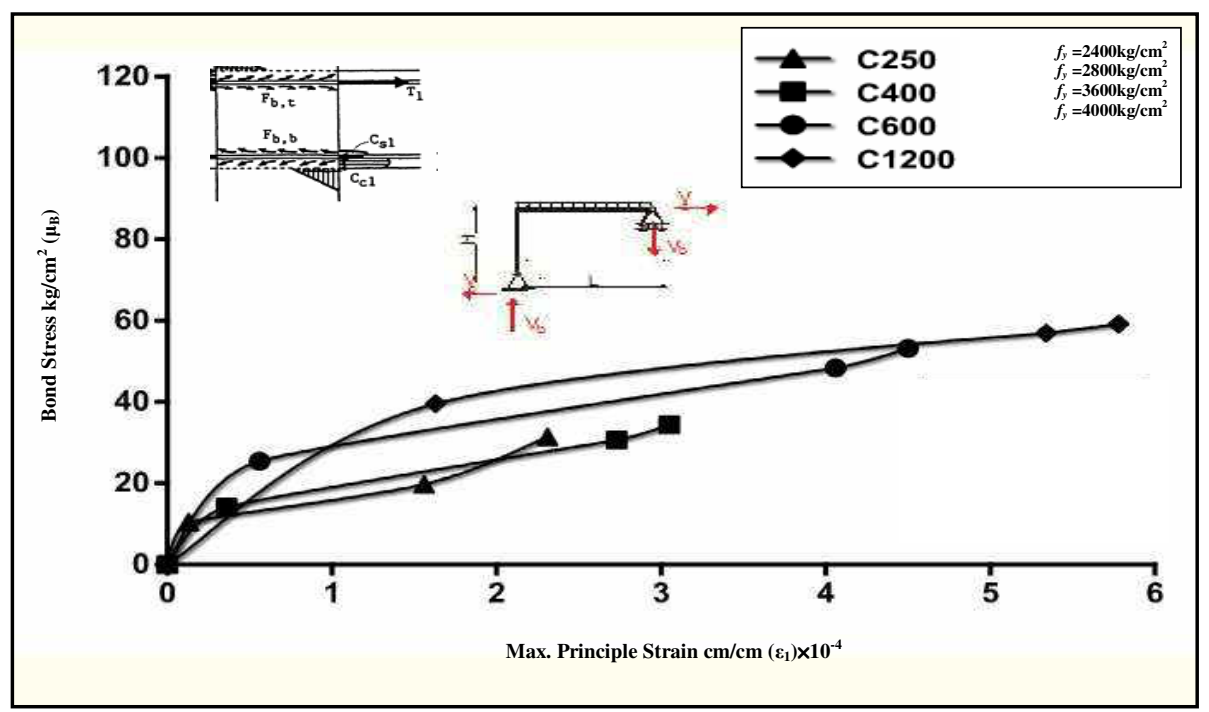

c) Relation of principal strain-joint bond stress for corner joint

Fig. 16. Relation of Principal Strain $\left(\varepsilon_{1}\right)$ - Joint Bond Stress $\left(\mu_{B}\right)$ for Joints

It can be seen in Fig. 16 that generally as the principal strains $\left(\varepsilon_{1}\right)$ increases, the corresponding joint bond stress $\left(\mu_{\mathrm{B}}\right)$ increases,

7. Relation of Joint Shear Stress $\left(\tau_{\mathrm{J}}\right) \mathrm{kg} / \mathrm{cm}^{2}$-Principle Axial Stress $\left(\sigma_{1}\right) \mathrm{kg} / \mathrm{cm}^{2}$.

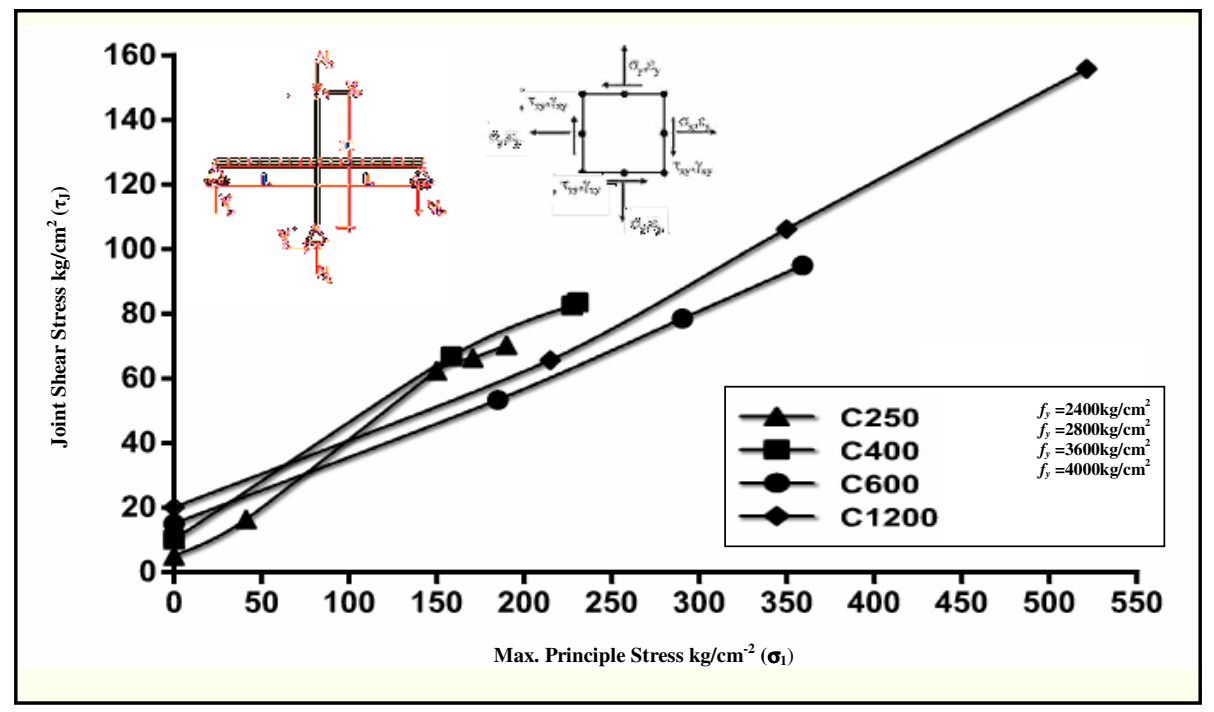

a) Relation of shear strength $\left(\tau_{\mathrm{J}}\right)$-axial stress $\left(\sigma_{1}\right)$ for interior

Journal of Engineering Sciences, Assiut University, Faculty of Engineering, Vol. 41, No. 3, May, 2013,E-mail address: jes@aun.edu.eg 
L. K. Idriss et al., Static behaviour of different types of R.C beam-column connections as affected by both value acting lateral horizontal force and grade of used concrete (theoretical study) part two, pp.746 - 814.

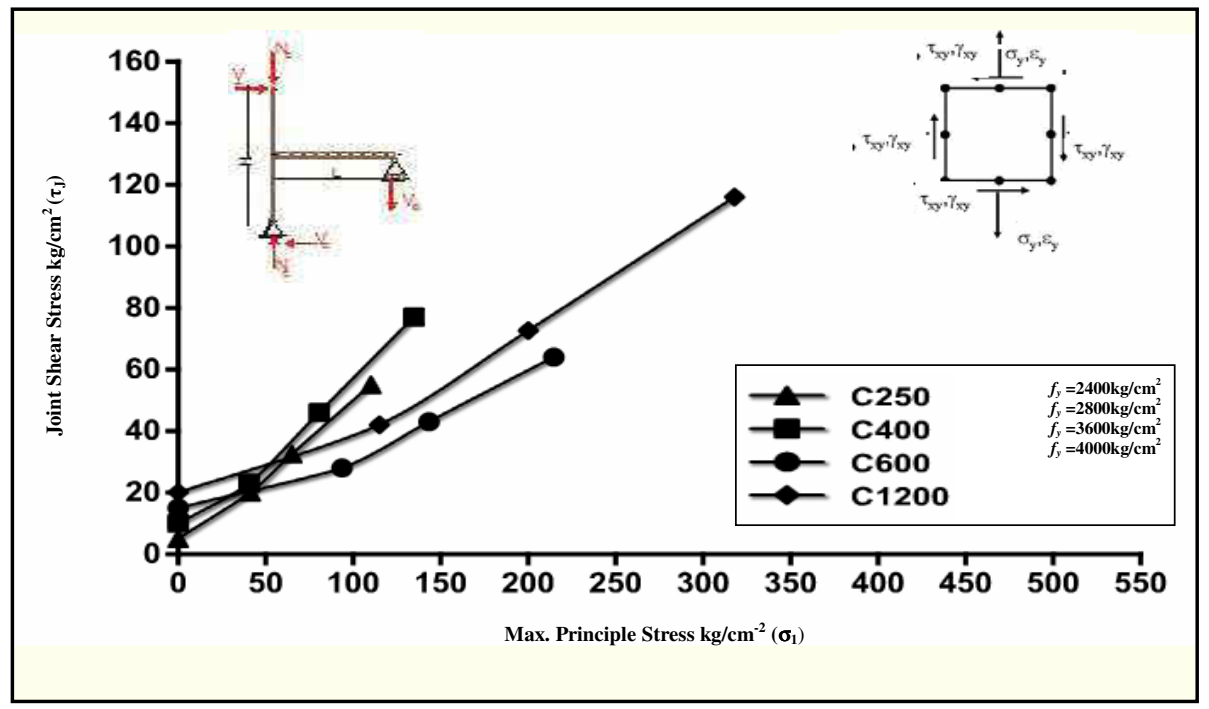

b) Relation of shear strength $\left(\tau_{\mathrm{J}}\right)$ - axial stress $\left(\sigma_{1}\right)$ for exterior joint

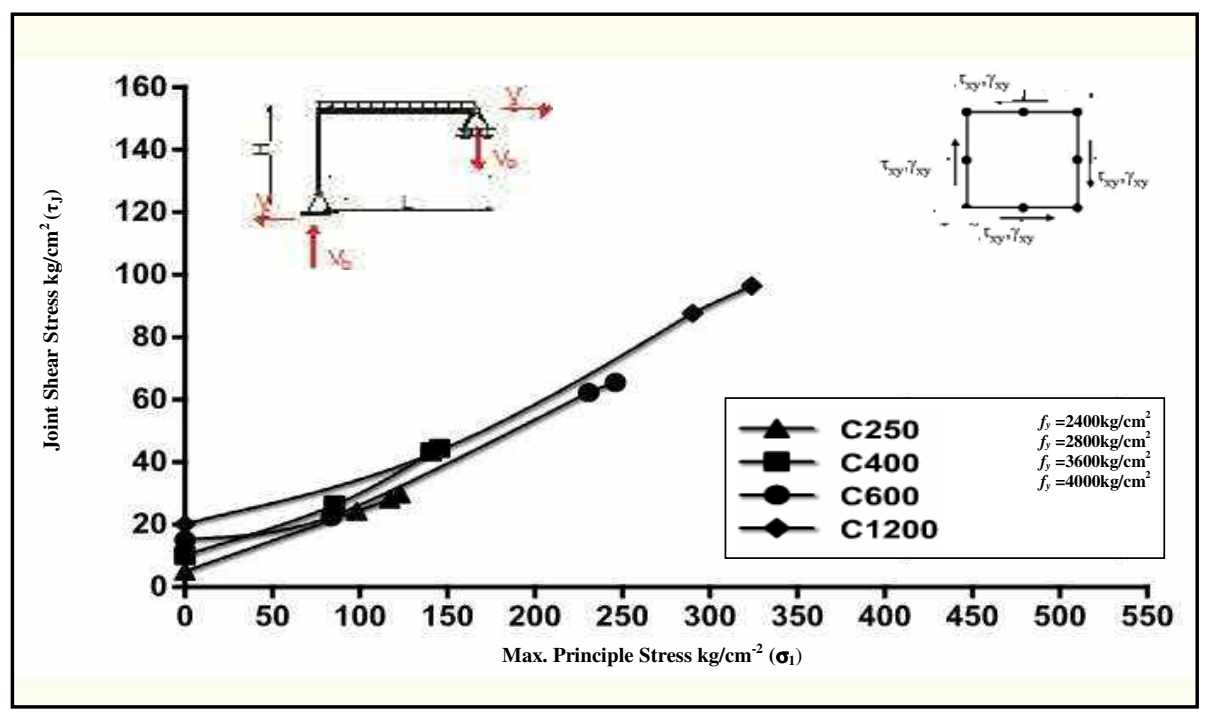

c) Relation of shear strength $\left(\tau_{\mathrm{J}}\right)$ - axial stress $\left(\sigma_{1}\right)$ for corner joint

Fig. 17. Relation of Joint Shear Strength $\left(\tau_{\mathrm{J}}\right)$ - Principal Axial Stress $\left(\sigma_{1}\right)$ for Joints

Journal of Engineering Sciences, Assiut University, Faculty of Engineering, Vol. 41, No. 3, May, 2013,E-mail address: jes@aun.edu.eg 
L. K. Idriss et al., Static behaviour of different types of R.C beam-column connections as affected by both value acting lateral horizontal force and grade of used concrete (theoretical study) part two, pp.746 - 814

Fig.17 illustrates that in general as the axial stress $\left(\sigma_{1}\right)$ increases the corresponding shear stress $\left(\tau_{\mathrm{J}}\right)$ increases too up to the maximum stresses.

\section{Relation of Principle Axial Stress $\left(\sigma_{1}\right)$-Principle Axial Strain( $\left(\varepsilon_{1}\right)$.}

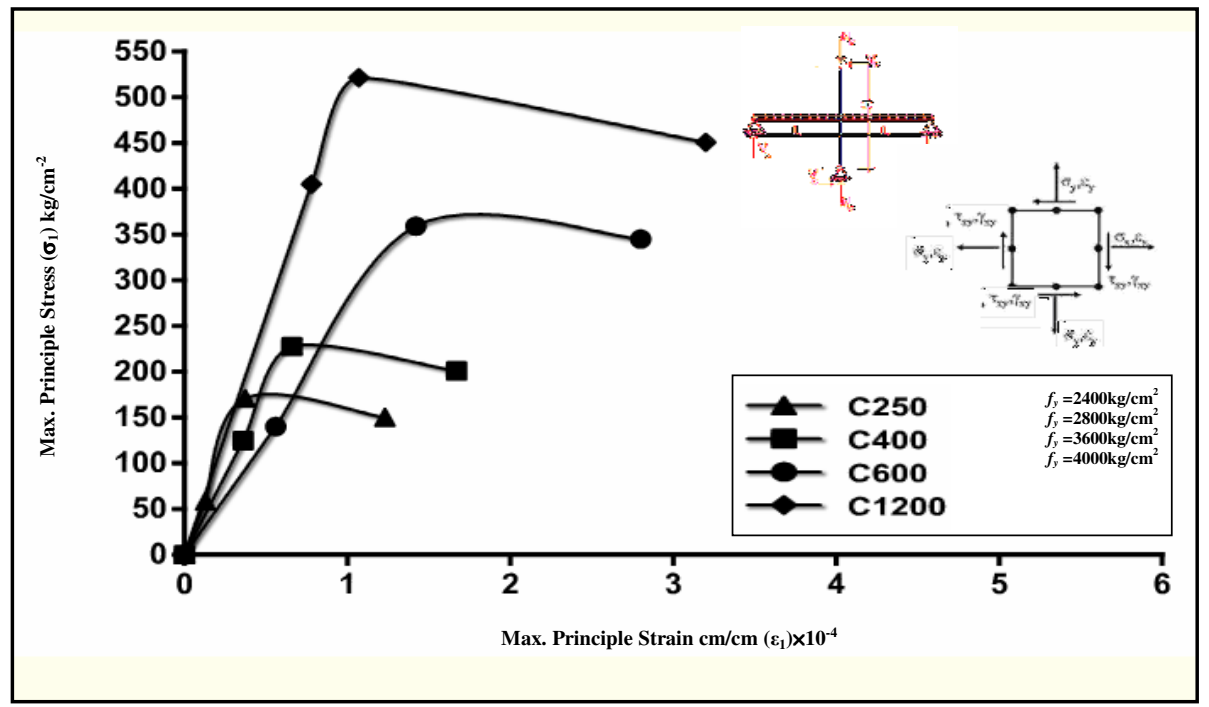

a) Relation of max. principle stress - max. principle strain for interior joint

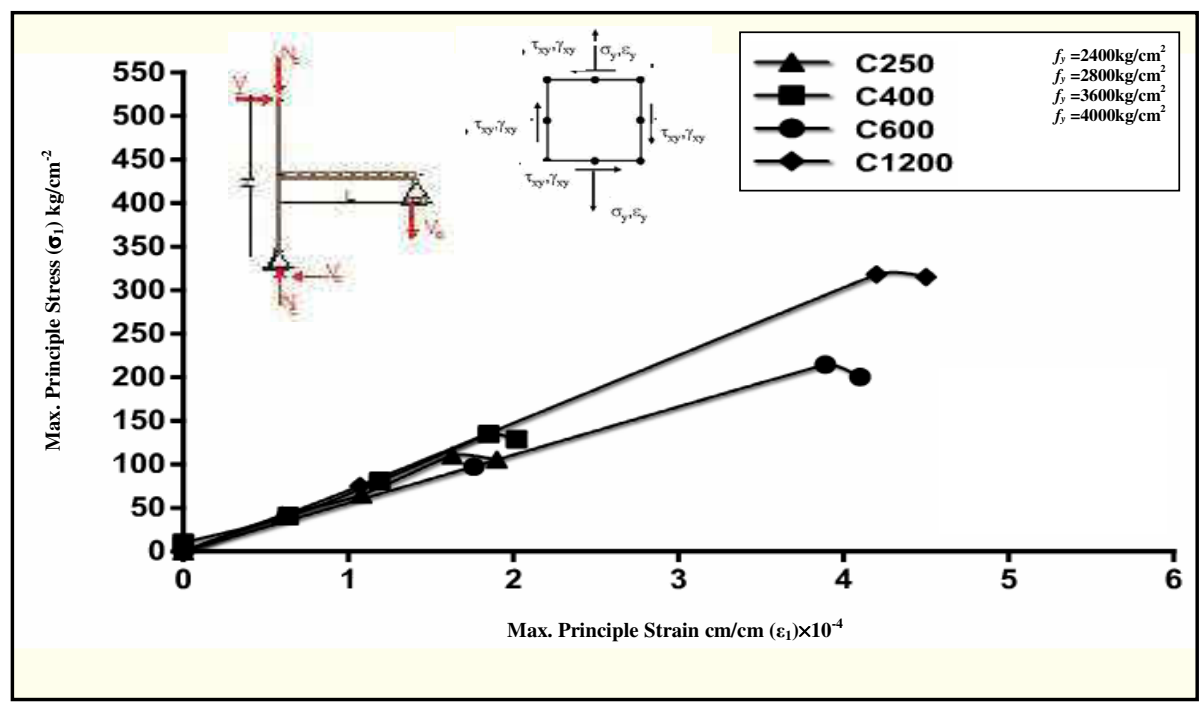

b) Relation of max. principle stress - max. principle strain for exterior joint

Journal of Engineering Sciences, Assiut University, Faculty of Engineering, Vol. 41, No. 3, May, 2013,E-mail address: jes@aun.edu.eg 
L. K. Idriss et al., Static behaviour of different types of R.C beam-column connections as affected by both value acting lateral horizontal force and grade of used concrete (theoretical study) part two, pp.746 - 814.

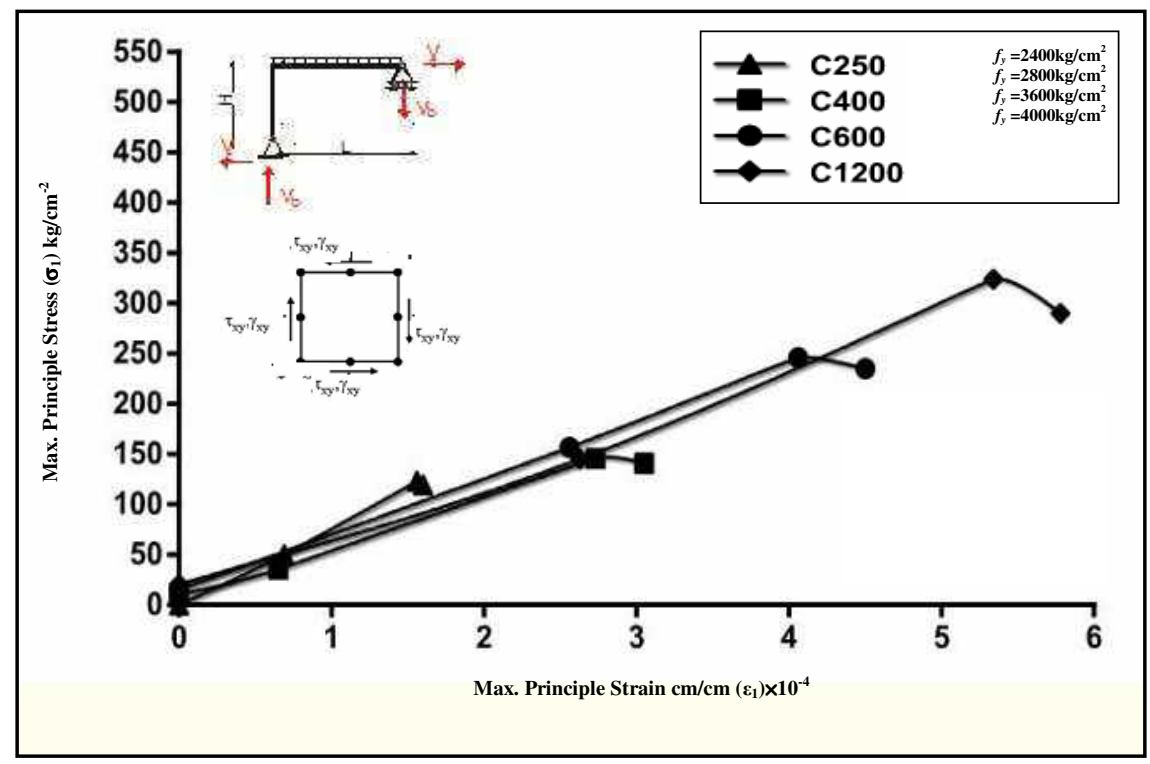

c) Relation of max. principle Stress - max. principle strain for corner joint

Fig.18. Relation of Maximum Principle Stress $\left(\sigma_{1}\right)$ - Maximum Principle Strain $\left(\varepsilon_{1}\right)$ for Beam-Column Joints

Generally, as the axial stress $\left(\sigma_{1}\right)$ increases corresponding axial strain $\left(\varepsilon_{1}\right)$ increases too up to the maximum stress as shown in Fig. 18

\section{Relation of Energy Absorption (EA)-Lateral Displacement( $\delta \mathrm{h})$}

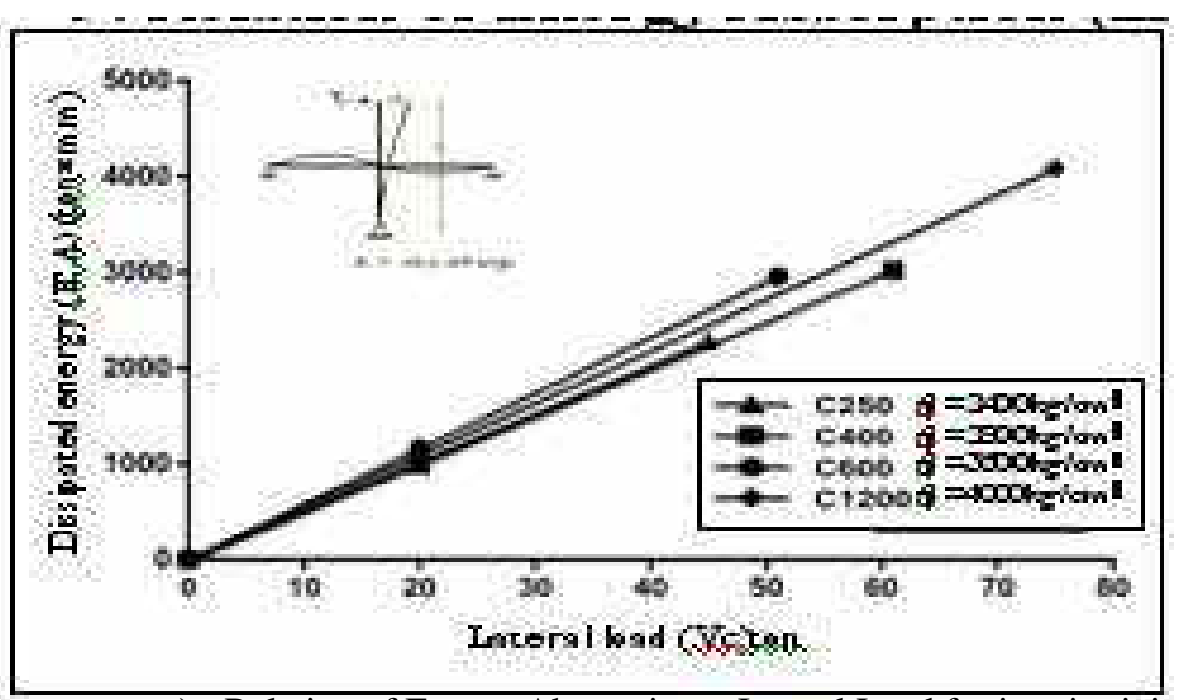

a) Relation of Energy Absorption - Lateral Load for interior joint

Journal of Engineering Sciences, Assiut University, Faculty of Engineering, Vol. 41, No. 3, May, 2013,E-mail address: jes@aun.edu.eg 
L. K. Idriss et al., Static behaviour of different types of R.C beam-column connections as affected by both value acting lateral horizontal force and grade of used concrete (theoretical study) part two, pp.746 - 814

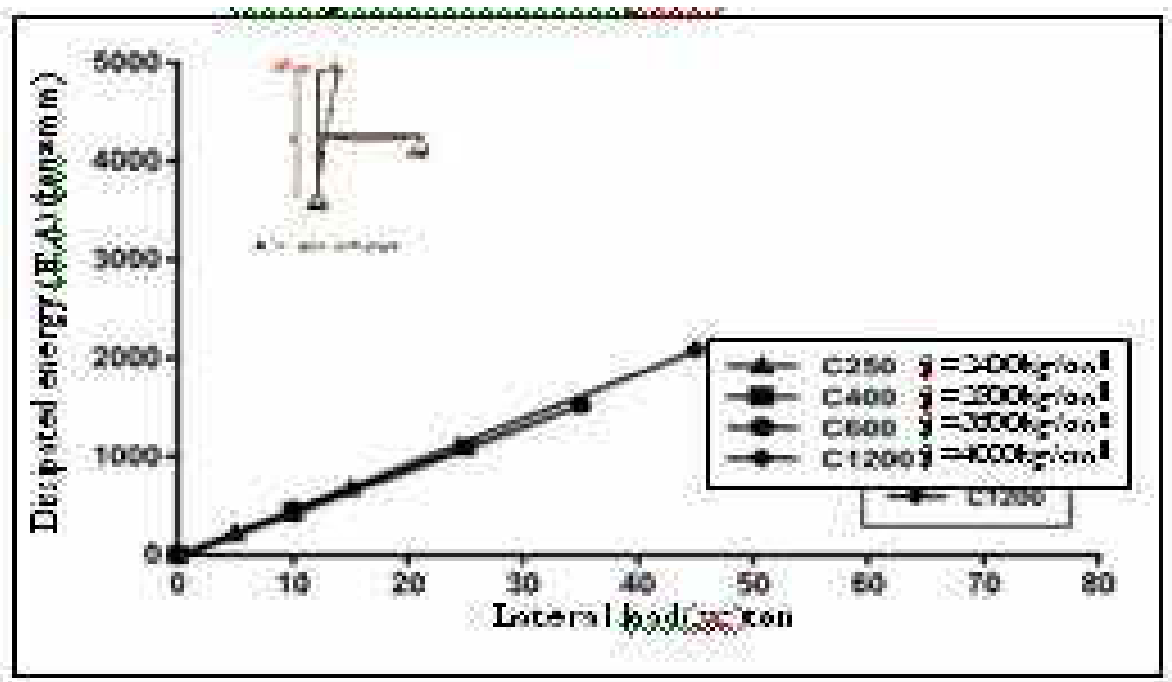

b) Relation of Energy Absorption - Lateral load for exterior joint

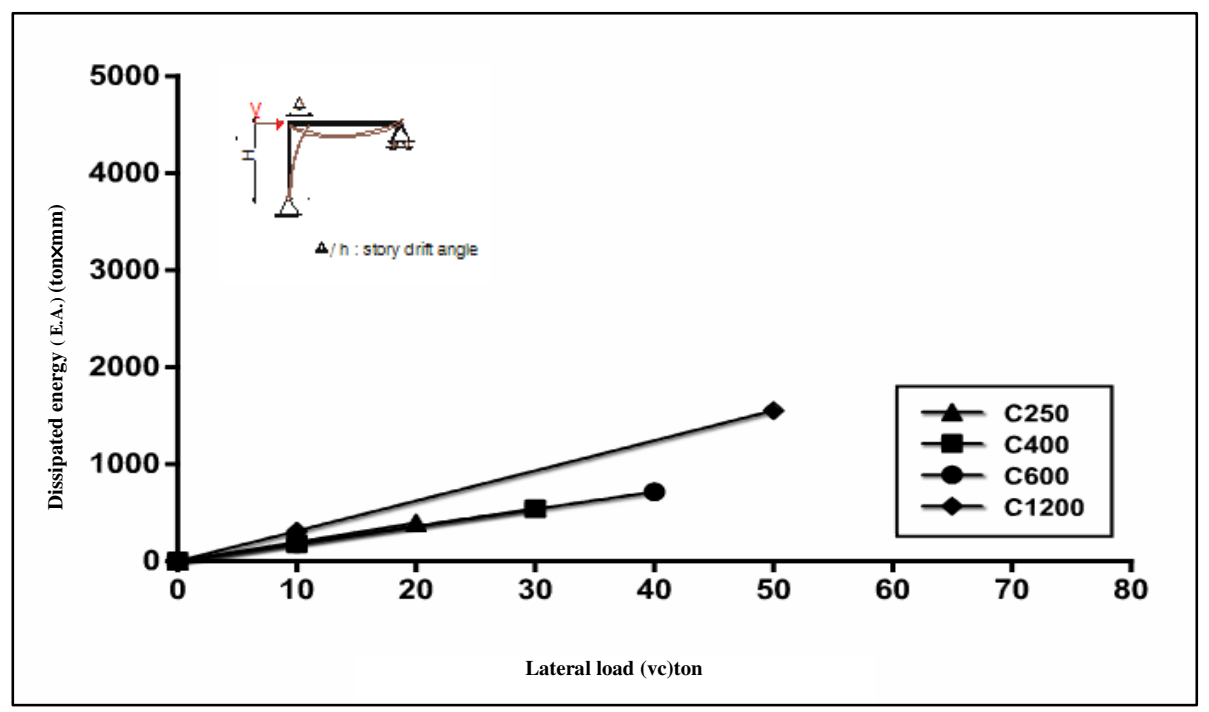

c) Relation of Energy Absorption (E.A.) (ton $\times \mathrm{mm}$ ) -Lateral Load (vc)ton for corner (Knee joint)

Fig.19. The Relation of Energy Absorption (E.A.) - Lateral Load(Vc)for Joints

a nearly linear relationship with respect to the lateral load was obtained for various levels energy absorption as shown in Fig.19.

Journal of Engineering Sciences, Assiut University, Faculty of Engineering, Vol. 41, No. 3, May, 2013,E-mail address: jes@aun.edu.eg 
780

L. K. Idriss et al., Static behaviour of different types of R.C beam-column connections as affected by both value acting lateral horizontal force and grade of used concrete (theoretical study) part two, pp.746 - 814 .

\section{Analysis and discussion of the obtained results}

\subsection{W.R.T Strength and Stresses Points of View:}

\subsubsection{Effect of Concrete Compressive Strength ( $\left.f^{\prime} c\right)$ on Joint Shear Stresses ( $\left.\tau J\right)$ :}

The obtained results on interior, exterior and knee connections indicated that the governing factor influencing the joint shear strength is the concrete compressive strength

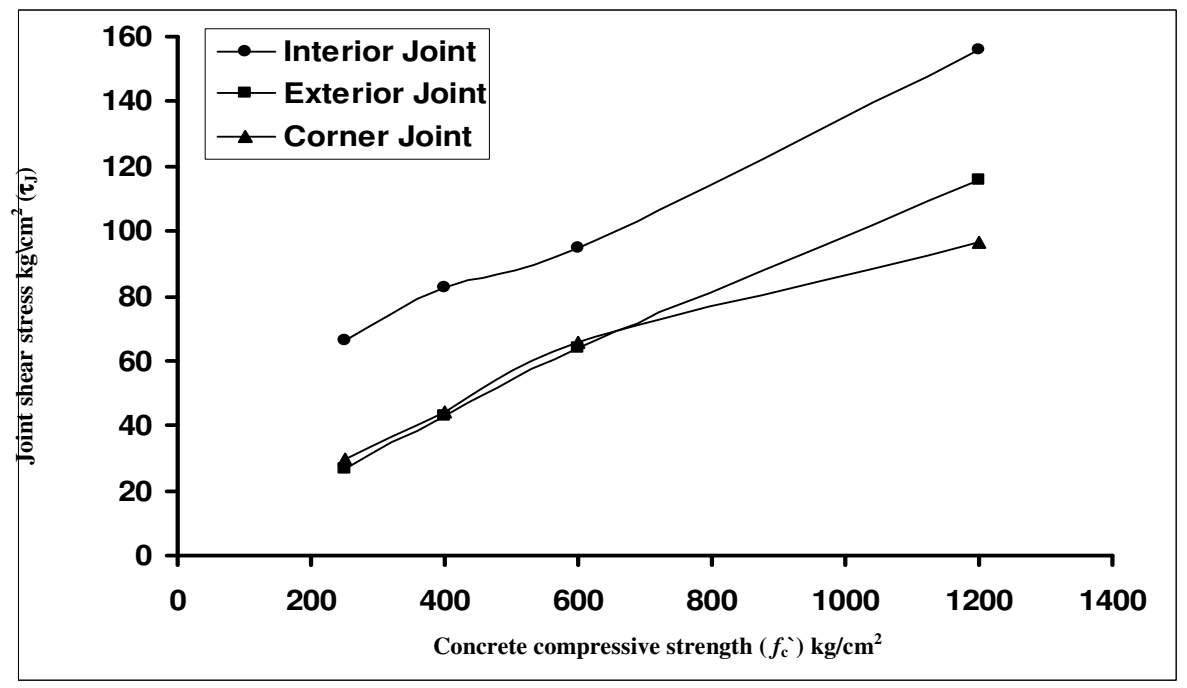

Fig.20. Relation of Concrete Compressive Strength (f'c) - Joint Shear Stress $(\tau j)$

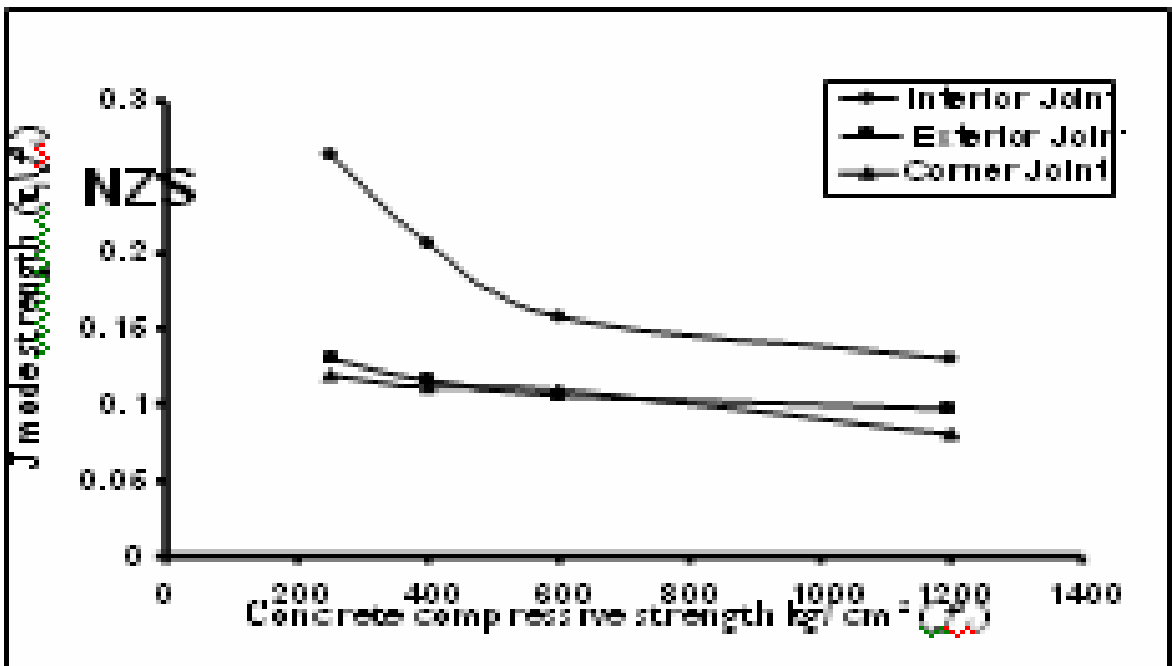

Fig. 21. Relation of Concrete Compressive Strength $\left(\dot{f}_{\mathrm{c}}\right)$ - Joint Mode Strength (Joint Shear-Resistance)

Journal of Engineering Sciences, Assiut University, Faculty of Engineering, Vol. 41, No. 3, May, 2013,E-mail address: jes@aun.edu.eg 
L. K. Idriss et al., Static behaviour of different types of R.C beam-column connections as affected by both value acting lateral horizontal force and grade of used concrete (theoretical study) part two, pp.746 - 814

Based on Table 5 and Figures 20 and 21, it is obvious that; as the compressive strength of concrete increases the corresponding joint shear stress increases for all types of joints. Also it obvious that the interior joints posses higher joint shear stress in comparison with both that for exterior or corner joint. Also Figure 20 indicates that use of high strength concrete has a beneficial effect on increasing the connection shear strength compared to that of normal-strength. An increase of about $2.5 \%$ for interior joint, $4.8 \%$ for exterior joint and 3.5\% for knee joint were observed. The results were compared with that obtained by (Felica th. 2007) studies, where an increase of about $5 \%$ case of no moment was stated. The $(\mathrm{J})$ mode strength, defined by $\left(\tau \mathrm{j} / f_{\mathrm{c}}^{\prime}\right)$, for the various joints was calculated and plotted against the corresponding compressive strength $\left(f_{\mathrm{c}}\right)$ as shown in Figure 21. Figure 21 declared that the $\left(\tau \mathrm{j} / f_{\mathrm{c}}\right)$ mode strength decrease by increase the grade of concrete. Higher values were corresponding to interior joints rather than that for exterior joints and finally smaller value were for corner joints.

Table 6 gives the available codes recommendations for joint shear strength values for the studied types of R.C joints. Table 7 also indicates a comparison between the calculated values of joint shear strength as reported in the commentary of different Codes and the obtained theoretical results of beam-column joints in our study.

The obtained values of joint shear stress $\left(\tau_{\mathrm{j}}\right)$ as calculated by the available Codes as well as the our obtained results are plotted against the used corresponding concrete strength $\left(\dot{f}_{\mathrm{c}}\right)$ in Figures 22, 23 and 24 for the studied joints.

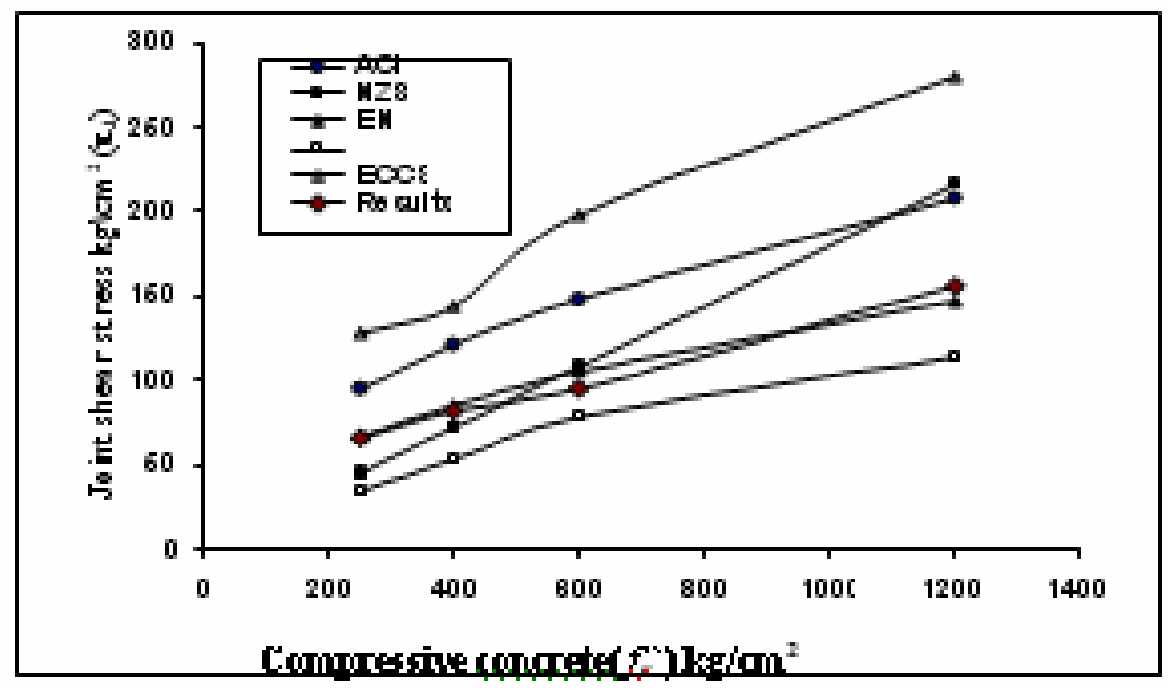

Fig. 22. Concrete Compressive Strength $\left(\dot{f}_{\mathrm{c}}\right)-$ Joint Shear Stress $\left(\tau_{\mathrm{j}}\right)$ Interior Joint

Journal of Engineering Sciences, Assiut University, Faculty of Engineering, Vol. 41, No. 3, May, 2013,E-mail address: jes@aun.edu.eg 
L. K. Idriss et al., Static behaviour of different types of R.C beam-column connections as affected by both value acting lateral horizontal force and grade of used concrete (theoretical study) part two, pp.746 - 814.

\section{Table 5.}

Effect of Concrete Compressive Strength on Shear Stresses on Beam- Column Joint.

\begin{tabular}{|c|c|c|c|c|c|c|c|c|c|c|}
\hline & Joint No. & $\begin{array}{c}\text { Yield } \\
\text { strength } \\
\left(f_{y}\right) \mathrm{kg} / \mathrm{cm}^{2}\end{array}$ & $\begin{array}{c}\mathbf{N}_{c} / \\
\left(f_{c}\right) \mathbf{A}_{c}\end{array}$ & $\begin{array}{l}\text { Lateral } \\
\text { load ton } \\
\left(V_{c}\right)\end{array}$ & $\begin{array}{l}\text { Joint shear } \\
\text { stress } \\
\mathrm{kg} / \mathrm{cm}^{2}\left(\tau_{\mathrm{J}}\right)\end{array}$ & $\begin{array}{c}\text { Max principal } \\
\text { stress } \\
\mathrm{kg} / \mathrm{cm}^{2}\left(\sigma_{1}\right)\end{array}$ & $\begin{array}{l}\text { Joint bond } \\
\text { stress } \\
\mathrm{kg} / \mathrm{cm}^{2}\left(\mu_{\mathrm{b}}\right)\end{array}$ & $\begin{array}{l}(J) \text { mode } \\
\text { strength } \\
\left(\tau_{\mathrm{J}}\right) /\left(f_{\mathrm{c}}\right)\end{array}$ & $\begin{array}{c}\text { Energy } \\
\text { Absorption } \\
\text { (E.A) Ton } \times \mathbf{m m}\end{array}$ & $\begin{array}{c}\text { Max. principal } \\
\text { stress / } \\
\text { compressive } \\
\text { stress } \\
\left(\sigma_{1}\right) /\left(f c^{\prime}\right) \\
\end{array}$ \\
\hline \multirow{4}{*}{. } & $\mathrm{J}(4) \mathrm{C} 250$ & 2400 & 0.15 & 45 & 66.30 & 170.74 & 24.11 & 0.265 & 2278 & 0.68 \\
\hline & $\mathrm{J}(8) \mathrm{C} 400$ & 2800 & 0.15 & 51 & 82.50 & 227.50 & 43.02 & 0.521 & 2959 & 0.57 \\
\hline & $\mathrm{J}(12) \mathrm{C} 600$ & 3600 & 0.15 & 61 & 94.95 & 359.1 & 67.87 & 0.158 & 3020 & 0.59 \\
\hline & $\mathrm{J}(16) \mathrm{C} 1200$ & 4000 & 0.15 & 75 & 155.8 & 521.4 & 113.20 & 0.095 & 4083 & 0.45 \\
\hline \multirow{4}{*}{ 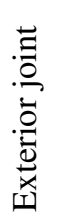 } & $\mathrm{J}(20) \mathrm{C} 250$ & 2400 & 0.15 & 15 & 32.50 & 65 & 25.20 & 0.202 & 690 & 0.26 \\
\hline & J (24)C400 & 2800 & 0.15 & 25 & 46.43 & 80.62 & 35.60 & 0.107 & 1098 & 0.20 \\
\hline & J (28)C600 & 3600 & 0.15 & 35 & 64 & 214.6 & 44.25 & 0.265 & 1520 & 0.35 \\
\hline & $\mathrm{J}(32 \mathrm{C} 1200$ & 4000 & 0.15 & 45 & 116 & 318 & 55.54 & 0.097 & 2082 & 0.26 \\
\hline \multirow{4}{*}{ 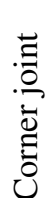 } & $\mathrm{J}(36) \mathrm{C} 250$ & 2400 & 0.15 & 20 & 29.77 & 122.95 & 19.69 & 0.12 & 395 & 0.49 \\
\hline & $\mathrm{J}(40) \mathrm{C} 400$ & 2800 & 0.15 & 30 & 44.30 & 145.50 & 30.59 & 0.11 & 540 & 0.36 \\
\hline & $\mathrm{J}(44) \mathrm{C} 600$ & 3600 & 0.15 & 40 & 65.47 & 245.9 & 48.33 & 0.109 & 715 & 0.41 \\
\hline & $\mathrm{J}(48) \mathrm{C} 1200$ & 4000 & 0.15 & 50 & 96.40 & 323.90 & 65.90 & 0.08 & 1552 & 0.27 \\
\hline
\end{tabular}


L. K. Idriss et al., Static behaviour of different types of R.C beam-column connections as affected by both value acting lateral horizontal force and grade of used concrete (theoretical study) part two, pp.746 - 814

Table 6.

Codes Recommendations for Shear Strength of Different Types of Joints.

\begin{tabular}{|c|c|c|c|}
\hline $\begin{array}{l}\text { Different } \\
\text { Codes }\end{array}$ & $\begin{array}{c}\text { Nominal Joint Shear } \\
\text { Strength (Interior Joint) }\end{array}$ & $\begin{array}{l}\text { Nominal Joint } \\
\text { Shear Strength } \\
\text { (Exterior Joint ) }\end{array}$ & $\begin{array}{l}\text { Nominal Joint } \\
\text { Shear Strength } \\
\text { (Knee Joint) }\end{array}$ \\
\hline $\begin{array}{l}\text { ACI 352R-02 } \\
\text { and ACI 318-02 }\end{array}$ & $V_{\mathrm{n}}=\gamma_{\mathrm{ACl}} \sqrt{f_{\mathrm{c}}^{\prime}} \mathrm{b}_{\mathrm{j}} \mathrm{h}_{\mathrm{c}}$ & $\begin{array}{l}\text { Vn ex. }=0.75 \\
\text { Vn int. }\end{array}$ & 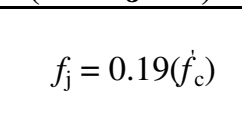 \\
\hline ECCS 203 (2001) & $\tau_{\mathrm{j}}=0.96 A_{\mathrm{j}} \sqrt{f_{\mathrm{c}}^{\prime}}$ & & \\
\hline AIJ 1997 & $\begin{aligned} V_{\mathrm{j}} & =\mathrm{k} \phi f_{\mathrm{j}} \mathrm{b}_{\mathrm{j}} \mathrm{D}_{\mathrm{j}}, \text { or } \\
\mathrm{vj}_{\mathrm{j}} & =0.8 \times\left(\dot{f}_{\mathrm{c}}\right)^{0.7}\end{aligned}$ & $\begin{array}{l}f_{\mathrm{j}} \text { ex. }=0.9 f_{\mathrm{j}} \\
\text { int. }\end{array}$ & ICI \\
\hline NZS 3101: 1995 & $f_{\mathrm{j}}=0.2 \times\left(\dot{f}_{\mathrm{c}}\right) \mathrm{Aj}$ & $\begin{array}{c}f_{\mathrm{j}} \text { ex. }=0.98 f_{\mathrm{j}} \\
\text { int. }\end{array}$ & \\
\hline EN 1998-1: 2003 & $V_{\mathrm{jh}} \leq \eta f_{\mathrm{cd}} \sqrt{1-\frac{V_{\mathrm{d}}}{\eta}}$ & $\begin{array}{r}\mathrm{V}_{\mathrm{jh}} \text { Ex. }= \\
0.80 \mathrm{Vjh} \text { Int. }\end{array}$ & \\
\hline
\end{tabular}

\section{Remarks and Symbols:}

$V_{\mathrm{n}}$ : nominal shear strength of beam-column joints according to the general procedures of ACI $318 ; \boldsymbol{A}_{j}:$ is the effective horizontal joint area defined as the product of the column dimension in the direction of loading; $\boldsymbol{\gamma}_{\mathrm{ACI}}$ : is the joint shear strength factor $=0.67 ; \boldsymbol{f}^{\prime}$ : is the specified concrete compressive strength; $\mathbf{b}_{\mathbf{j}}$ : is the effective joint shear width; $\mathbf{h}_{\mathbf{c}}$ : is the column depth; $\boldsymbol{\tau}_{\mathbf{J}}$ : the nominal shear strength of the joint as a function of only concrete strength; $\mathbf{v}_{\mathbf{j h}}$ : nominal shear stress; $\mathbf{v}_{\mathrm{d}}$ : normalized axial force ratio on column; $\eta$ : denotes the reduction factor on concrete compressive strength due to tensile strains in transverse direction; $\boldsymbol{f}_{\text {cd }}$ : concrete cylinder compressive strength; $\mathbf{k}$ : is the factor dependent on the shape of in-plane geometry (1.0 for interior connections, 0.7 for exterior connections and T-shape top story joints. and 0.4 for corner knee connections); $\phi$ : is the factor for the effect of out-of-plane geometry (1.0 for joints with transverse beams on both sides and 0.85 for other types of joints); $f_{\mathrm{j}}$ : is the standard value of the joint shear strength (as a function of concrete compressive strength); and $\boldsymbol{D}_{\mathbf{j}}$ : is the effective column depth. ;Int.: internal joint, 
784

L. K. Idriss et al., Static behaviour of different types of R.C beam-column connections as affected by both value acting lateral horizontal force and grade of used concrete (theoretical study) part two, pp.746 - 814.

\section{Table 7.}

The Calculated Values of the Available Codes Recommendations as well as Obtained Results for Joint Shear Strength $\left(\tau_{\mathrm{J}}\right)$ for the Studied Joints.

\begin{tabular}{|c|c|c|c|c|c|c|c|c|}
\hline \multicolumn{2}{|r|}{ Joint No } & $\begin{array}{c}\text { Yield } \\
\text { strength } \\
\left(f_{y}\right) \\
\left(\mathrm{kg} / \mathrm{cm}^{2}\right)\end{array}$ & $\begin{array}{c}\text { Experimental } \\
\text { results } \\
\left(\tau_{\mathrm{J}}\right)\left(\mathrm{kg} / \mathrm{cm}^{2}\right)\end{array}$ & $\begin{array}{c}\text { ACI 352R-02 and } \\
\operatorname{ACI~318-02}\left(\tau_{\mathrm{J}}\right) \\
\left(\mathrm{kg} / \mathrm{cm}^{2}\right)\end{array}$ & $\begin{array}{c}\text { NZS 3101: } \\
1995 \\
\left(\tau_{J}\right)\left(\mathrm{kg} / \mathrm{cm}^{2}\right)\end{array}$ & $\begin{array}{l}\text { EN 1998-1:2003 } \\
\left(\tau_{J}\right)\left(\mathrm{kg} / \mathrm{cm}^{2}\right)\end{array}$ & $\begin{array}{c}\text { AIJ } 1997 \\
\left(\tau_{J}\right)\left(\mathrm{kg} / \mathrm{cm}^{2}\right)\end{array}$ & $\begin{array}{c}\text { ECCS } 203 \\
(2001) \\
\left(\tau_{J}\right)\left(\mathrm{kg} / \mathrm{cm}^{2}\right)\end{array}$ \\
\hline \multirow{4}{*}{ 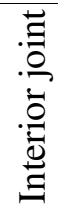 } & $\mathrm{J}(4) \mathrm{C} 250$ & 2400 & 66.30 & 95.34 & 45 & 67.45 & 34.34 & 128 \\
\hline & $\mathrm{J}(8) \mathrm{C} 400$ & 2800 & 82.50 & 120.60 & 72 & 85.20 & 53.03 & 144 \\
\hline & J (12)C600 & 3600 & 94.95 & 147.70 & 108 & 104.37 & 77.52 & 198 \\
\hline & $\mathrm{J}(16) \mathrm{C} 1200$ & 4000 & 155.8 & 208 & 216 & 147.37 & 114.42 & 280.59 \\
\hline \multirow{4}{*}{ 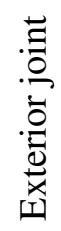 } & $\mathrm{J}(20) \mathrm{C} 250$ & 2400 & 32.5 & 71.50 & 44 & 27.47 & 30.90 & \\
\hline & $\mathrm{J}(24) \mathrm{C} 400$ & 2800 & 46.63 & 90.45 & 71 & 42.42 & 47.70 & \\
\hline & $\mathrm{J}(28) \mathrm{C} 600$ & 3600 & 64 & 110.7 & 105 & 62.01 & 69.76 & \\
\hline & $\mathrm{J}(32 \mathrm{C} 1200$ & 4000 & 116 & 157 & 211 & 117.6 & 102.6 & \\
\hline \multirow{3}{*}{ 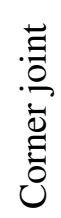 } & $\mathrm{J}(36) \mathrm{C} 250$ & 2400 & 29.77 & 47.50 & & & 23 & \\
\hline & $\begin{array}{l}\mathrm{J}(40) \mathrm{C} 400 \\
\mathrm{~J}(44) \mathrm{C} 600\end{array}$ & $\begin{array}{l}2800 \\
3600\end{array}$ & $\begin{array}{l}44.30 \\
65.47\end{array}$ & $\begin{array}{c}76 \\
114\end{array}$ & & & $\begin{array}{l}35 \\
55\end{array}$ & \\
\hline & $\mathrm{J}(48) \mathrm{C} 1200$ & 4000 & 96.40 & 228 & & & 110 & \\
\hline
\end{tabular}

Journal of Engineering Sciences, Assiut University, Faculty of Engineering, Vol. 41, No. 3, May, 2013, E-mail address: jes@aun.edu.eg 
L. K. Idriss et al., Static behaviour of different types of R.C beam-column connections as affected by both value acting lateral horizontal force and grade of used concrete (theoretical study) part two, pp.746 - 814

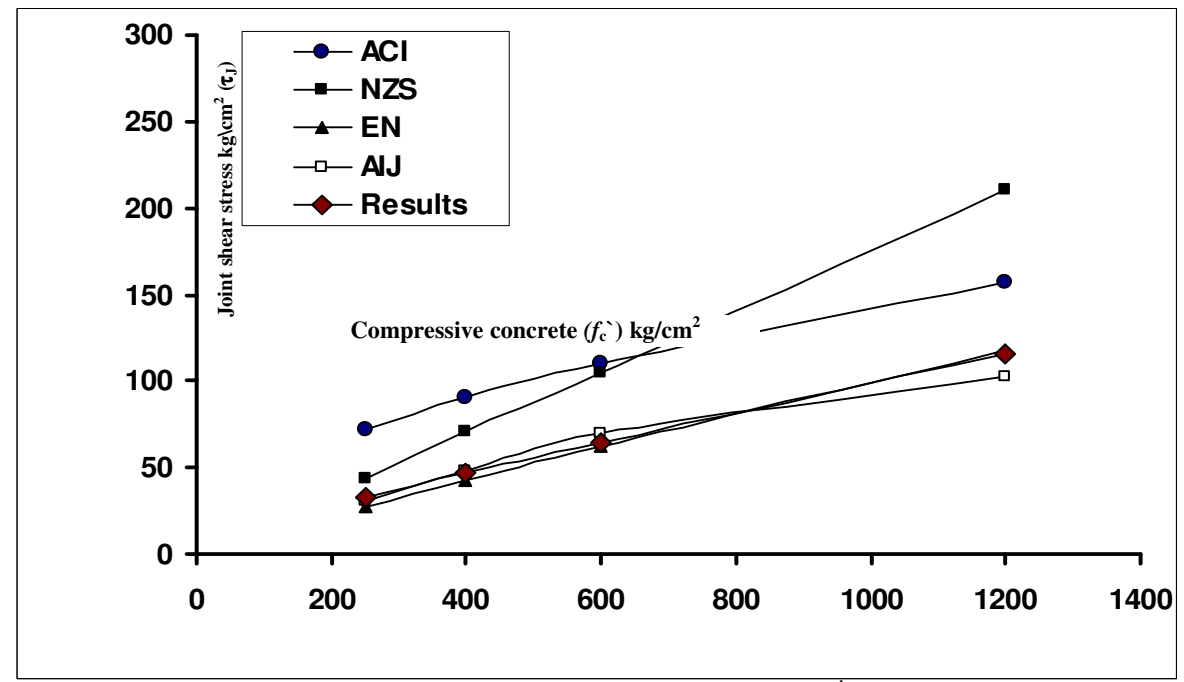

Fig. 23. Relation of Concrete Compressive Strength $\left(f_{\mathrm{c}}\right)$ - Joint Shear Stress $\left(\tau_{\mathrm{j}}\right)$ Exterior Joint

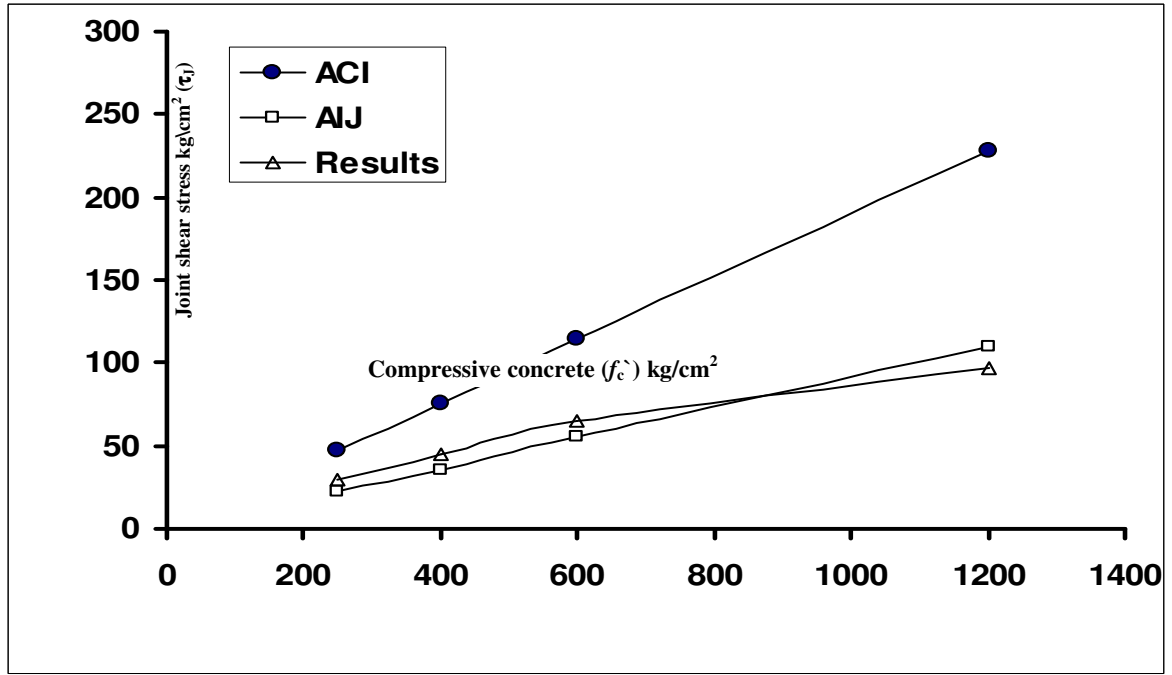

Fig. 24. Relation of Concrete Compressive Strength $\left(f^{\prime} c\right)-$ Joint Shear Stress $(\tau j)$ Knee Joint

AIJ 1997 Architectural Institute of Japan (Design guidelines for earthquake resistant reinforced concrete building based on ultimate strength concept and commentary) has a standard value of the joint shear strength $\left(\tau_{\mathrm{j}}\right)$ is suggested as given by Eq. (10);

$$
\tau_{\mathrm{j}}=0.8 \times\left(\dot{f}_{\mathrm{c}}\right)^{0.7}
$$

Based on same trend of reference it is illustrated that the following equations 11, 12 and 13 can evaluated for our studied joints.

Journal of Engineering Sciences, Assiut University, Faculty of Engineering, Vol. 41, No. 3, May, 2013,E-mail address: jes@aun.edu.eg 
L. K. Idriss et al., Static behaviour of different types of R.C beam-column connections as affected by both value acting lateral horizontal force and grade of used concrete (theoretical study) part two, pp.746 - 814 .

\begin{tabular}{|c|c|}
\hline $\begin{array}{l}\tau_{j u}=1.56 \times \dot{f}_{\mathrm{c}}^{0.673} \\
\tau_{j u}=1.13 \times \dot{f_{\mathrm{c}}} 0.573 \\
\tau_{j u}=1.09 \times \dot{f}_{\mathrm{c}} 0.573\end{array}$ & $\begin{array}{l}\text { Interior beam-column joint } \\
\text { Exterior beam-column joint } \\
\text { Knee beam-column joint }\end{array}$ \\
\hline
\end{tabular}

On the light of Table 7 and Figures 22, 23 and 24 for comparing codes recommendations with our results, it is obtained that:

1. For Interior Joint the maximum shear stress capacity for the studied connections is 69\% lower as per ACI 318M-02 and is lower as 52\% per ECCS 203 (2001) and higher than that provided by NZS 3101:1995 per 67\% and higher than that provided by AIJ 1997 per 52\% and also the same per EN 1998-1:2003.

2. For Exterior Joint the maximum shear stress capacity for the studied connections is 45\% lower as per ACI 318M-02 and is lower as 74\% per NZS 3101:1995 and higher than that provided by per 85\% per EN 1998-1:2003 and higher than that provided by AIJ 1997 per $95 \%$.

3. For Knee Joint the maximum shear stress capacity for studied connections is $63 \%$ lower as per ACI 318M-02 and higher than that provided by AIJ 1997 per 77\%.

\subsubsection{Effect of Concrete Compressive Strength $\left(f^{\prime} c\right)$ on Bond Strength $(\mu b)$.}

A maximum bond stress $\left(u_{b}\right)$ of beam reinforcement over the column width was estimated by assuming simultaneous yielding of the beam reinforcement in tension and compression at the two faces of the joint. The bond strength was assumed proportional to the square root of the concrete compressive strength Table 8 . The beam bar bond index (BI) was defined by dividing the average bond stress by the square root of the concrete strength.

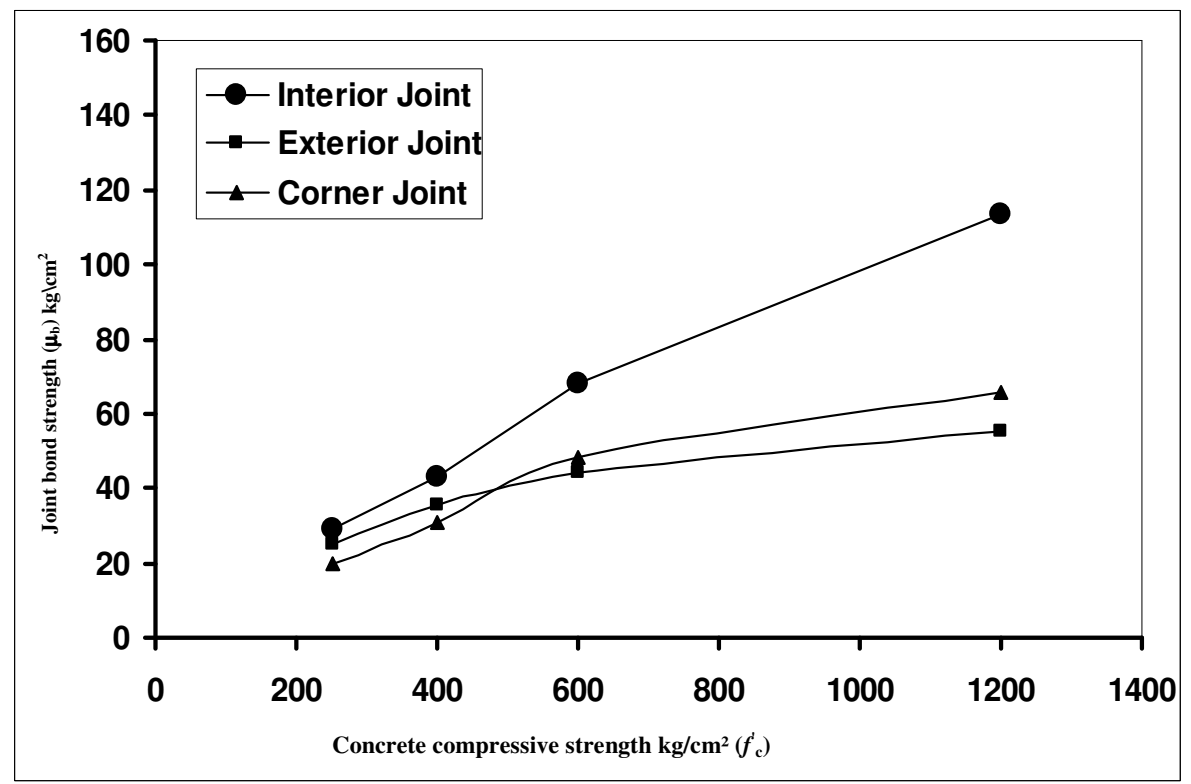

Fig. 25. Relation of Concrete Compressive Strength $\left(\dot{f}_{\mathrm{c}}\right)$-Joint Bond Stress kglcm ${ }^{2}\left(\mu_{\mathrm{b}}\right)$

Journal of Engineering Sciences, Assiut University, Faculty of Engineering, Vol. 41, No. 3, May, 2013,E-mail address: jes@aun.edu.eg 
L. K. Idriss et al., Static behaviour of different types of R.C beam-column connections as affected by both value acting lateral horizontal force and grade of used concrete (theoretical study) part two, pp.746 - 814

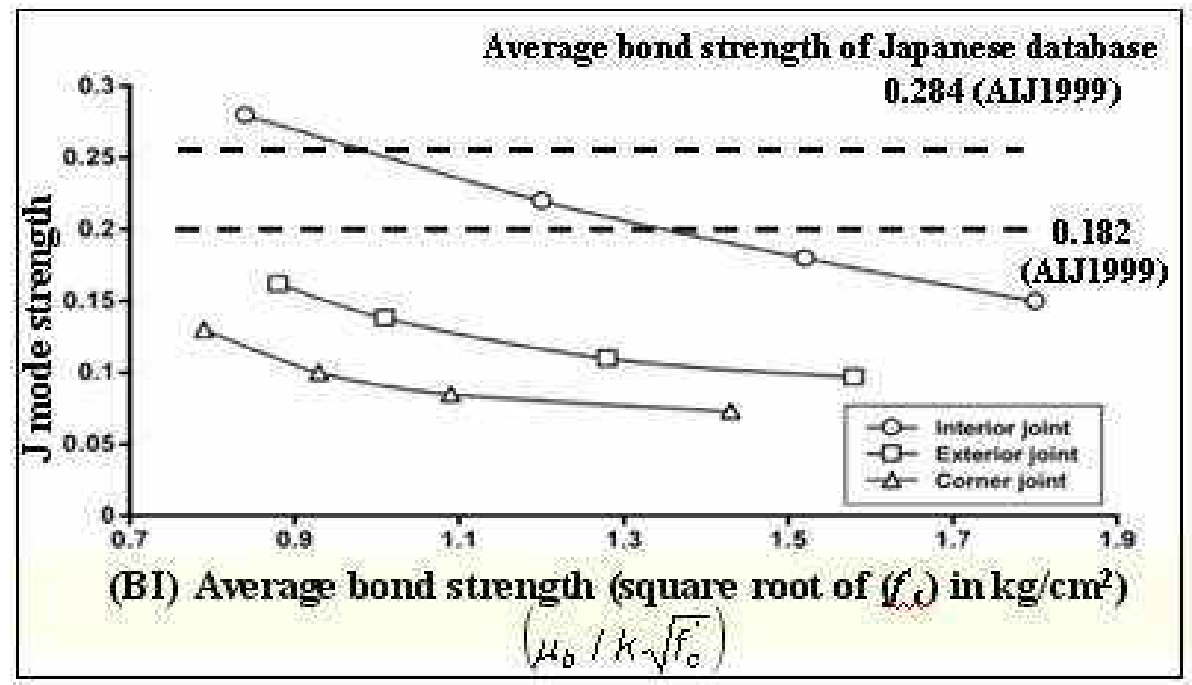

Fig. 26. Relation of A beam bar bond index (BI) - Range of Mode Strength(J) (Effect of Bond Capacity)

\section{Table 8.}

Effect of Bond Capacity of Interior, Exterior and Corner (Knee) Joint at Concrete Compressive Strength.

\begin{tabular}{|c|c|c|c|c|c|c|}
\hline & Joints No. & $\begin{array}{c}\text { Yield strength } \\
\text { of } \\
\text { reinforcement } \\
\left(f_{\mathrm{y}}\right) \mathrm{kg} / \mathrm{cm}^{2}\end{array}$ & $\begin{array}{l}\text { (J) Range } \\
\text { of mode } \\
\text { strength } \\
\left(\tau_{j} \backslash f_{c}^{\prime}\right)=j\end{array}$ & $\begin{array}{l}\text { Average } \\
\text { bond } \\
\text { strength } \\
\left(\mu_{\mathrm{b}}\right) \mathrm{kg} / \mathrm{cm}^{2}\end{array}$ & $\begin{array}{c}\text { (BI) Average bond } \\
\text { strength } / \mathrm{k}=1.8 \\
\text { square root of }\left(f_{\mathrm{c}}^{\prime}\right) \\
\left(\mu_{\mathrm{b}} / \mathrm{k} \sqrt{ } f_{\mathrm{c}}^{\prime}\right)\end{array}$ & $\begin{array}{c}\text { Average bond } \\
\text { strength } \backslash \\
\text { concrete } \\
\text { compressive stress } \\
\left(\mu_{\mathrm{b}} \backslash f_{c}^{\prime}\right)\end{array}$ \\
\hline \multirow{4}{*}{ 总 } & $\mathrm{J}(4) \mathrm{C} 250$ & 2400 & 0.28 & 29.11 & 0.84 & 0.116 \\
\hline & $\mathrm{J}(8) \mathrm{C} 400$ & 2800 & 0.22 & 43.02 & 1.20 & 0.107 \\
\hline & $\mathrm{J}(12) \mathrm{C} 600$ & 3600 & 0.18 & 67.87 & 1.52 & 0.113 \\
\hline & $\mathrm{J}(16) \mathrm{C} 1200$ & 4000 & 0.15 & 113.20 & 1.80 & 0.094 \\
\hline \multirow{4}{*}{ 离 } & $\mathrm{J}(20) \mathrm{C} 250$ & 2400 & 0.162 & 25.20 & 0.88 & 0.11 \\
\hline & $\mathrm{J}(24) \mathrm{C} 400$ & 2800 & 0.138 & 35.66 & 1.01 & 0.089 \\
\hline & J (28)C600 & 3600 & 0.11 & 44.25 & 1.28 & 0.074 \\
\hline & $\mathrm{J}(32 \mathrm{C} 1200$ & 4000 & 0.097 & 55.54 & 1.58 & 0.046 \\
\hline \multirow{4}{*}{ 预. } & $\mathrm{J}(36) \mathrm{C} 250$ & 2400 & 0.13 & 19.69 & 0.79 & 0.078 \\
\hline & $\mathrm{J}(40) \mathrm{C} 400$ & 2800 & 0.10 & 30.59 & 0.93 & 0.076 \\
\hline & J (44)C600 & 3600 & 0.085 & 48.33 & 1.09 & 0.080 \\
\hline & $\mathrm{J}(48) \mathrm{C} 1200$ & 4000 & 0.073 & 65.93 & 1.43 & 0.055 \\
\hline
\end{tabular}

Figure 25 shows the relation between compressive strength of concrete $\left(f_{c}\right)$ and the joint bond stress $\left(\mu_{\mathrm{b}}\right)$ for studied joints for the studied case of loading. The increase of concrete

Journal of Engineering Sciences, Assiut University, Faculty of Engineering, Vol. 41, No. 3, May, 2013, E-mail address: jes@aun.edu.eg 
L. K. Idriss et al., Static behaviour of different types of R.C beam-column connections as affected by both value acting lateral horizontal force and grade of used concrete (theoretical study) part two, pp.746 - 814 .

compressive strength with yielding of the beam reinforcement effective to increase the bond strength.

This result is important, it was observed that the interior joint has maximum bond strength at high concrete compressive strength, it can be denoted that interior joint is stronger than exterior joint.

Figure 26 shows the calculated value of J-mode strength defined by the parameter of $(\mathrm{K})$ .Larger value of $\mathrm{k}$ generally gives larger joint shear strength. The Fig. 26 also compare the calculation and the average strength reported in the commentary of AIJ Guidelines (AIJ 1999) derived from Japanese database of test of beam-column joint beam bar bond index (BI) was introduced to indicate the severity of bond stress relative to the bond strength.

Based on the work of (Kazuhiro K.1991)

$$
\text { Bond index }=(\mathrm{BI})=\frac{\mathrm{u}_{b}}{\sqrt{\mathrm{f}_{c}^{\prime}}}=\frac{f y}{2 \sqrt{f_{c} \backslash}} \frac{d b}{h c}
$$

Based on our results (Table 8, Fig. 25and 26 ) the bond index can be represented by the equations from 15 to 20 for the studied joints as follows:

1. For interior joint: The beam bar bond index (BI) was defined by dividing the average bond stress $\left(\mu_{\mathrm{b}}\right)$ by the square root of the concrete and given by :

$$
\begin{aligned}
& (\mathrm{BI})=\frac{\mathrm{u}_{b}}{\sqrt{\mathrm{f}_{c}^{\prime}}}=\frac{\mathrm{f}_{\mathrm{y}}}{3.5 \sqrt{\mathrm{f}_{c}^{\prime}}} \frac{\mathrm{d}_{b}}{\mathrm{~h}_{c}} \quad \text { for } 2400 \leq f_{\mathrm{y}}<3600 \quad\left(\mathrm{~kg} / \mathrm{cm}^{2}\right) \\
& (\mathrm{BI})=\frac{\mathrm{u}_{b}}{\sqrt{\mathrm{f}_{c}^{\prime}}}=\frac{\mathrm{f}_{\mathrm{y}}}{2 \sqrt{\mathrm{f}_{c}^{\prime}}} \frac{\mathrm{d}_{\mathrm{b}}}{\mathrm{h}_{c}} \quad \text { for } 3600<f_{\mathrm{y}} \leq 4000 \quad\left(\mathrm{~kg} / \mathrm{cm}^{2}\right)
\end{aligned}
$$

where $f_{y}$ : yield strength of beam bars in $\mathrm{kgf} / \mathrm{cm}^{2}, d_{b}$ : diameter of beam bars, $h_{c}$ : column width and $f_{c}^{\prime}$ : concrete compressive strength in $\mathrm{kgf} / \mathrm{cm}^{2}$.

2. For Exterior Joint the bond index (BI) is given by Eqs. (17) and (18):

$$
\begin{aligned}
& (\mathrm{BI})=\frac{u_{\mathrm{b}}}{\sqrt{f_{\mathrm{c}}^{\prime}}}=\frac{f_{y}}{4.50 \sqrt{f_{\mathrm{c}}^{\prime}}} \frac{d_{b}}{h_{\mathrm{c}}} \quad \text { for } 2400 \leq f_{\mathrm{y}}<3600 \quad\left(\mathrm{~kg} / \mathrm{cm}^{2}\right) \\
& (\mathrm{BI})=\frac{u_{\mathrm{b}}}{\sqrt{f_{\mathrm{c}}^{\prime}}}=\frac{f_{y}}{3.20 \sqrt{f_{\mathrm{c}}^{\prime}}} \frac{d_{b}}{h_{\mathrm{c}}} \quad \text { for } 3600<f_{\mathrm{y}} \leq 4000 \quad\left(\mathrm{~kg} / \mathrm{cm}^{2}\right)
\end{aligned}
$$

3. For Knee Joint the bond index (BI) is given by Eqs. (19) and (20):

$$
(\mathrm{BI})=\frac{u_{\mathrm{b}}}{\sqrt{f_{\mathrm{c}}^{\prime}}}=\frac{f_{y}}{6.20 \sqrt{f_{\mathrm{c}}^{\prime}}} \frac{d_{b}}{h_{c}} \text { for } 2400 \leq f_{y}<3600\left(\mathrm{~kg} / \mathrm{cm}^{2}\right)
$$

Journal of Engineering Sciences, Assiut University, Faculty of Engineering, Vol. 41, No. 3, May, 2013,E-mail address: jes@aun.edu.eg 
L. K. Idriss et al., Static behaviour of different types of R.C beam-column connections as affected by both value acting lateral horizontal force and grade of used concrete (theoretical study) part two, pp.746 - 814

$$
(\mathrm{BI})=\frac{u_{\mathrm{b}}}{\sqrt{f_{\mathrm{c}}^{\prime}}}=\frac{f_{y}}{3.20 \sqrt{f_{\mathrm{c}}^{\prime}}} \frac{d_{b}}{h_{c}} \quad \text { for } 3600<f_{\mathrm{y}} \leq 4000\left(\mathrm{~kg} / \mathrm{cm}^{2}\right)
$$

By comparing our results by AIJ Guidelines.

$$
\mathrm{u}_{\mathrm{b}}=\mathrm{k} \sqrt{\mathrm{f}_{c}^{\prime}} \sum \phi \mathrm{D}_{\mathrm{b}}
$$

Where $: \Sigma \varphi$ is the total perimeter length of longitudinal bar in the first layer of beam side in the column in $\mathrm{mm}$, and $D_{b}$ : beam depth in $\mathrm{mm}$, The value of $(k)$ of 1.8 for modeling of bond capacity of non yielding tensile bar passing beam-column joint by (Lowes, L. 2002).

Finally the value of $(k)$ was suggested for chosen modeling of bond capacity using the following equation for different types of joints;

$$
u_{b}=k \sqrt{f_{\mathrm{c}}^{\prime}}
$$

where $(k)$ is a constant depends on used grade of concrete as given in the following Table.

Table 9.

Suggested Values of $(k)$ for Calculating Average Bond Capacity of R.C. Joints.

\begin{tabular}{lcc}
\hline \hline \multirow{1}{*}{ Type of Joint } & $(\boldsymbol{k})$ values of $\left(\boldsymbol{f}_{\boldsymbol{c}}^{\prime}\right)$ & \\
& $\mathbf{C 2 5 0}$ and $\mathbf{C 4 0 0}$ & $\mathbf{C 6 0 0}$ and $\mathbf{C 1 2 0 0}$ \\
\hline Interior joint & 1.80 & 3.20 \\
Exterior joint & 1.58 & 1.70 \\
Knee joint & 1.20 & 1.92 \\
\hline \hline
\end{tabular}

From the above results and values, it is obvious that for any used grade of concrete interior beam-column joint has larger safety margin than exterior beam-column joint. The same result was found in reference (Hitoshi Sh. 2004).

Figure 27 illustrates the relation between the concrete compressive stress $\left(\dot{f}_{\mathrm{c}}\right)$ and the corresponding induced ratio of compressive stress /bond stress $\left(\left(\mu_{\mathrm{b}} \dot{f}_{\mathrm{c}}\right)\right.$. Figure 27 indicates that the increase of concrete grade is usually accompanied by decreased in the induced $\left(\mu_{\mathrm{b}} \backslash \dot{f}_{\mathrm{c}}\right)$. Also its obvious that the interior joints posses the higher value of $\left(\left(\mu_{\mathrm{b}} \backslash \dot{f}_{\mathrm{c}}\right.\right.$ than that for exterior and corner joint corresponding to the increase of concrete grade $\left(f_{\mathrm{c}}{ }^{\prime}\right)$.

Journal of Engineering Sciences, Assiut University, Faculty of Engineering, Vol. 41, No. 3, May, 2013,E-mail address: jes@aun.edu.eg 
L. K. Idriss et al., Static behaviour of different types of R.C beam-column connections as affected by both value acting lateral horizontal force and grade of used concrete (theoretical study) part two, pp.746 - 814.

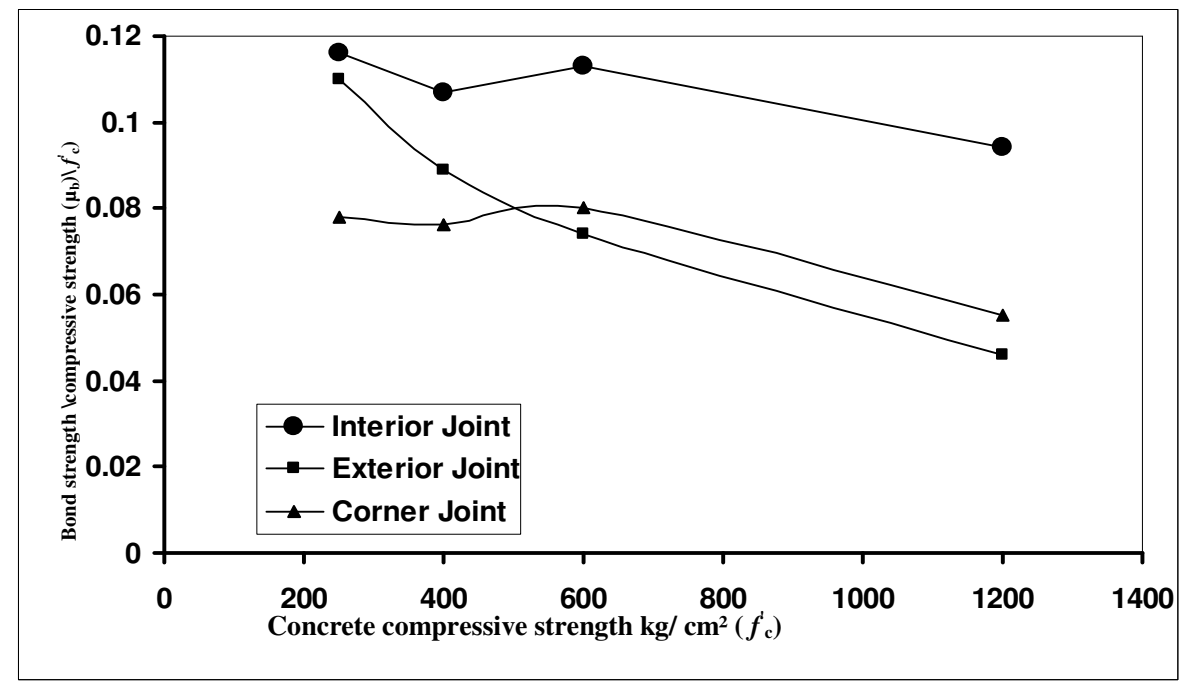

Fig. 27. Relation of Concrete Compressive Strength $\left(\dot{f}_{\mathrm{c}}\right)-$ Bond Stress Compressive Strength $\left(\mu_{\mathrm{b}} \backslash \dot{f}_{\mathrm{c}}\right)$ for Studied Joints

\subsubsection{Effect of Concrete Compressive Strength $\left(\dot{f}_{c}\right)$ on Maximum Principle Stresses $\left(\sigma_{l}\right)$ :}

Figure 28 and Table 5 show maximum principle stresses values of the joint concrete at the maximum strength for peak loading for interior, exterior and knee joint. The increase of concrete compressive strength is effective to increase the principle stresses.

For $\mathrm{C} 250$ and $\mathrm{C} 400$, the principle stresses for exterior and knee joint is $62 \%$ that for interior beam column joint, however for $\mathrm{C} 600$ the principle stress for exterior and knee joint equals 57\% that for interior beam column joint. Finally for C1200 the principle stress for exterior and knee joint is about $43 \%$ of that for interior beam column joint.

(Hitoshi Sh. 2005) stated that the strength of the exterior beam-column joints is 53\% of the strength of the interior beam-column joint .

Journal of Engineering Sciences, Assiut University, Faculty of Engineering, Vol. 41, No. 3, May, 2013, E-mail address: jes@aun.edu.eg 
L. K. Idriss et al., Static behaviour of different types of R.C beam-column connections as affected by both value acting lateral horizontal force and grade of used concrete (theoretical study) part two, pp.746 - 814

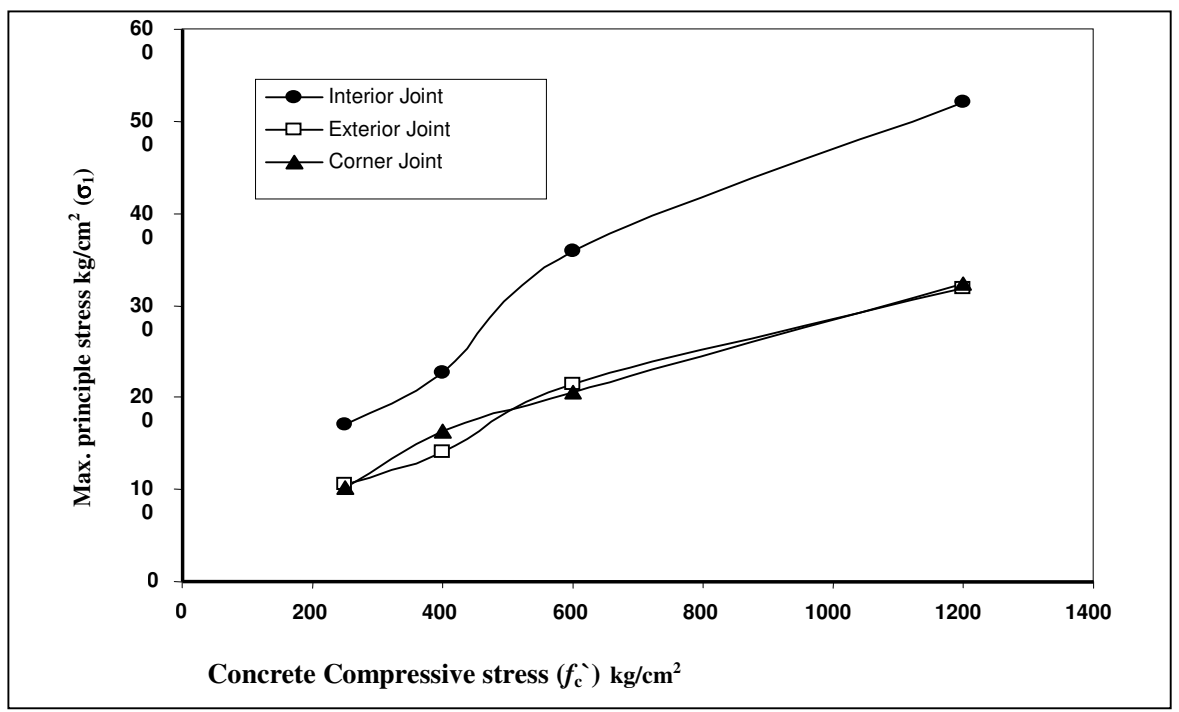

Fig.28. Relation Concrete Compressive Strength $\left(\dot{f}_{c}\right)$ - Maximum Principle $\operatorname{Stress}\left(\sigma_{1}\right)$

Figure 29 illustrates that as the ratio of $\left(\sigma_{1} \backslash f^{\prime}{ }_{c}\right)$ decreases with the corresponding concrete compressive strength $\left(f^{\prime}{ }_{c}\right)$ increases for $(\mathrm{C} 250-\mathrm{C} 400)$ for interior ,exterior and corner joint, however the ratio of $\left(\sigma_{1} \backslash f_{c}^{\prime}\right)$ increases with the corresponding concrete compressive strength $\left(f_{\mathrm{c}}^{\prime}\right)$ increases from $(\mathrm{C} 400-\mathrm{C} 600)$. Also it is obvious that the interior joints posses higher joint ratio of $\left(\sigma_{1} \backslash f^{\prime}\right)$ in comparison with that for both corner and exterior joint respectively.

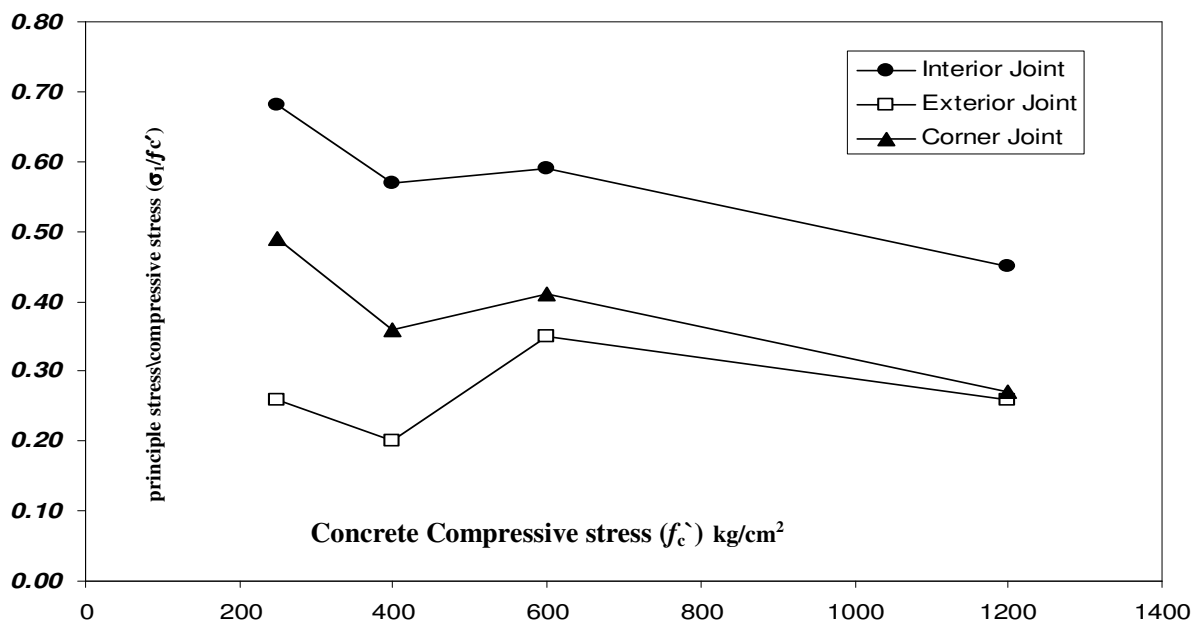

Fig.29. Relation of Concrete Compressive Strength $\left(f_{\mathrm{c}}^{\prime}\right)$ - Maximum Principle Stress $\backslash$ Concrete Compressive Strength $\left(\sigma_{1} \backslash f_{\mathrm{c}}\right)$ for Studied Joints

Journal of Engineering Sciences, Assiut University, Faculty of Engineering, Vol. 41, No. 3, May, 2013,E-mail address: jes@aun.edu.eg 
L. K. Idriss et al., Static behaviour of different types of R.C beam-column connections as affected by both value acting lateral horizontal force and grade of used concrete (theoretical study) part two, pp.746 - 814 .

\subsection{W.R.T Deformations and Strains Points of View:}

\subsection{Effect of Concrete Compressive Strength $\left(\dot{f}_{c}\right)$ on Joint Deformation (horizontal} displacement ( $\delta$ ), Drift angle ( $\theta \%)$ Ratio and Shear Strain ( $\% \%)$ \{Distortion\}:

1. It can be seen according to the Table 10 and Figure30, that: as the compressive strength increases the horizontal displacement decreases for studied joints. Concrete compressive strength has a moderate effect to decrease horizontal displacement.

2. Based on Figure 31 it is obvious that the increase of concrete grade $\left(f_{\mathrm{c}}{ }^{\prime}\right)$ is usually accompanied with a decrease of the induced drift angle $(\theta \%)$ for studied type of R.C joints. Also the induced drift angle $(\theta \%)$, for a given grade of concrete $\left(f_{\mathrm{c}}{ }^{\prime}\right)$, mainly depends on the type of joint. The interior joint possesses the smallest value of drift angle $(\theta \%)$ in comparison with that for exterior or corner joint. The corner joint possess the higher value of drift angle $(\theta \%)$.

3. It is obvious that on light of Figure 32 the increase of concrete grade $\left(f_{\mathrm{c}}{ }^{\prime}\right)$ is usually accompanied with a slight decrease of joint shear distortion $(\gamma \%)$. At the same time the corner joint possess higher value compared with that for both exterior and interior joints. The minimum value of joint shear distortion $(\gamma \%)$ is corresponding to the interior joints

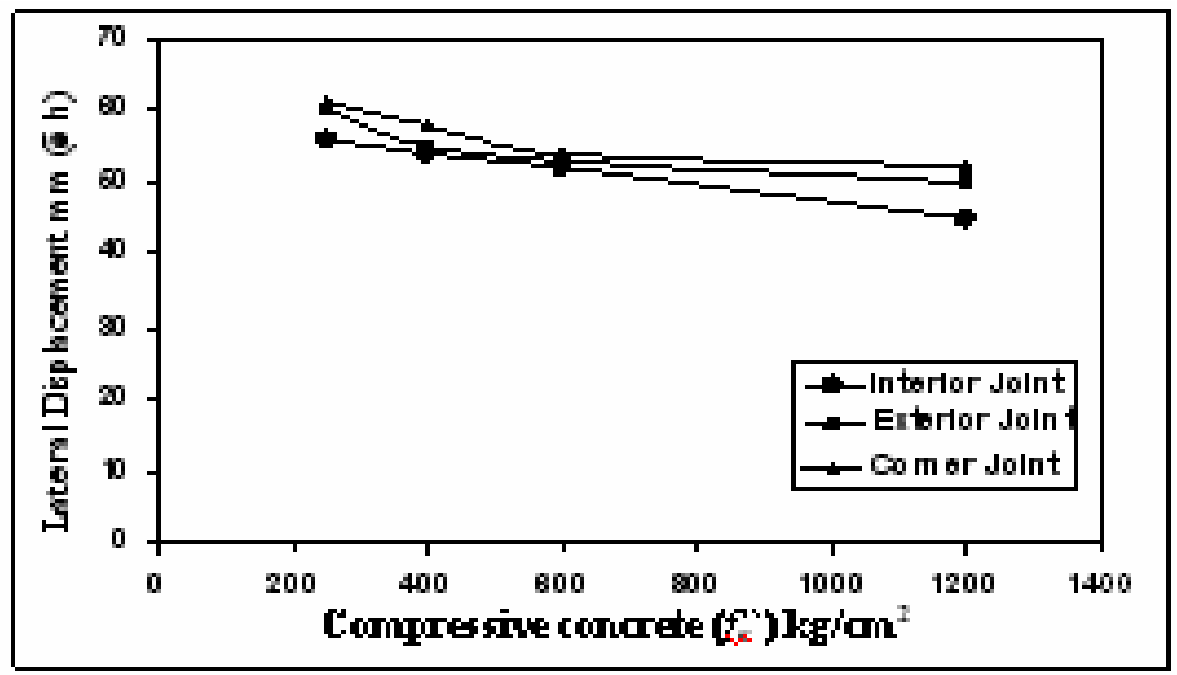

Fig. 30. Relation of Concrete Compressive Strength $\left(f^{\prime}{ }^{\prime}\right)$-Horizontal Displacement (ठh)mm

Journal of Engineering Sciences, Assiut University, Faculty of Engineering, Vol. 41, No. 3, May, 2013,E-mail address: jes@aun.edu.eg 
L. K. Idriss et al., Static behaviour of different types of R.C beam-column connections as affected by both value acting lateral horizontal force and grade of used concrete (theoretical study) part two, pp.746 - 814

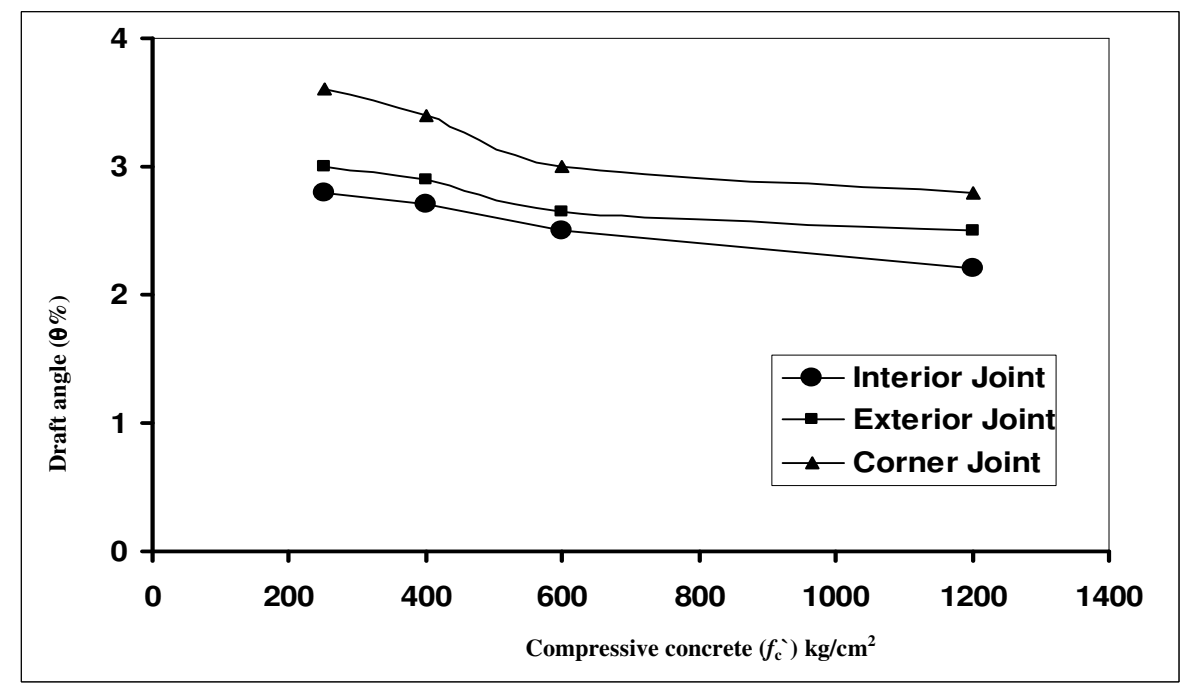

Fig. 31. Relation of Concrete Compressive Strength $\left(f^{\prime}{ }_{c}\right)$-Drift angle $(\theta \%)$

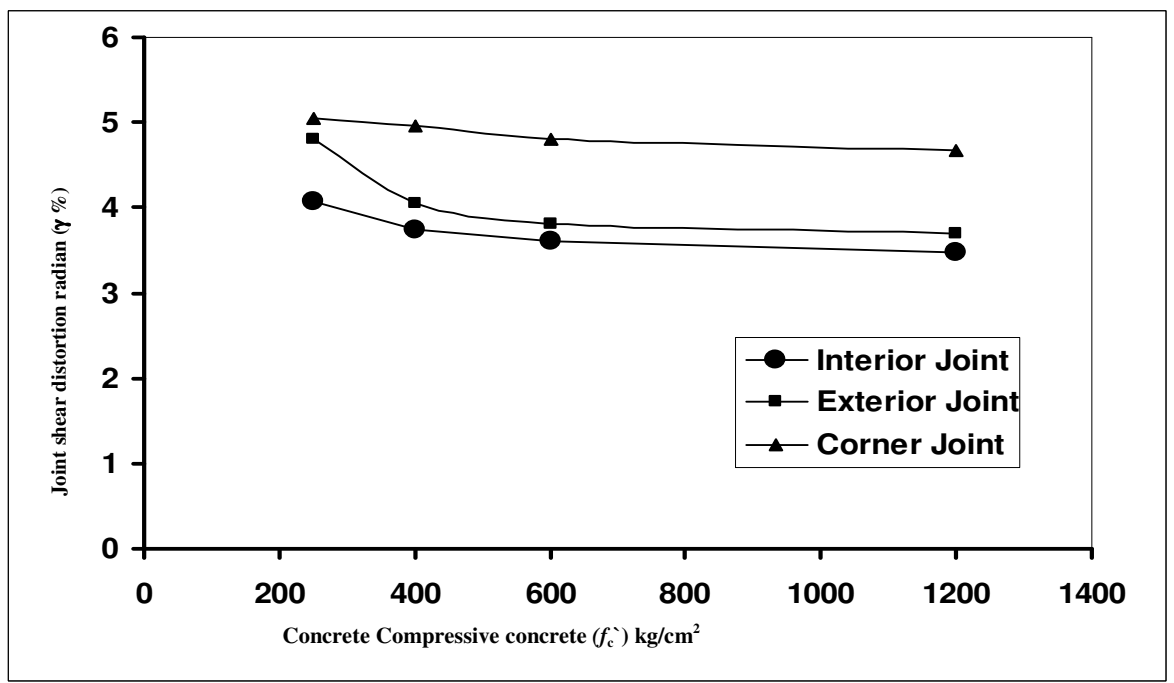

Fig. 32. Relation of Concrete Compressive Strength $\left(f^{\prime}{ }_{c}\right)-$ Joint Shear Distortion radian $(\gamma \%)$

\subsection{Effect of Concrete Compressive Strength ( $\left.f c^{\prime}\right)$ on the Maximum Principal Strains ( $(\varepsilon 1)$ :}

Figure 33 and Table 10 indicates that the increase of concrete grade is usually accompanied by an increase in the induced principle strains disregarding the type of joint. Also it is interesting to note that Interior joint usually possess higher values of maximum induced principle strain for any grade of concrete. The smallest values of maximum

Journal of Engineering Sciences, Assiut University, Faculty of Engineering, Vol. 41, No. 3, May, 2013,E-mail address: jes@aun.edu.eg 
794

L. K. Idriss et al., Static behaviour of different types of R.C beam-column connections as affected by both value acting lateral horizontal force and grade of used concrete (theoretical study) part two, pp.746 - 814 .

principle strains are corresponding to corner joints. Also Figure 33indicates that the rate of increase of maximum principle strain with respect to the increase of concrete grade is more or less equal for all kinds of studied joints.

Figure 34 indicates that the increase of concrete grade is usually accompanied by an increase in the induced principle stress / principle strain ratio $\left(\sigma_{1} / \varepsilon_{1}\right)$, However, concrete compressive strength $\left(f^{\prime}\right)$ has a slight effect to increase $\left(\sigma_{1} / \varepsilon_{1}\right)$ for corner joints. Also it is obvious that an interior joints and exterior joint posses the same ratio of increasing the $\left(\sigma_{1} / \varepsilon_{1}\right)$ corresponding to the increasing of concrete grade $\left(f_{\mathrm{c}}{ }^{\prime}\right)$.

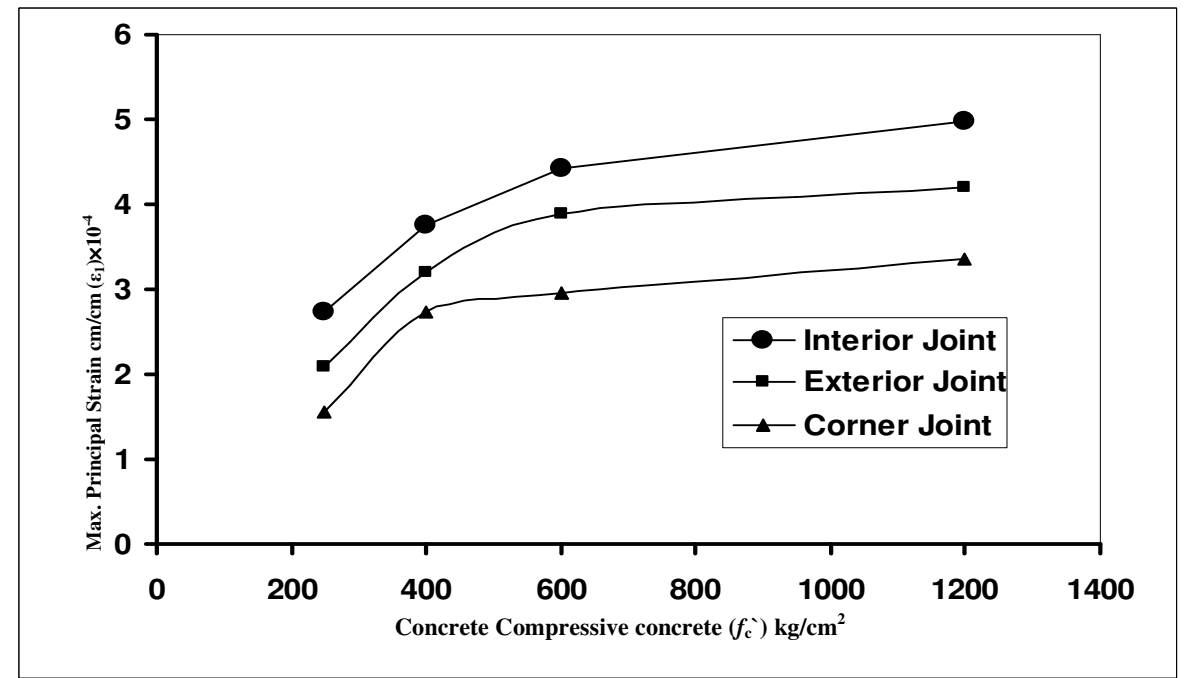

Fig. 33. Relation of Concrete Compressive Strength $\left(f^{\prime}{ }_{c}\right)$ - Max. Principal Strain $\left(\varepsilon_{1}\right) \mathrm{cm} / \mathrm{cm}$

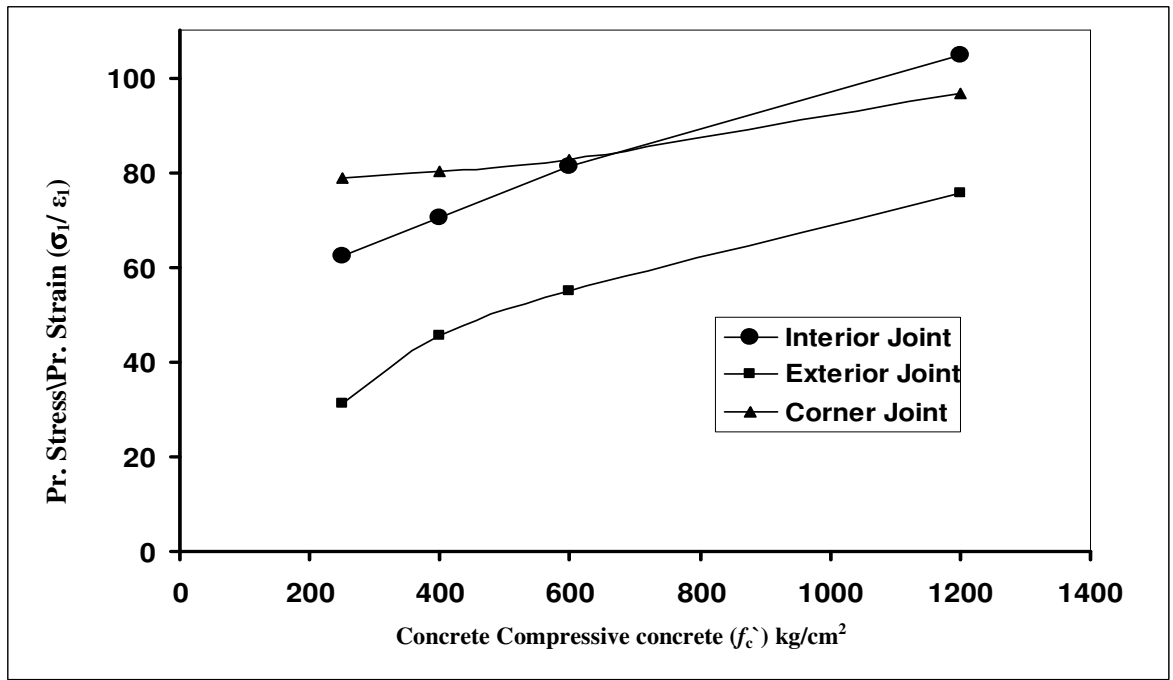

Fig. 34. Relation of Concrete Compressive Stress $\left(f_{\mathrm{c}}{ }^{\prime}\right)$ - Ratio of Maximum Principle Stress / Maximum Principle Strain $\left(\sigma_{1} / \varepsilon_{1}\right)$ for Studied Joints

Journal of Engineering Sciences, Assiut University, Faculty of Engineering, Vol. 41, No. 3, May, 2013,E-mail address: jes@aun.edu.eg 
L. K. Idriss et al., Static behaviour of different types of R.C beam-column connections as affected by both value acting lateral horizontal force and grade of used concrete (theoretical study) part two, pp.746 - 814

Table 10.

Effect of Concrete Compressive Strength on Deformations and Strains of Beam-Column Joint.

\begin{tabular}{|c|c|c|c|c|c|c|c|c|c|c|c|}
\hline & Joint No.t & $\begin{array}{c}\text { Yield } \\
\text { strength } \\
\left(f_{y}\right) \\
\mathrm{kg} / \mathrm{cm}^{2}\end{array}$ & $\begin{array}{l}\mathbf{N} / \\
f_{\mathrm{e}} \mathbf{A}_{\mathrm{c}}\end{array}$ & $\begin{array}{c}\text { Lateral } \\
\text { loadton } \\
\left(V_{c}\right)\end{array}$ & $\begin{array}{l}\text { Draft } \\
\text { angle } \\
(\theta \%)\end{array}$ & $\begin{array}{c}\text { Top } \\
\text { lateral } \\
\text { Displacem } \\
\text { ent } \\
\text { mm(סh) }\end{array}$ & $\begin{array}{c}\text { Max } \\
\text { principle } \\
\text { strain } \\
\text { cm/cm } \\
\left(\varepsilon \times 10^{-4}\right)\end{array}$ & $\begin{array}{c}\text { Jointshear } \\
\text { distortion } \\
\text { radian } \\
(\gamma \%)\end{array}$ & 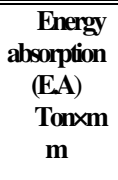 & $\begin{array}{l}\text { Max. } \\
\text { principle } \\
\text { stress } \\
\left(\text { kglcm }^{2}\right. \\
)\left(\sigma_{1}\right)\end{array}$ & $\begin{array}{c}\text { Max } \\
\text { PristresslMax } \\
\text { Pr.Strain } \\
\left(\sigma \mid \sigma_{1}\right) \times \\
10 \mathrm{~kg} / \mathrm{cm}^{2}\end{array}$ \\
\hline \multirow{2}{*}{ 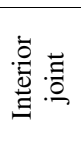 } & $\mathrm{J}(4) \mathrm{C} 250$ & 2400 & 0.15 & 50 & 2.80 & 56 & 2.73 & 4.08 & 1200 & 170.74 & 62.54 \\
\hline & $\mathrm{J}(8) \mathrm{C} 400$ & 2800 & 0.15 & 50 & 2.70 & 54 & 3.76 & 3.75 & 1480 & 263 & 70.13 \\
\hline \multirow{4}{*}{ 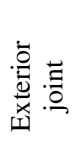 } & $\mathrm{J}(20) \mathrm{C} 250$ & 2400 & 0.15 & 50 & 3.00 & 60 & 2.08 & 4.80 & 890 & 65 & 31.25 \\
\hline & $\mathrm{J}(24) \mathrm{C} 400$ & 2800 & 0.15 & 50 & 2.90 & 55 & 3.19 & 4.05 & 1200 & 145.2 & 45.52 \\
\hline & $\mathrm{J}(28) \mathrm{C} 600$ & 3600 & 0.15 & 50 & 2.65 & 53 & 3.89 & 3.80 & 1580 & 214.6 & 55.16 \\
\hline & $\mathrm{J}(32 \mathrm{C} 1200$ & 4000 & 0.15 & 50 & 2.50 & 50 & 4.20 & 3.70 & 2028 & 318 & 75.71 \\
\hline 离. & $\mathrm{J}(36) \mathrm{C} 250$ & 2400 & 0.15 & 50 & 3.60 & 61 & 1.56 & 5.05 & 595 & 122.95 & 78.82 \\
\hline
\end{tabular}

\subsection{W.R.T Energy absorption (E.A)point of view:}

On the light of Figure 35 and Table 10, it is obvious that as the compressive strength of used concrete increases the dissipated energy considerably increases disregarding the type of joint. Mean while for a given concrete grade the dissipated energy is higher for interior joint rather than that exterior joint, at the same time the later one shows a larger value than that for corner joint.

Meanwhile for C400 and C250 it has more higher per 175\% for interior joint than that for exterior and knee joint. It can also be denoted that interior joint for C1200 is stronger and possesses more ductility of than that for other joints because this joint absorbed the highest total energy amount.

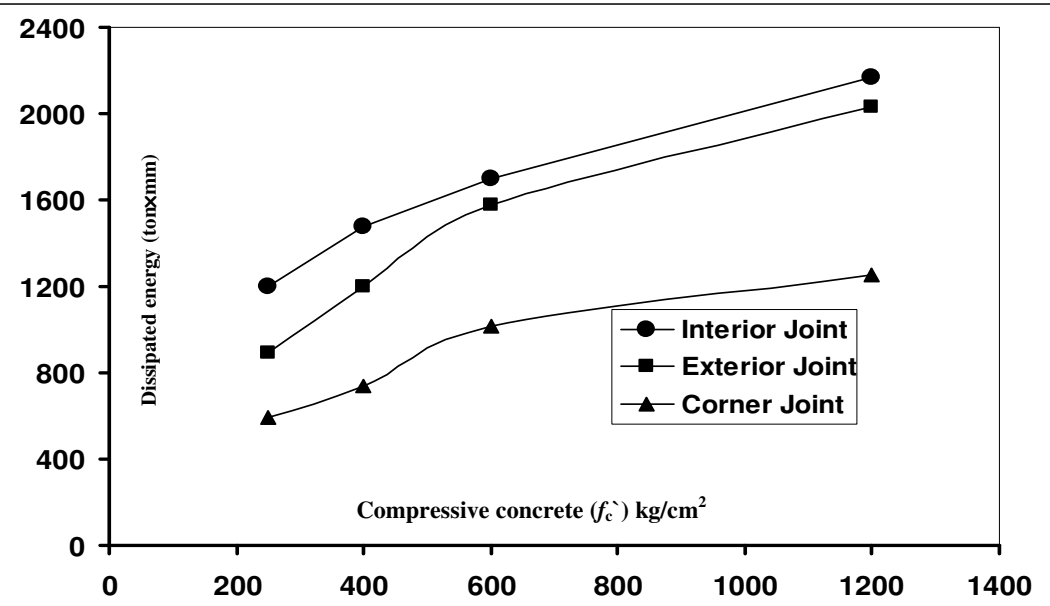

Fig. 35. Relation of Concrete Compressive Strength $\left(f_{c}^{\prime}\right)$-Dissipated energy (E.A) (ton $\left.\times m m\right)$

Journal of Engineering Sciences, Assiut University, Faculty of Engineering, Vol. 41, No. 3, May, 2013,E-mail address: jes@aun.edu.eg 
L. K. Idriss et al., Static behaviour of different types of R.C beam-column connections as affected by both value acting lateral horizontal force and grade of used concrete (theoretical study) part two, pp.746 - 814 .

8.4.W.R.T mode of failure of beam-column joints:

Based on the obtained results it is obvious that for the case of studied loading systems three different modes of failure were observed .Such modes are characterized as follows and shown in Fig. 36,37 and 38:

1. $\quad$ (B)-mode $=$ beam bond failure ,If joint shear increase keeping constant bond resistance, the beam bars slip into the joint at the beam end in compressive side, while bars slip out in tensile side. By considering the deformation compatibility in beam bars and concrete (beam flexure yielding), the elongation of beam bars cause opening of crack in concrete $\mathrm{J}(2,4,6,8)$ for interior joint, $\mathrm{J}(18,20,32)$ Exterior joint and $(34,36)$ for Knee joint.

2. $\quad(\mathbf{J})$-mode $=$ joint shear failure, in the beam-column connection occurs when Joint shear exceeds the shear strength of the beam-column connection. Failure models using the truss mechanism or the strut mechanism regard thus rotational movements of the segments cause uneven opening of the diagonal cracks like flexural cracks $J(10,12,16)$ for interior joint , $\mathrm{J}(24,26,30)$ Exterior joint and $(34,36)$ for Knee joint.

3. (JB)-mode $=$ Plastic hinge failure The next generation of beam-column joint models decoupled the inelastic response of the beams, joint moment-rotation data from beamcolumn anchorage failure for longitudinal reinforcement embedded in the joint controls inelastic joint action under earthquake loading $\mathrm{J}(14)$ for interior joint, $\mathrm{J}(28)$ Exterior joint and $(42,44)$ for Knee joint.

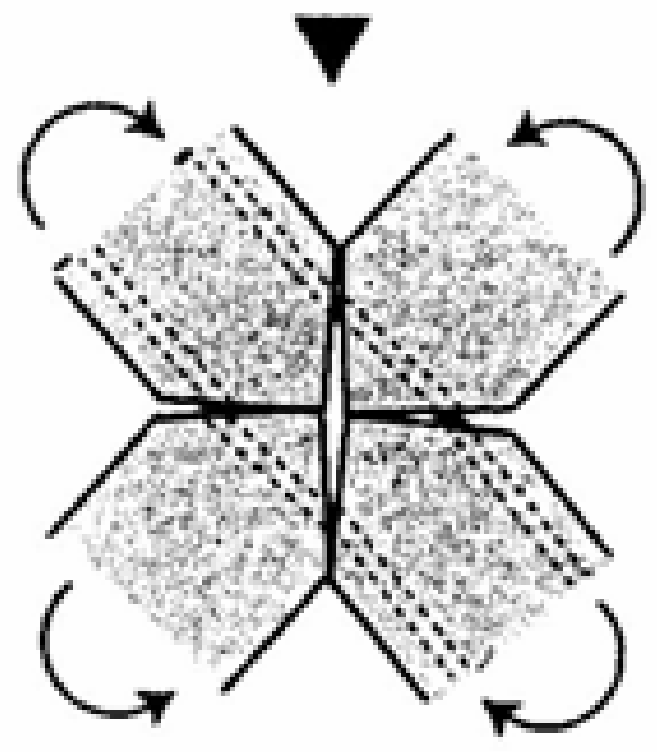

Journal of Engineering Sciences, Assiut University, Faculty of Engineering, Vol. 41, No. 3, May, 2013,E-mail address: jes@aun.edu.eg 
L. K. Idriss et al., Static behaviour of different types of R.C beam-column connections as affected by both value acting lateral horizontal force and grade of used concrete (theoretical study) part two, pp.746 - 814

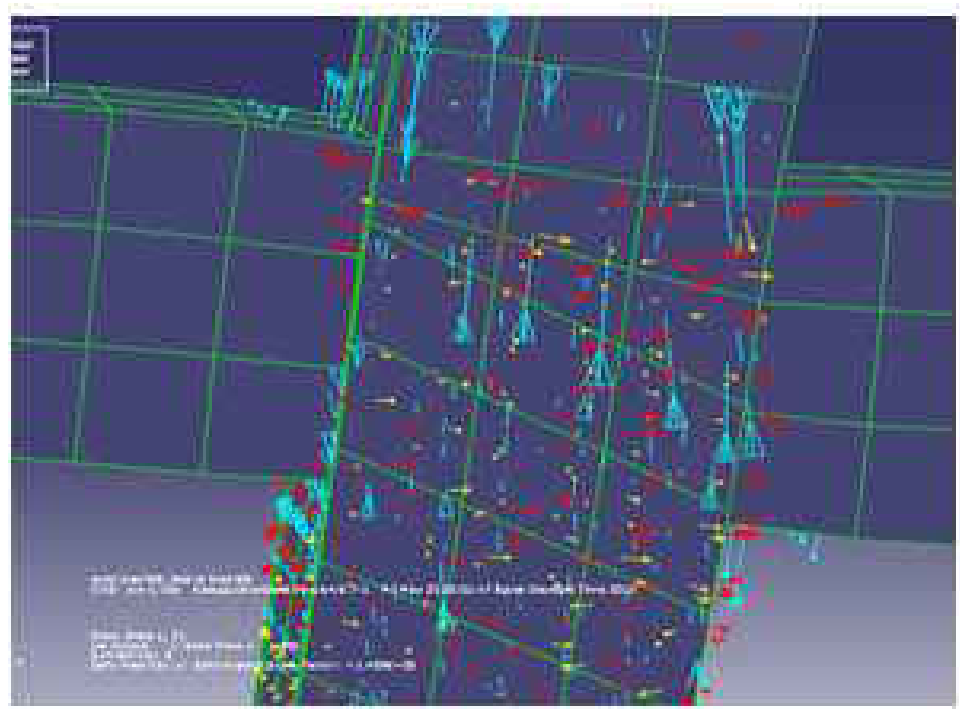

Fig.36. a Deformation Mode by

(B)-mode (Hitoshi Sh. 2005)

(B)-mode Joint No.(2)-obtained results

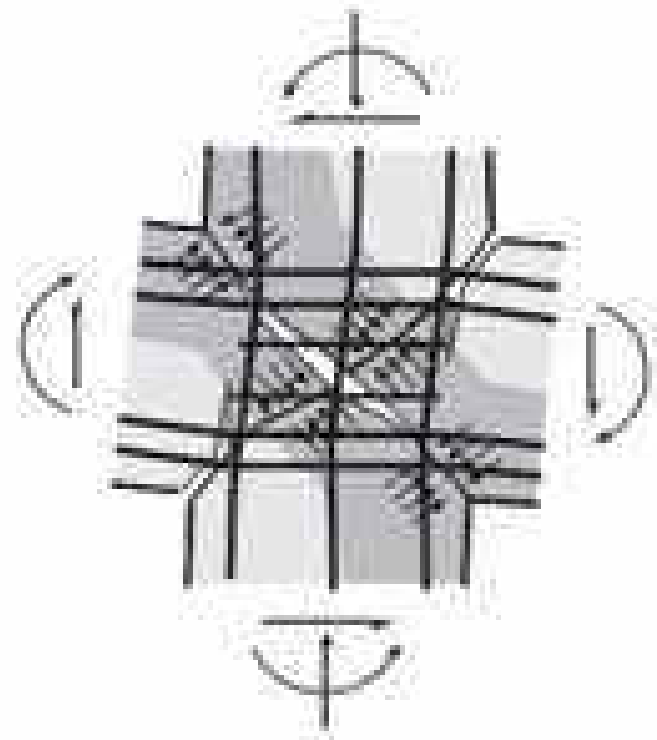

(JB)-mode (Hitoshi Sh. 2005)

Journal of Engineering Sciences, Assiut University, Faculty of Engineering, Vol. 41, No. 3, May, 2013,E-mail address: jes@aun.edu.eg 
L. K. Idriss et al., Static behaviour of different types of R.C beam-column connections as affected by both value acting lateral horizontal force and grade of used concrete (theoretical study) part two, pp.746 - 814.

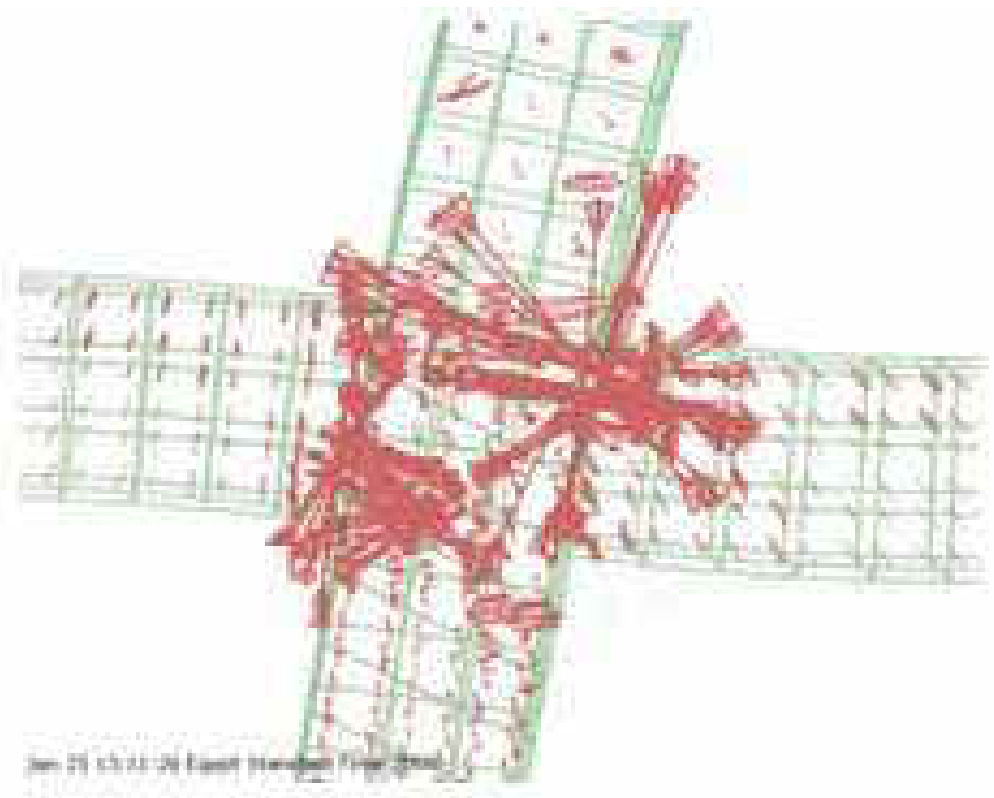

Fig.36.b Deformation Mode by

(JB)-mode (Hitoshi Sh. 2005)

(JB)-mode Joint No.(14) obtained results

\section{Beam end cracks}

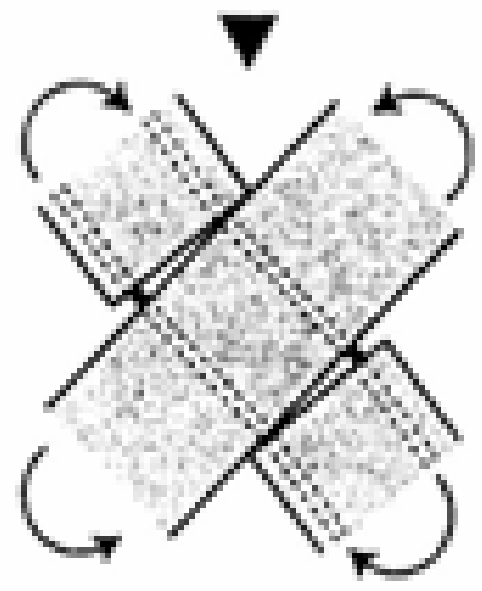

B(Beam) Mode

Journal of Engineering Sciences, Assiut University, Faculty of Engineering, Vol. 41, No. 3, May, 2013,E-mail address: jes@aun.edu.eg 
L. K. Idriss et al., Static behaviour of different types of R.C beam-column connections as affected by both value acting lateral horizontal force and grade of used concrete (theoretical study) part two, pp.746 - 814

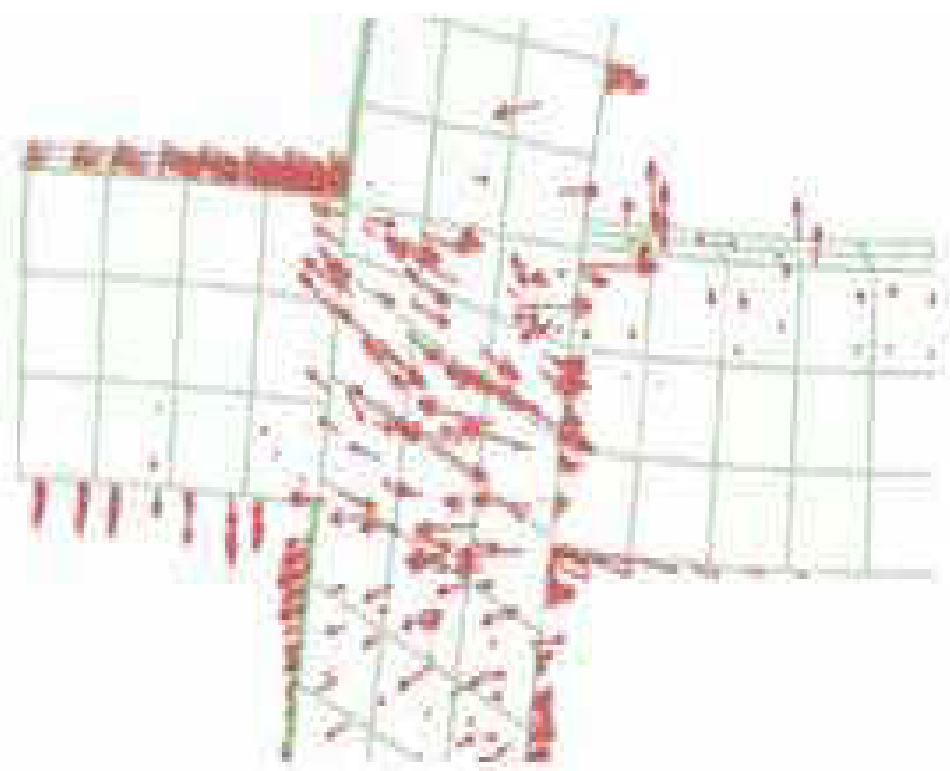

Fig.36.c Deformation Mode by

(J)-mode (Hitoshi Sh. 2004)

(J)-mode Joint No.(16) -obtained results

Fig. 36. Analytical Crack Patterns at Final Loading Stage for Interior BeamColumn Joint

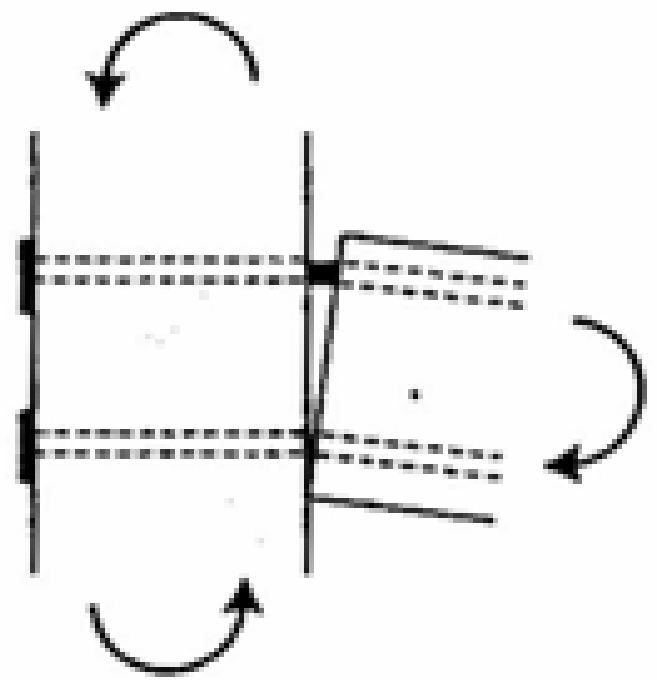

Journal of Engineering Sciences, Assiut University, Faculty of Engineering, Vol. 41, No. 3, May, 2013,E-mail address: jes@aun.edu.eg 
L. K. Idriss et al., Static behaviour of different types of R.C beam-column connections as affected by both value acting lateral horizontal force and grade of used concrete (theoretical study) part two, pp.746 - 814.

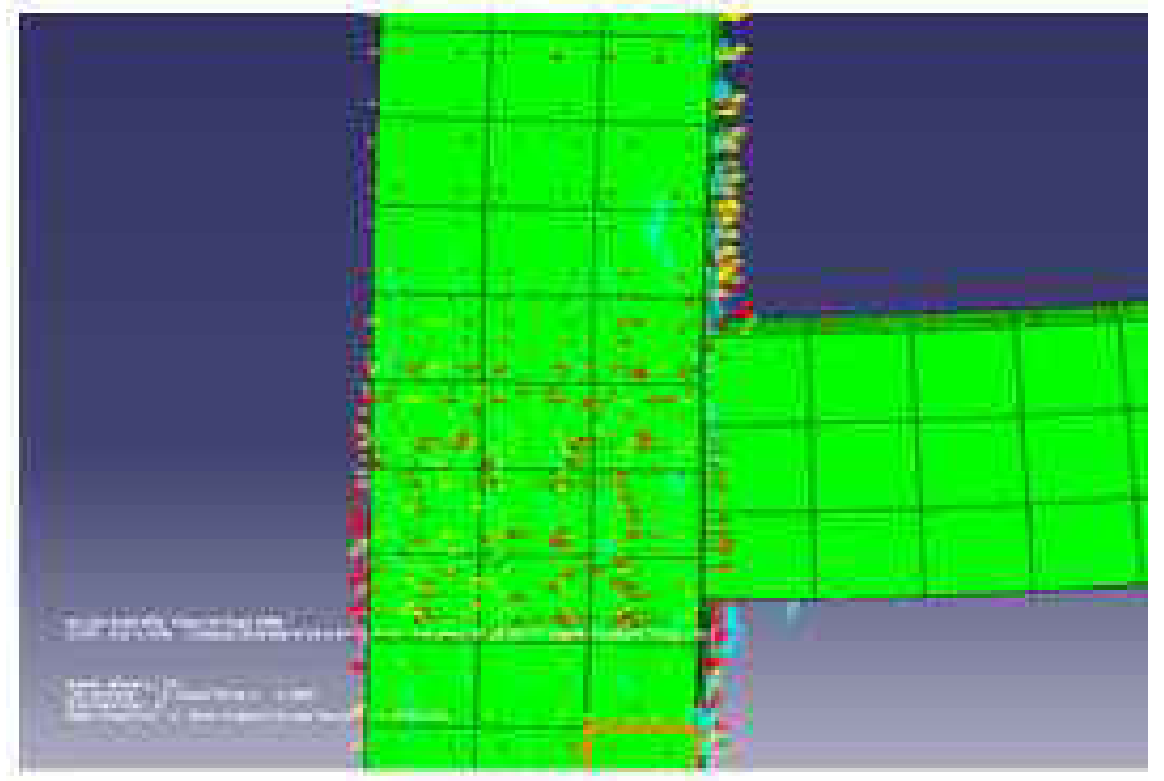

Fig.37.a Deformation Mode by

(B)-mode (Hitoshi Sh. 2005)

(B)-mode Joint No.(21)-obtained results

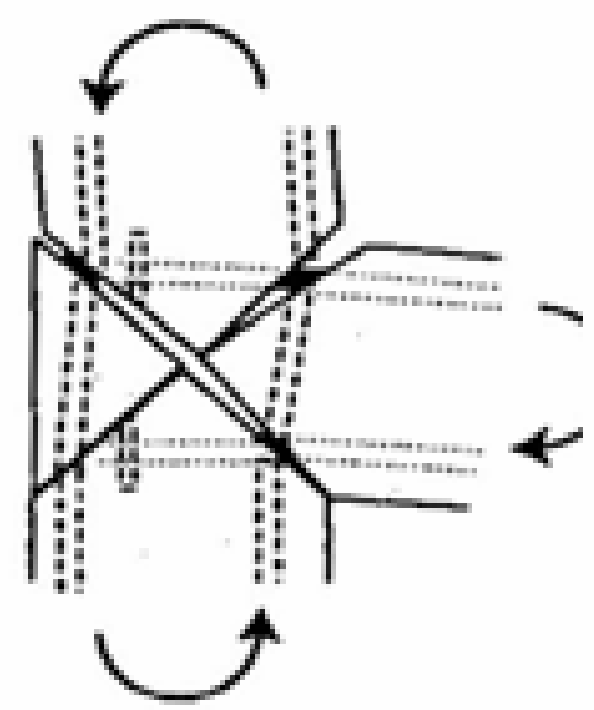

Journal of Engineering Sciences, Assiut University, Faculty of Engineering, Vol. 41, No. 3, May, 2013,E-mail address: jes@aun.edu.eg 
L. K. Idriss et al., Static behaviour of different types of R.C beam-column connections as affected by both value acting lateral horizontal force and grade of used concrete (theoretical study) part two, pp.746 - 814

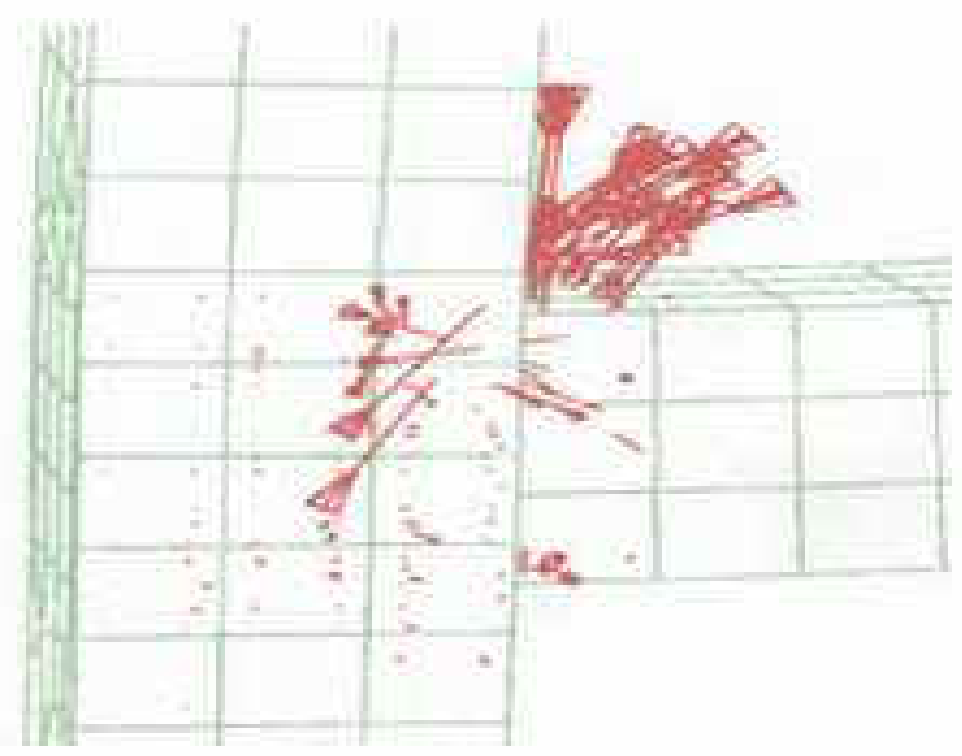

Fig.37.b Deformation Mode by

(JB)-mode (Hitoshi Sh. 2005)

(JB)-mode Joint No.(28)-obtained results

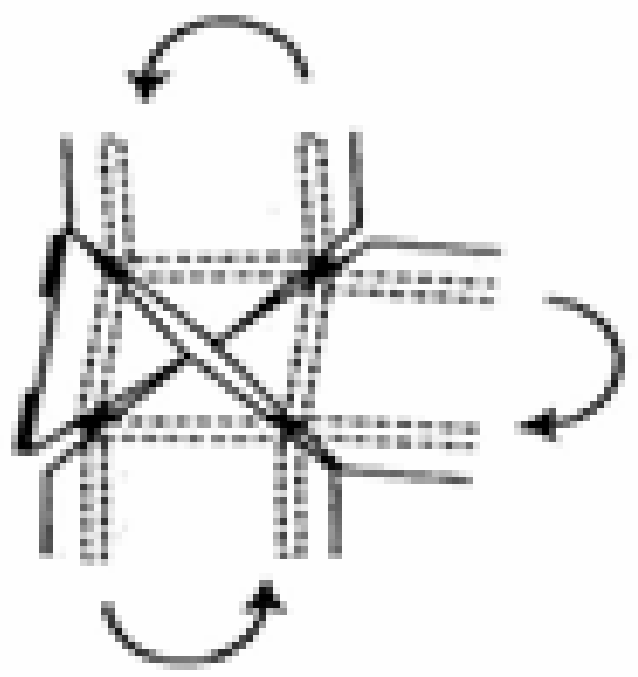

Journal of Engineering Sciences, Assiut University, Faculty of Engineering, Vol. 41, No. 3, May, 2013,E-mail address: jes@aun.edu.eg 
L. K. Idriss et al., Static behaviour of different types of R.C beam-column connections as affected by both value acting lateral horizontal force and grade of used concrete (theoretical study) part two, pp.746 - 814.

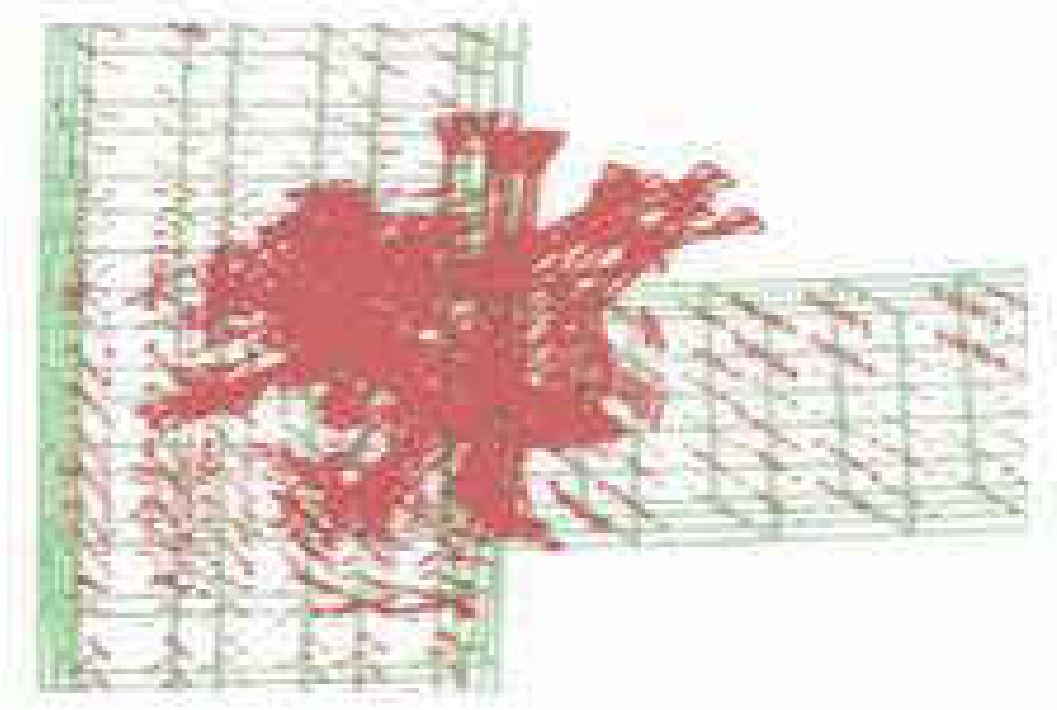

Fig.37.c Deformation Mode by

(J)-mode (Hitoshi Sh. 2005)

(J)-mode Joint No.(32)-obtained results

Fig. 37. Analytical Crack Patterns at Final Loading Stage for Exterior BeamColumn Joint

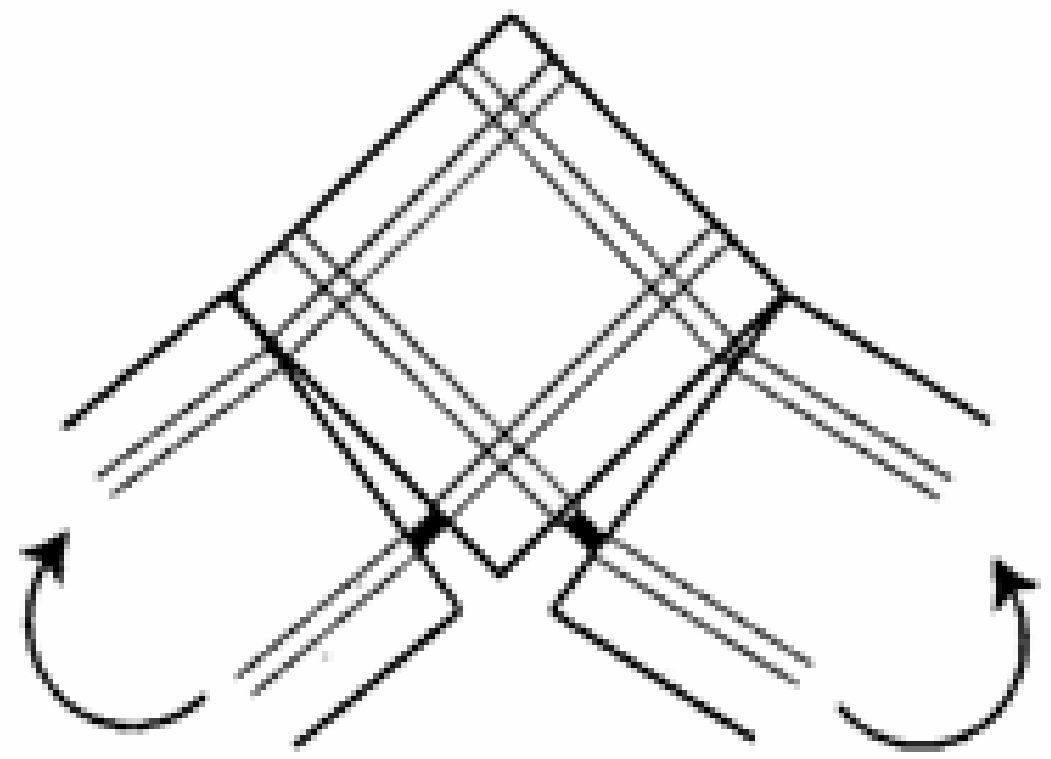

Journal of Engineering Sciences, Assiut University, Faculty of Engineering, Vol. 41, No. 3, May, 2013,E-mail address: jes@aun.edu.eg 
L. K. Idriss et al., Static behaviour of different types of R.C beam-column connections as affected by both value acting lateral horizontal force and grade of used concrete (theoretical study) part two, pp.746 - 814

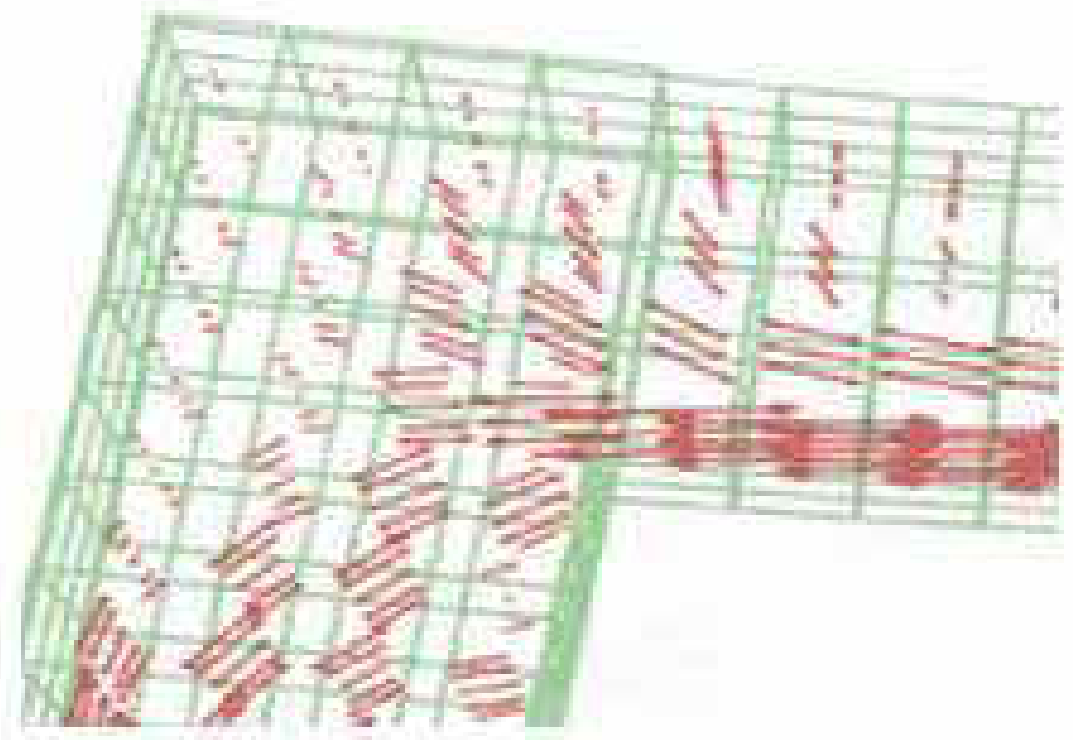

Fig.38.a Deformation Mode by

(B)-mode (Hitoshi Sh. 2010)

(B)-mode Joint No.(36)-obtained results

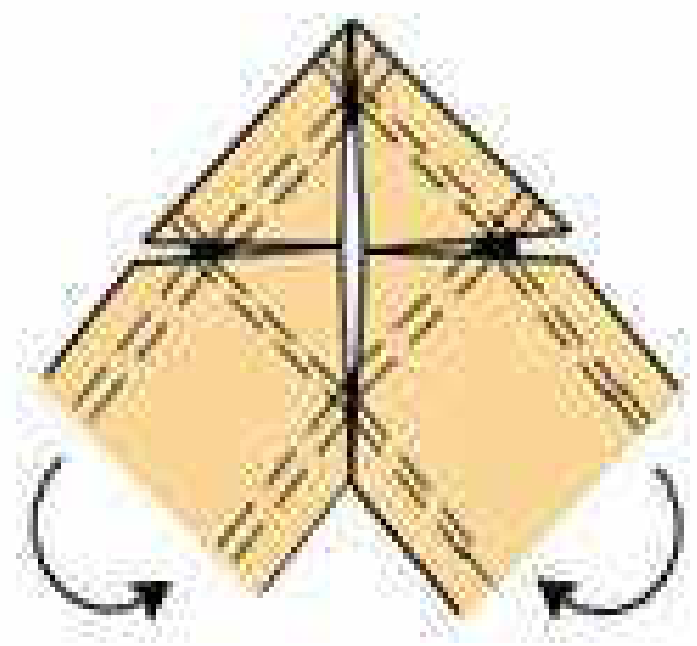

Journal of Engineering Sciences, Assiut University, Faculty of Engineering, Vol. 41, No. 3, May, 2013,E-mail address: jes@aun.edu.eg 
L. K. Idriss et al., Static behaviour of different types of R.C beam-column connections as affected by both value acting lateral horizontal force and grade of used concrete (theoretical study) part two, pp.746 - 814.

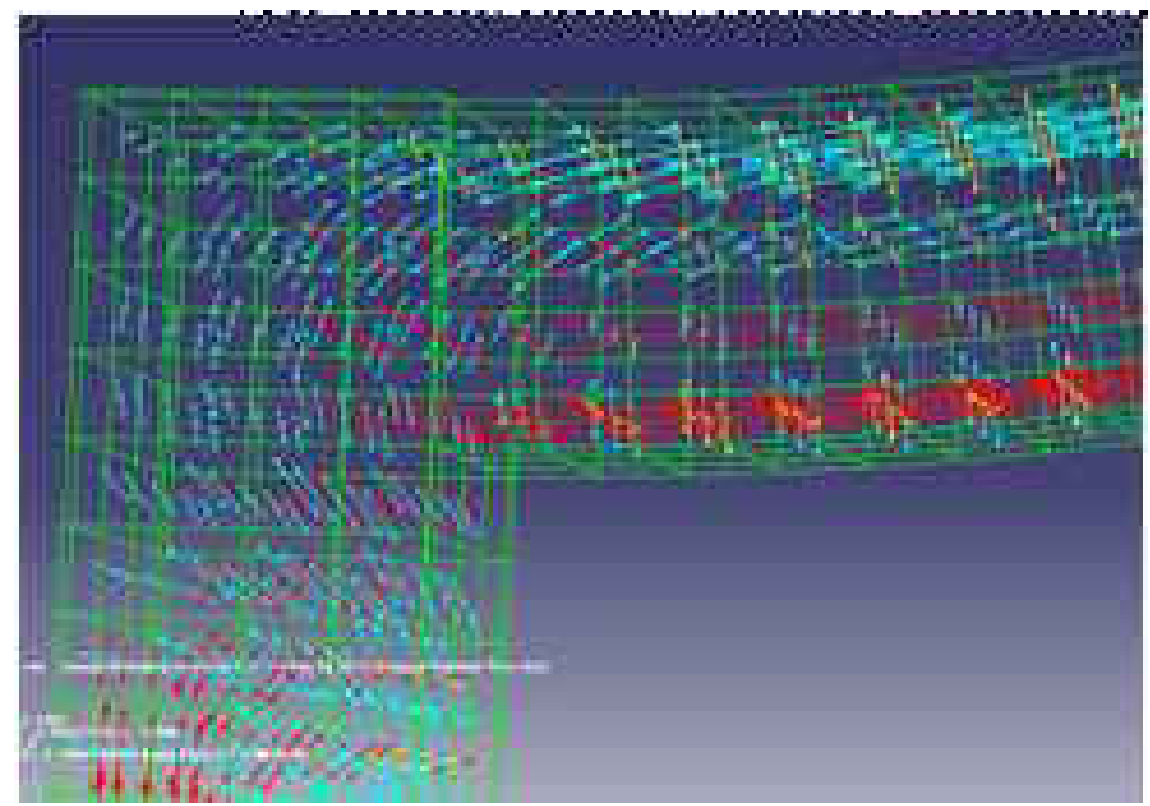

Fig.38.b Deformation Mode by

(JB)-mode (Hitoshi Sh. 2010)

(JB)-mode Joint No.(42)-obtained results

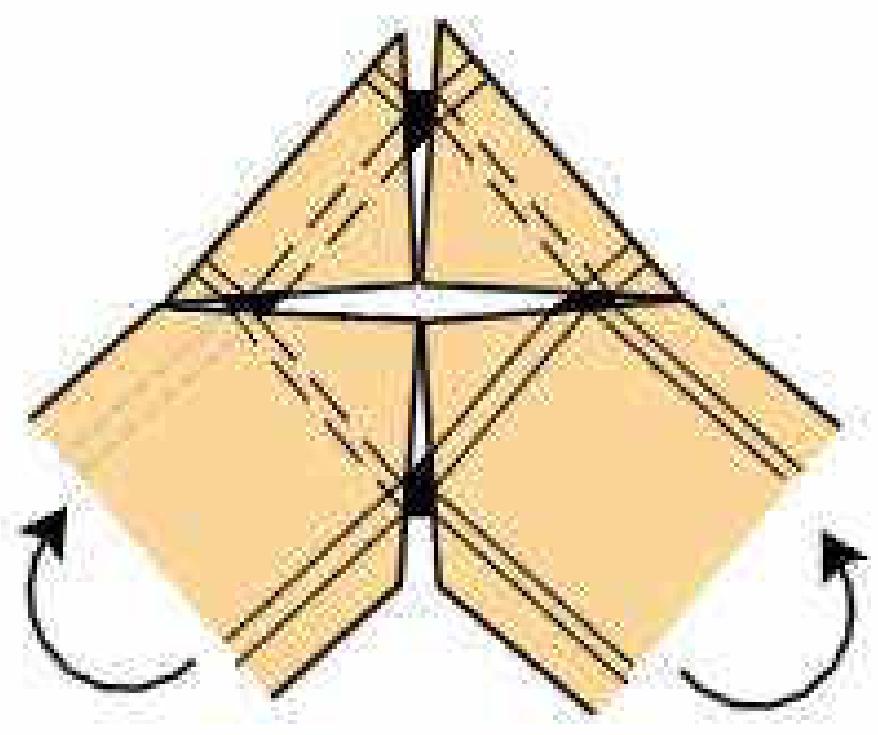

Journal of Engineering Sciences, Assiut University, Faculty of Engineering, Vol. 41, No. 3, May, 2013,E-mail address: jes@aun.edu.eg 
L. K. Idriss et al., Static behaviour of different types of R.C beam-column connections as affected by both value acting lateral horizontal force and grade of used concrete (theoretical study) part two, pp.746 - 814

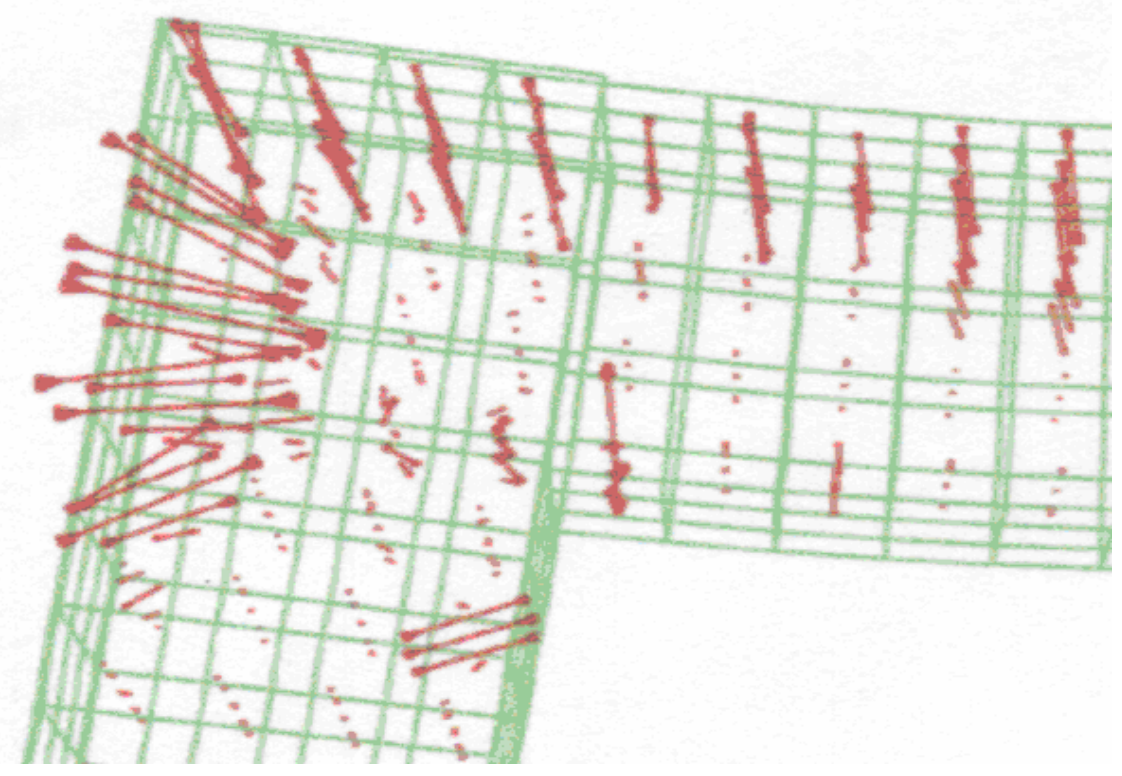

Fig.38.c Deformation Mode by

(J)-mode (Hitoshi Sh. 2010)

(J)-mode Joint No.(48)-obtained results

Fig.38. Analytical Crack Patterns at Final Loading Stage for Corner BeamColumn Joint

\section{Conclusions and Recomendations}

The following conclusions can be drawn out from the performance-based study concerning the parameters affecting the mechanical static behavior of interior, exterior and knee (roof corner) joints, for the case of study of loading.

\subsection{W.R.T stresses point of view:}

\subsubsection{Joint Shear Strength $\left(\tau_{J}\right)$ :}

1. The obtained results on interior, exterior and knee connections indicated that the governing factor influencing the joint shear strength is the concrete compressive strength alone. The concrete strength is considered as the main parameter in determining the ultimate shear capacity.

2. Generally as the compressive strength of concrete increases the corresponding joint shear stress increases too for all types of joint. Also it obvious that the interior joints posses higher joint shear stress in comparison with that for both exterior or corner joint.

Journal of Engineering Sciences, Assiut University, Faculty of Engineering, Vol. 41, No. 3, May, 2013,E-mail address: jes@aun.edu.eg 
L. K. Idriss et al., Static behaviour of different types of R.C beam-column connections as affected by both value acting lateral horizontal force and grade of used concrete (theoretical study) part two, pp.746 - 814 .

3. The $(\mathrm{J})$ mode strength, defined by $\left(\tau_{\mathrm{j}} / f_{\mathrm{c}}^{\prime}\right)$, for the various joints was calculated and plotted against the corresponding compressive strength $\left(f_{\mathrm{c}}^{\prime}\right)$ declared that the $\left(\tau_{\mathrm{j}} / f_{\mathrm{c}}^{\prime}\right)$ mode strength decreases by increasing the grade of concrete. Higher values were corresponding to interior joints rather than that for exterior joints and finally smaller values were for corner joints.

4. Based on our results for the studied joints the following equations 24,25 and 26 are proposed:

$$
\begin{aligned}
& \tau_{j u}=1.56 \times f_{\mathrm{c}}^{0.673} \quad \text { For interior beam-column joint } \\
& \tau_{j u}=1.13 \times \dot{f}_{\mathrm{c}}^{0.573} \quad \text { For exterior beam-column joint } \\
& \tau_{j u}=1.09 \times \dot{f}_{\mathrm{c}}{ }^{0.573} \quad \text { For knee beam-column joint }
\end{aligned}
$$

A comparison of our results for joint shear strength for the studied joints with that given by available codes recommendations declared that :

1. For Interior Joint the maximum shear stress capacity for the studied connections is 69\% lower as per ACI 318M-02 and is lower as 52\% per ECCS 203 (2001), however it is higher than that provided by NZS 3101:1995 per 67\% as well as it is higher than that provided by AIJ 1997 per 52\% and finally it is the same per EN 1998-1:2003.

2. For exterior Joint the maximum shear stress capacity for the studied connections is 45\% lower as per ACI 318M-02 and is lower as 74\% per NZS 3101:1995 however it is higher than that provided by EN 1998-1:2003 per 85\%and, finally it is higher than that provided by AIJ 1997 per $95 \%$.

3. For knee Joint the maximum shear stress capacity for studied connections is $63 \%$ lower as per ACI 318M-02 and higher than that provided by AIJ 1997 per 77\%.

\subsubsection{Joint bond stress $\left(u_{b}\right)$ :}

1. The increase of concrete compressive strength with yielding of the beam reinforcement are effective parameters to increase the bond strength of beam reinforcement over the column width.

2. Based on our results the bond strength can be represented by the equations 27 to 32 for studied joints as follows:

3. For interior joint: The beam bar bond index (BI) was defined by dividing the average bond stress $\left(\mu_{\mathrm{b}}\right)$ by the square root of the concrete and given by Eqs. 27and 28:

$$
\begin{aligned}
& (\mathrm{BI})=\frac{\mathrm{u}_{b}}{\sqrt{\mathrm{f}_{c}^{\prime}}}=\frac{\mathrm{f}_{\mathrm{y}}}{3.5 \sqrt{\mathrm{f}_{c}^{\prime}}} \frac{\mathrm{d}_{b}}{\mathrm{~h}_{c}} \quad \text { for } 2400 \leq f_{\mathrm{y}}<3600 \quad\left(\mathrm{~kg} / \mathrm{cm}^{2}\right) \\
& (\mathrm{BI})=\frac{\mathrm{u}_{b}}{\sqrt{\mathrm{f}_{c}^{\prime}}}=\frac{\mathrm{f}_{\mathrm{y}}}{2 \sqrt{\mathrm{f}_{c}^{\prime}}} \frac{\mathrm{d}_{\mathrm{b}}}{\mathrm{h}_{c}} \quad \text { for } 3600<f_{\mathrm{y}} \leq 4000 \quad\left(\mathrm{~kg} / \mathrm{cm}^{2}\right)
\end{aligned}
$$

where $f_{y}$ : yield strength of beam bars in $\mathrm{kgf} / \mathrm{cm}^{2}, d_{b}$ : diameter of beam bars, $h_{c}$ : column width and $f_{c}$ : concrete compressive strength in $\mathrm{kgf} / \mathrm{cm}^{2}$.

4. For Exterior Joint :the bond index (BI) is given by Eqs. (29) and (30):

Journal of Engineering Sciences, Assiut University, Faculty of Engineering, Vol. 41, No. 3, May, 2013,E-mail address: jes@aun.edu.eg 
L. K. Idriss et al., Static behaviour of different types of R.C beam-column connections as affected by both value acting lateral horizontal force and grade of used concrete (theoretical study) part two, pp.746 - 814

$$
\begin{aligned}
& (\mathrm{BI})=\frac{u_{\mathrm{b}}}{\sqrt{f_{\mathrm{c}}^{\prime}}}=\frac{f_{\mathrm{y}}}{4.50 \sqrt{f_{\mathrm{c}}^{\prime}}} \frac{d_{b}}{h_{\mathrm{c}}} \quad \text { for } 2400 \leq f_{\mathrm{y}}<3600\left(\mathrm{~kg} / \mathrm{cm}^{2}\right) \\
& (\mathrm{BI})=\frac{u_{\mathrm{b}}}{\sqrt{f_{\mathrm{c}}^{\prime}}}=\frac{f_{\mathrm{y}}}{3.20 \sqrt{f_{\mathrm{c}}^{\prime}}} \frac{d_{b}}{h_{\mathrm{c}}} \quad \text { for } 3600<f_{\mathrm{y}} \leq 4000\left(\mathrm{~kg} / \mathrm{cm}^{2}\right)
\end{aligned}
$$

5. For Knee Joint: The bond index (BI) is given by Eqs. (31) and (32):

$$
\begin{aligned}
& (\mathrm{BI})=\frac{u_{\mathrm{b}}}{\sqrt{f_{\mathrm{c}}^{\prime}}}=\frac{f_{y}}{6.20 \sqrt{f_{\mathrm{c}}^{\prime}}} \frac{d_{b}}{h_{c}} \quad \text { for } 2400 \leq f_{y}<3600\left(\mathrm{~kg} / \mathrm{cm}^{2}\right) \\
& (\mathrm{BI})=\frac{u_{\mathrm{b}}}{\sqrt{f_{\mathrm{c}}^{\prime}}}=\frac{f_{y}}{3.20 \sqrt{f_{\mathrm{c}}^{\prime}}} \frac{d_{b}}{h_{c}} \quad \text { for } 3600<f_{\mathrm{y}} \leq 4000\left(\mathrm{~kg} / \mathrm{cm}^{2}\right)
\end{aligned}
$$

6. Finally the value of of the bond index (BI) was suggested for the chosen modeling of bond capacity as follows using the following equation for different types of joints is given by Eqs. (33);

$$
(\mathrm{BI})=\frac{\mathrm{u}_{b}}{\sqrt{\mathrm{f}_{c}^{\prime}}}=k=\text { cons } \tan t
$$

Where $(k)$ is a constant depend on both used grade of concrete as given in the following Table. Suggested Values of (k) for Calculating Average

Bond Capacity of R.C Joints.

\begin{tabular}{|l|c|c|}
\hline \multirow{2}{*}{ Type of Joint } & \multicolumn{2}{|c|}{$(\boldsymbol{k})$ values of $\left(\boldsymbol{f}_{\boldsymbol{c}}^{\prime}\right)$} \\
\cline { 2 - 3 } & $\mathbf{C 2 5 0}$ and C400 & C600 and C1200 \\
\hline Interior joint & 1.80 & 3.20 \\
\hline Exterior joint & 1.58 & 1.70 \\
\hline Knee joint & 1.20 & 1.92 \\
\hline
\end{tabular}

\subsubsection{Axial Principle Stress $(\sigma 1)$ :}

1. The increase of concrete compressive strength is effective to increase the induced principle stresses. The induced principle stresses considerally affected by both grade of concrete and type of joint.

2. Also the ratio of $\left(\sigma_{1} \backslash \dot{f}_{\mathrm{c}}\right)$ usually decreases with the increase of the corresponding concrete compressive strength $\left(f_{c}^{\prime}\right)$. However, interior joints posses higher joint ratio of $\left(\sigma_{1} \backslash \dot{f}_{\mathrm{c}}\right)$ in comparison with that for corner then exterior joint respectively 
L. K. Idriss et al., Static behaviour of different types of R.C beam-column connections as affected by both value acting lateral horizontal force and grade of used concrete (theoretical study) part two, pp.746 - 814.

9.2.W.R.T Deformations and Strains point of view:

\subsubsection{Joint deformation (lateral displacement $\{\delta h\})$ :}

1. Generally as the compressive strength increases the horizontal displacement decreases for the studied joints. Concrete compressive strength has a beneficial effect to decrease horizontal displacement.

2. The interior joint possesses the smallest value of horizontal displacement $\left(\delta_{\mathrm{h}}\right)$ in comparison with that for exterior or corner joint. The corner joint possess the higher value of $\left(\delta_{\mathrm{h}}\right)$.

\subsubsection{Joint deformation (drift ratio angle $\{\theta \%\}$ ):}

1. The increase of concrete grade $\left(f_{c}{ }^{\prime}\right)$ is usually accompanied with a decrease for the induced drift angle $(\theta \%)$ for the studied types of R.C joints. Also the induced drift angle $(\theta \%)$, for a given grade of concrete $\left(f_{\mathrm{c}}{ }^{\prime}\right)$, mainly depends on the type of joint.

2. The interior joint possesses the smallest value of drift angle $(\theta \%)$ in comparison with that for exterior or corner joint. The corner joint possess the higher value of drift angle $(\theta \%)$.

\subsubsection{Joint deformation (shear strain \{distortion $\% \%$ \}):}

1. The increase of concrete grade $\left(f_{\mathrm{c}}{ }^{\prime}\right)$ is usually accompanied with a slight decrease of joint shear distortion $(\gamma \%)$. At the same time the corner joint possess higher value compared with that for both exterior and interior joints. The minimum value of joint shear distortion $(\gamma \%)$ is corresponding to the interior joints.

\subsubsection{Axial principle strains $(\varepsilon 1)$ :}

1. The increase of concrete grade is usually accompanied by an increase in the induced principle strains disregarding the type of joint.

2. Also it is interesting to note that Interior joint usually possess higher values of maximum induced principle strain for any grade of concrete. The smallest values of maximum principle strains are corresponding to corner joints.

3. Increase of concrete grade is usually accompanied by an increase in the induced $\left(\sigma_{1} / \varepsilon_{1}\right)$ ratio. Also its obvious that an interior joints and exterior joint posses the same ratio of increasing the $\left(\sigma_{1} / \varepsilon_{1}\right)$ corresponding to the increasing of concrete grade $\left(f_{\mathrm{c}}{ }^{\prime}\right)$.Also it is indicated that the rate of increase of maximum principle strain with respect to the increase of concrete grade is more or less equal for all kinds of studied joints.

\subsection{W.R.T energy absorption (E.A):}

As the compressive strength of used concrete increases the dissipated energy considerably increases disregarding the type of joint.Mean while for a given concrete grade

Journal of Engineering Sciences, Assiut University, Faculty of Engineering, Vol. 41, No. 3, May, 2013,E-mail address: jes@aun.edu.eg 
L. K. Idriss et al., Static behaviour of different types of R.C beam-column connections as affected by both value acting lateral horizontal force and grade of used concrete (theoretical study) part two, pp.746 - 814

the value of dissipated energy is higher for interior joint rather than that for exterior joint, and at the same time the later one shows a larger value than that for corner joint.

\subsection{W.R.T Mode of Joint Failure:}

Three modes of joint failure were recorded in the case namely:

(B)-mode = beam bond failure : $\mathrm{J}(2,4,6,8)$ for interior joint, $\mathrm{J}(18,20,32)$ Exterior joint and $(34,36)$ for Knee joint.

$(\mathbf{J})$-mode = joint shear failure: $\mathrm{J}(10,12,16)$ for interior joint, $\mathrm{J}(24,26,30)$ Exterior joint and $(34,36)$ for Knee joint.

(JB)-mode = plastic hinge failure: $\mathrm{J}(14)$ for interior joint, $\mathrm{J}(28)$ Exterior joint and $(42,44)$ for Knee joint.

\section{Recommendations}

The following topics can be recommended as subjects for future research study.

1. To modify the joint failure into more ductile beam yield failure mode, it is effective to increase the moment capacity of joint, by increasing of longitudinal reinforcement in joint core inevitably also increase the beam moment capacity relative to the induced moment at beam ends yield in flexure,

2. High strength concrete when used in columns connection needs large quantities of confining reinforcement to ensure ductile behavior, which can be provided by high strength transverse reinforcement. The spacing recommendation for confinement reinforcement from Comittee 352 appears to be valid for high-strength joints so that it is found that the high-strength concrete grades achieved higher levels of ductility, with better energy absorption and an increased initial stiffness in comparison to identically detailed normal strength for internal, external and knee specimens.

3. More experimental tests for RC beam-column connections are needed with specific conditions such as using headed bars or fiber reinforced concrete will be beneficial in the extension of understanding behavior of RC beam-column connections.

4. The suggested joint shear behavior model was constructed based on standard Theoretical tests of RC beam-column connection subassemblies. Because the boundary conditions of RC beam-column connections are often different in real RC moment resisting frames (MRF), the effect of boundary conditions on joint shear behavior could be further investigated

5. Nonlinearity due to concrete spalling and reinforcement buckling has not been taken into account in the present analysis ,hence it is needed further study.

6. More experimental tests is required to take into account the different section geometry of columns beams, it is suggested that circular columns is to may be taken in beam-column connections at the future investigation

Journal of Engineering Sciences, Assiut University, Faculty of Engineering, Vol. 41, No. 3, May, 2013,E-mail address: jes@aun.edu.eg 
L. K. Idriss et al., Static behaviour of different types of R.C beam-column connections as affected by both value acting lateral horizontal force and grade of used concrete (theoretical study) part two, pp.746 - 814.

\section{Notation}

$A_{\mathrm{b}}$

$A_{b s}$

$A_{g}, A_{c}$

$A_{c s}$

$\mathrm{A}_{\mathrm{j}}$

$A_{\text {jh }}$

$\mathrm{A}_{\mathrm{s} 1}, \mathrm{~A}_{\mathrm{s} 2}$

$A_{\text {st }}$

$A_{\mathrm{s}}^{*}$

$b_{b}, b_{c}$

$\mathrm{b}_{\mathrm{j}}$

$\mathrm{d}_{\mathrm{c}}, \mathrm{d}_{\mathrm{b}}$

$D_{j}$

$\left({ }_{d} d_{d}\right)$

E.A

$E_{c}$

Es

$\mathrm{E}_{0}$

$\dot{f}_{\text {c }}, \dot{f}_{\mathrm{c}}, f_{\mathrm{cd}}$

$f_{y}$

$f_{\text {jy }}$

$f_{\text {by }}$

$\mathrm{H}$

$\mathrm{h}_{\mathrm{c}}, \mathrm{h}_{\mathrm{col}}$

$\mathrm{h}_{\mathrm{j}}$

J

$\mathrm{k}$

$L_{b}, L$

$\mathrm{L}_{\mathrm{c}}$

$\mathrm{N}_{\mathrm{c}}$

$V_{b}$

$V_{c}$

$V_{\mathrm{j}}$

$\mathrm{V}_{\mathrm{jh}}$

$\gamma$

$\gamma_{\mathrm{ACI}}$

$\phi$

$\eta$

$\delta_{\mathrm{h}}$
Gross area of cross section of beam

Area of the other beam reinforcement

Gross area of cross section of column

Area of the column reinforcement

Horizontal sectional area of joint core

Total cross-sectional area of horizontal joint transverse reinforcement

Top and bottom reinforcements of beam respectively

Area of longitudinal reinforcement

Greater of the area of top or bottom beam reinforcement passing through the joint

Width of beam, width of column

Effective width of joint

Effective depth of beam, effective depth of column

Effective joint depth

Diameter of longitudinal reinforcing bar

Energy absorption

The concrete static modulus

The steel elastic modulus

The plastic strain

Compressive cylinder strength of concrete

Yield strength of steel reinforcement

Yield stress of horizontal joint transverse reinforcement

Yield stress of longitudinal beam reinforcement

The total height of the columns above and below the joint

Depth of column

Effective depth of joint

Range of mode strength

Averaged bond strength in beam-column joint

Length of the beam right and left the joint

The heights of the columns above and below the joint

Axial column loads

Beam shear force

Column shear force

Joint shear stress

Nominal shear stress

Nominal strength coefficient

Joint shear strength factor

Reduction factor for effect of plane geometry

De notes the reduction factor on concrete compressive strength due to tensile strains in transverse direction

Lateral top displacement

Journal of Engineering Sciences, Assiut University, Faculty of Engineering, Vol. 41, No. 3, May, 2013,E-mail address: jes@aun.edu.eg 
L. K. Idriss et al., Static behaviour of different types of R.C beam-column connections as affected by both value acting lateral horizontal force and grade of used concrete (theoretical study) part two, pp.746 - 814

\begin{tabular}{ll}
\hline$\varepsilon_{1}$ & Principal axial strains \\
$\theta$ & Story drift \% \\
$\mu_{\mathrm{B}}$ & Joint bond stress \\
$\gamma$ & Joint shear strain distortion, \\
$\sigma_{\mathrm{B}}$ & Concrete compressive strength \\
$\sigma_{\mathrm{o}}, \sigma_{\mathrm{col}}$ & Column axial stress \\
$\sigma_{\mathrm{c}}$ & Concrete stress block \\
$\tau_{\mathrm{J}}, \tau_{j u}$ & Joint shear stress \\
$\sigma_{1}$ & principle axial stress \\
$\tau_{\mathrm{J}}$ & Joint shear stress \\
$\rho_{w}$ & Joint shear reinforcement
\end{tabular}

\section{References}

[1]ABQUS, ABAQUS Theory Manual, (v6.7-1) (2002), Training Manual, (v6.5-1) (2004), User Manual, (v6.5-1) (2000).

[2] ACI 318M-02 (2002): "Building code requirements for structural concrete and commentary", Reported by ACI Committee 318, American Concrete Institute, Farmington Hills, Michigan.

[3] ACI-ASCE Committee 352 (1995): "Recommendations for Design of Beam-Column Joints in Monolithic Reinforced Concrete Structures" (AC1 352R-95), American Concrete Institute, Detroit, Michigan, 1995.

[4] Ahmed Hassan Aly Abdel- Kreem (2003): "Enhancement Of Beam - Column Connection Under Seismic Loads Recommendations for design of beam-column joints in monolithic reinforced concrete structures ”, Benha Higher Institute of Technology ,May (2003).

[5] Bing Li, Yiming Wu, and Tso- Chien Pan (2003): "Seismic Behavior of Non seismically Detailed interior Beam-Wide Column joints-Part 2: Theoretical Comparisons and Analytical Studies" ACI Structural journal, V.100, No.1, January-February. 2003. P.56-65.

[6] Eligehausen, R., Popov, E.P. and Bertero, V.V. (1993): "Local Bond Stress-Slip Relationships of Deformed Bars under Generalized Excitations." EERC Report 83/23. Berkeley: University of California.

[7] EN 1998-1:2003: "General rules-specific rules for various materials and elements", Euro code 8: Design Provisions for Earthquake Resistant Structures.

[8] Felicia Thien Ying Chik (2007): "Influence of Different Concrete Strength on the Behavior of Interior Reinforced Concrete Beam-Column Joint” Degree of master of civil Engineering University of Technology Malaysia, November 2007.

[9] Hitoshi Shiohara, Afonso Toshiiti Sato, Shunsuke Otani and Taizo Matsumori (2002): "Effects of Beam Pre stressing Force on the Strength and Failure Mode of R/C Exterior Beam-Column Joints." The University of Tokyo, Graduate School of Engineering, Department of Architecture, Research Associate 2002 Fib.

[10] Hitoshi Shioharal (2005): New Model for Joint Shear Failure of R/C Exterior Beam-Column Joints". Associate Professor, Department of Architecture, Graduate School of Engineering, The University of Tokyo, Tokyo, 113-8656 Japan.

[11] Hitoshi S. HIOHARA (2004): "Quadruple Flexural Resistance in R/C Beam-Column Joints" $13^{\text {th }}$ World Conference on Earthquake Engineering Vancouver, B.C., Canada August 1-6, 2004 Paper No. 491.

[12] J. Lee and G. L. Fenves (1998): "Plastic-Damaged model for cycling loading of concrete structures", 124(8), Journal of Engineering Mechanics.

Journal of Engineering Sciences, Assiut University, Faculty of Engineering, Vol. 41, No. 3, May, 2013,E-mail address: jes@aun.edu.eg 
L. K. Idriss et al., Static behaviour of different types of R.C beam-column connections as affected by both value acting lateral horizontal force and grade of used concrete (theoretical study) part two, pp.746 - 814.

[13] Jaehong Kim and James M. La Fave (2008): "Joint Shear Behavior Prediction in RC BeamColumn Connections Subjected to Seismic Lateral Loading". Structural Consultant, S.K Associate Professor, Dept. of Civil and Environmental Engineering, University of Illinois at Urbana-Champaign, Urbana, IL, U.S.A. October 12-17, 2008, Beijing, China.

[14] Josef Hegger, Alaa sheriff, and Wolfgang Roeser (2003): "Non seismic Design of beamColumn Joints" ACI Structural journal, V.100, No.5, September-October. 2003. P. 654-658.

[15] John S. Stehle, Helen Goldsworthy, and Priyan Mendis (2001): "Reinforced Concrete interior Wide-Band Beam-column Connections Subjected to lateral Earthquake Loading" ACI Structural journal/May-June 2001.

[16] Kazuhiro Kitayama, Shunsuke Otani and Hiroyuki Aoyama (1991): "Development of Design Criteria for RC Interior Beam-Column Joints.” ACI SP-123 Design of Beam-Column Joints for Seismic Resistance, James O. Jersey Editor, American Concrete Institute, Michigan, 1991, pp. 97-123.

[17] Kumar, S.R.S. B.V. and G.S.B., (2002): "Hysteretic behavior of lightly reinforced - concrete exterior beam-to-column joint sub-assemblages". J. Struc. Eng. SERC, 29 (1): 31-37.

[18] Kupfer, H., Hilsdorf, H.K., Rüsch, H. (2011): Behavior of Concrete under Biaxial Stress, Journal ACI, Proc. V.66, No.8, Aug., pp. 656-666.

[19] Laura N. Lowes Nilanjan Mitra and Arash Altoontash, (2004): “A Beam-Column Joint Model for Simulating the Earthquake Response of Reinforced Concrete Frames", Pacific Earthquake Engineering Research Center College of Engineering University of California, Berkeley February 2004.

[20] Laura Nicole Lowes (1992): "Finite Element Modeling of Reinforced Concrete Beam-Column Bridge Connections" B.S (University of Washington 1992 dissertation book).

[21] Lowes, L.N. (2002): "Finite Element Modeling of Reinforced Concrete Beam-Column Bridge Connections". Dissertation University of California, Berkeley.

[22] Masaru Teraoka, Kazuya Hayashi Satoshi Sasaki, and Naoki Takamori (2005): "Estimation of restoring force characteristics in the interior beam and-Column Sub assemblages of R/C Frames" Fujita Technical Research Report No.41 2005.

[23] NZS 3101 (1995): “The design of concrete structures", Concrete Structures Standard, Part 1: Code of Practice, Part 2: Commentary, Standards New Zealand, Wellington, New Zealand, $256 \mathrm{pp} \& 264 \mathrm{pp}$.

[24] S. R. Uma AND Sudhir K. Jain (2006): "Seismic design of beam-column joints in RC moment resisting frames" - Review of codes Structural Engineering and Mechanics, Vol. 23, No. 5 (2006) 579-597 579NDepartment of Civil Engineering, Indian Institute of Technology, University of Canterbury, New Zealand.

[25] Sam Lee (2008): "Nonlinear Dynamic Earthquake Analysis of Skyscrapers" Guangzhou Scientific Computing Consultants Co. Ltd, 507/140 Dungeon Xi Rd, Guangzhou 510170, 7 China, szslee@gmail.com CTBUH 8th World Congress, Dubai, 3-5 March 2008.

[26] Shima, H., Chou, L.L. and Okamura, H. (1997): "Bond Characteristics in Post-Yield Range of Deformed Bars." Concrete Library of JSCE 10: 113-124.

[27] Teeraphot Supaviriyakita, Amorn Pimanmasa and Pennung Warnitchai, (2008): "Nonlinear Finite Element Analysis of Non-Seismically Detailed Interior Reinforced concrete Beam-Column Connection under Reversed Cyclic Load".

Journal of Engineering Sciences, Assiut University, Faculty of Engineering, Vol. 41, No. 3, May, 2013,E-mail address: jes@aun.edu.eg 
L. K. Idriss et al., Static behaviour of different types of R.C beam-column connections as affected by both value acting lateral horizontal force and grade of used concrete (theoretical study) part two, pp.746 - 814

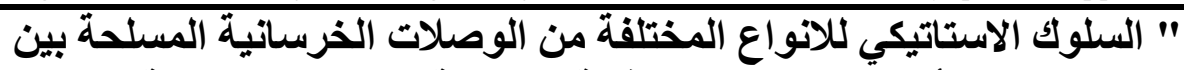

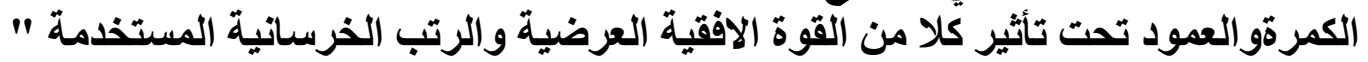 دراسة نظرية}

الملخص:

تم في هذا البحث عمل دراسة نظرية لمجموعة نماذج مقترحة ثلاثية الأبعاد من مناطق إتصال الكمرات بالأعمدة الداخلية و الخارجية والركنية باستخدام برنامج كمبيونر للتحليل الإنشائي 6.7

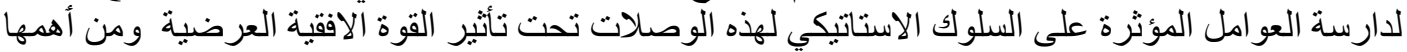

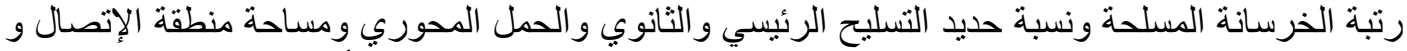

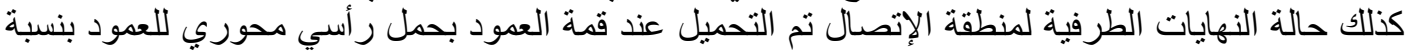

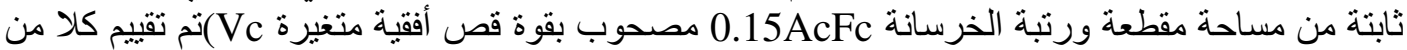

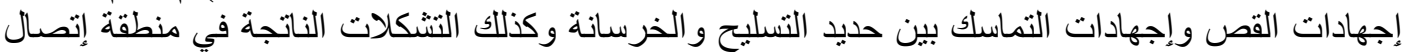

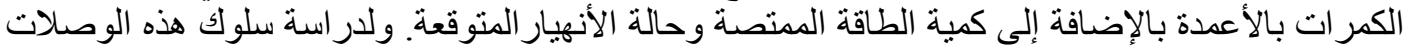

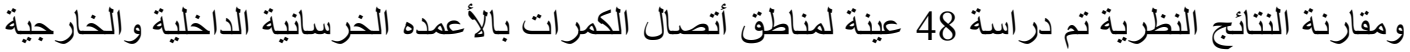
و الركنية حيث تم تثبيت البيانات التالية:

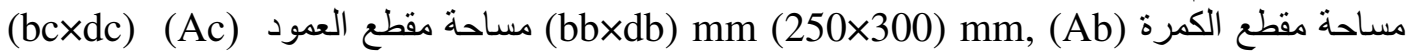
(H×L) m (2.0×3.0) m, (Aj) مساحة منطقة الاتصال الكمرة بالعمود (300×300mm)

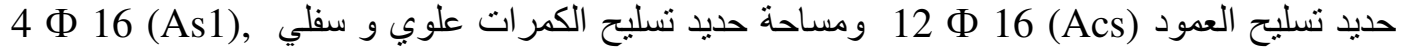

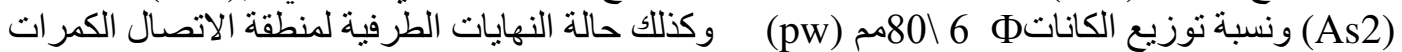

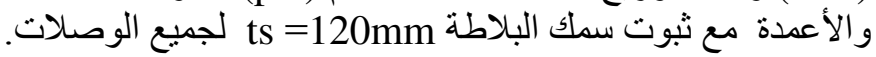
ذللك لرتب الخرسانة المسلحة المختلفة حيث نتر اوح رتب و بالتالي اجهادات الضغط للخرسانة المسلحة بين للمكعبات القياسية بعد 28 يوم و أيضاً رتب حديد التسليح (fc'), of 250-400-600-1200 kglcm2,

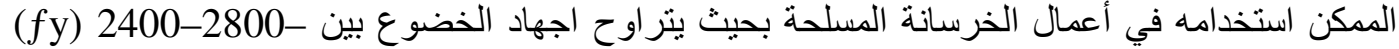

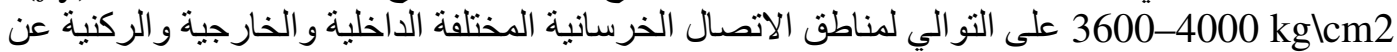

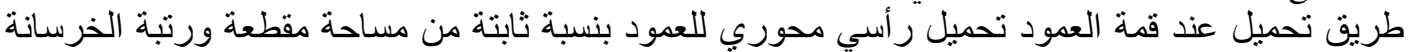
0.15AcFc بالاضافة الي قوة قص أفقية متغيرة (Vc) تنتج منها مجموعة من الاز احات الخطية المتغيرة

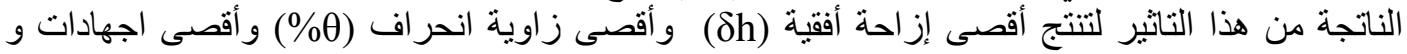

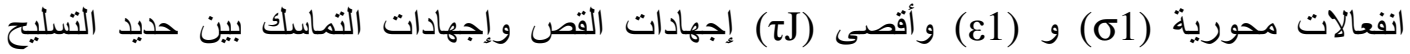

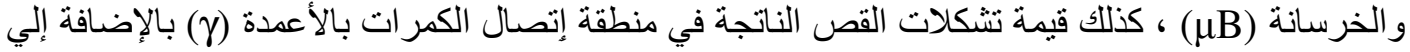
تقدير كمية الطاقة الممتصة ، (E.A) في الوصلة وحالة الانهيار المتوقعة وذللك باستخدام برنامج للتحليل

الاستاتيكي الغير خطي 6.7 ABAQUSICAE version وذلك باستخدام نموذج تم التاكد منه بو اسطة حيث تم دراسة سمات حرجة منتوعة على سلوك منطقة الاتصال بين (الأعمدة و الكمر ات) الخرسانية والتحليل المرن اللدن لجميع الهياكل الخرسانية المسلحة و اختبار السعة القصبة لاحية لاحمال

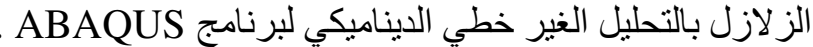

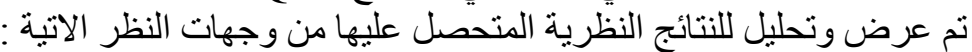

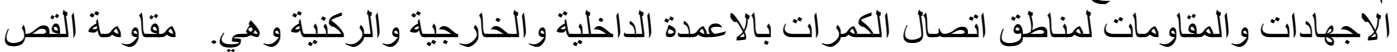

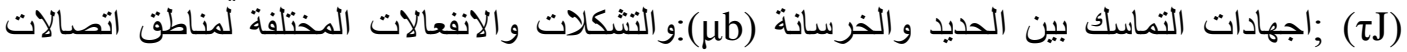

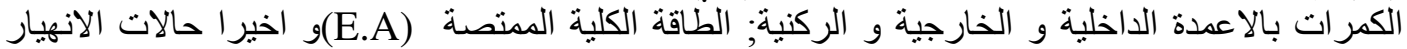

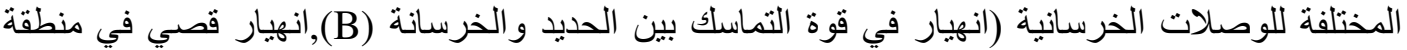

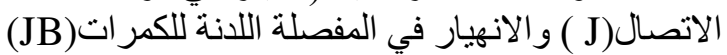

Journal of Engineering Sciences, Assiut University, Faculty of Engineering, Vol. 41, No. 3, May, 2013,E-mail address: jes@aun.edu.eg 
L. K. Idriss et al., Static behaviour of different types of R.C beam-column connections as affected by both value acting lateral horizontal force and grade of used concrete (theoretical study) part two, pp.746 - 814.

التوصيات:

1-لزيادة قدرة و سعة تحمل الوصلات و لتطوير انهيار مناطق الاتصال الكمرات بالأعمدة بأن نكون أكثر

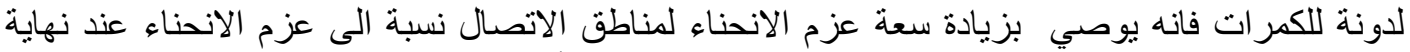

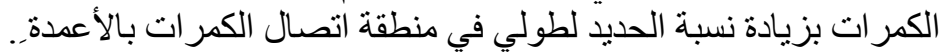

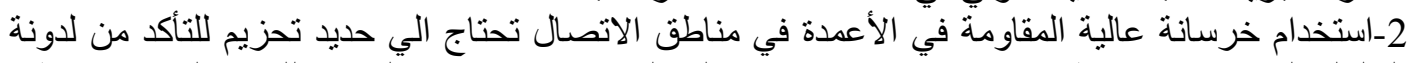

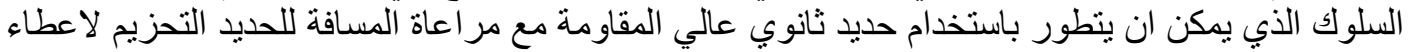

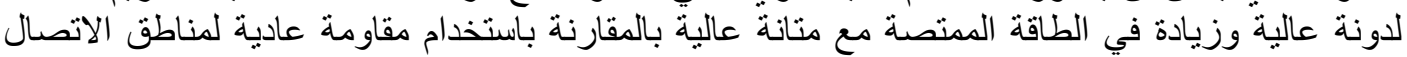
الداخلية و الخارجية و الرية ونية. 3- بعض الدر اسات مطلوبة لمناطق الاتصال الخرسانة المسلحة الكمر ات بالأعمدة باستخدام حديد اضافي

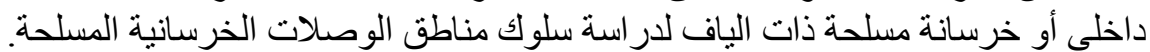

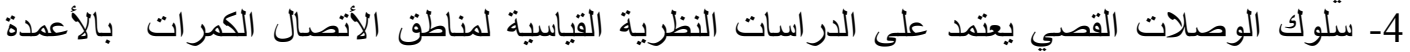

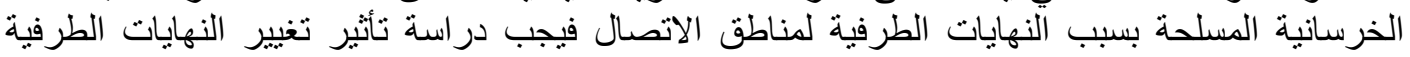
ل لمناطق الاتصال. 5-الاخذ في الاعتبار التحليل الغير خطي عند تقشير الخرسانة وحدوث انحناءحيث ان ذلك غير مأخوذ في

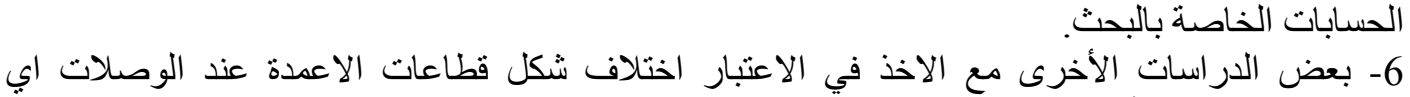

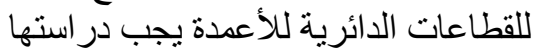

